\title{
Noncommutative symmetric functions III: Deformations of Cauchy and convolution algebras
}

\author{
Gérard Duchamp ${ }^{1}$, Alexander Klyachko², Daniel Krob ${ }^{3}$ \\ and Jean-Yves Thibon ${ }^{4}$ \\ ${ }^{1}$ LIR, Université de Rouen, 76134 Mont Saint-Aignan Cedex, France \\ E-Mail: gedelitp.ibp. fr \\ ${ }^{2}$ Bilkent University, Ankara, Turkey \\ E-Mail: klyachkoefen.bilkent.edu.tr \\ ${ }^{3}$ LITP (IBP, CNRS), Université Paris 7, 2, place Jussieu, 75251 Paris Cedex 05, France \\ E-Mail: dkelitp.ibp.fr \\ ${ }^{4}$ IGM, Université de Marne-la-Vallée, 2, rue de la Butte-Verte, 93166 Noisy-le-Grand Cedex, France \\ E-Mail: jyteuniv-mlv.fr
}

\begin{abstract}
This paper discusses various deformations of free associative algebras and of their convolution algebras. Our main examples are deformations of noncommutative symmetric functions related to families of idempotents in descent algebras, and a simple $q$-analogue of the shuffle product, which has unexpected connections with quantum groups, hyperplane arrangements, and certain questions in mathematical physics (the quon algebra, generalized Brownian motion).
\end{abstract}

Keywords: Symmetric functions, Descent algebras, Free Lie algebras, Quantum shuffle

\section{Introduction}

This article is devoted to the investigation of certain deformations of free associative (or tensor) algebras and of their convolution algebras. Typically, the deformations we are interested in depend on one or several parameters and are trivial in the sense of the deformation theory of algebras. That is, for generic values of these parameters there exists a conjugating isomorphism

$$
u \odot v=f\left(f^{-1}(u) f^{-1}(v)\right)
$$

between the deformed product $\odot$ and the original one. However, for specific values of the parameters, the deformed product degenerates in a non-trivial way, a situation which allows for the representation of complicated algebras as limiting cases of well-understood ones.

1365-8050 (C) 1997 Chapman \& Hall 
The motivation for this investigation was provided by examples of direct sum decompositions of the free associative algebra $K\langle A\rangle$, regarded as the universal enveloping algebra of the free Lie algebra $L(A)$

$$
K\langle A\rangle=\bigoplus_{\lambda} U_{\lambda}
$$

analogous to the Poincaré-Birkhoff-Witt decomposition, i.e. $\lambda$ runs through the set of all partitions, $U_{0}=K$ and $U_{1}=L(A)$.

In these examples, each module $U_{\lambda}$ is the image of the homogeneous component $K\langle A\rangle_{n}$ of degree $n$ of $K\langle A\rangle$ by a certain idempotent $e_{\lambda}$ of the group algebra of the symmetric group $K\left[\mathfrak{S}_{n}\right]$, acting on the right by $\left(x_{1} x_{2} \cdots x_{n}\right) \cdot \sigma=x_{\sigma(1)} x_{\sigma(2)} \cdots x_{\sigma(n)}$ (where $x_{i} \in A$ ).

In the case of the Poincaré-Birkhoff-Witt decomposition, coming from the identification of $K\langle A\rangle$ with the symmetric algebra $S(L(A)), U_{\lambda}$ is the subspace spanned by symmetrized products of Lie polynomials

$$
\left(P_{1}, P_{2}, \ldots, P_{r}\right)=\frac{1}{r !} \sum_{\sigma \in \mathfrak{S}_{r}} P_{\sigma(1)} P_{\sigma(2)} \cdots P_{\sigma(r)}
$$

such that each $P_{i}$ is homogeneous of degree $\lambda_{i}$. The corresponding idempotents, introduced by Garsia and Reutenauer [1], are refinements of the so called Eulerian idempotents (cf. Reutenauer [2]), which arise, for example, in the computation of the Hausdorff series [3], or in the study of the Hochschild cohomology of commutative algebras $[4,5]$.

The Garsia-Reutenauer idempotents $e_{\lambda}$ form, taking all partitions of a given $n$, a complete set of orthogonal idempotents of a remarkable subalgebra $\Sigma_{n}$ of $K\left[\boldsymbol{S}_{n}\right]$, discovered by Solomon [6] and called the descent algebra. It has been shown [7] that such complete sets can be constructed for all descent algebras from any sequence $\left(e_{n}\right)$ of Lie idempotents of $\Sigma_{n}$, i.e. idempotents projecting $K\langle A\rangle_{n}$ onto $L_{n}(A)$. In particular, using the deformation theory of noncommutative symmetric functions, one can obtain interesting sequences of Lie idempotents, depending on one or more parameters, and interpolating in a natural way between all known examples $[8,7]$. This leads to various deformations of the GarsiaReutenauer idempotents and of the Eulerian idempotents, and the first question is certainly to explicit the modules $U_{\lambda}$ onto which they project. The deformation technique presented in Sect. 3 provides the following answer (Sect. 7, Prop. 7.4):

There exists for each $n$ a vector $p=\left(p_{I}\right)$ indexed by compositions of $n$, satisfying $\sum_{I} p_{I}=$ 1 , such that $U_{\lambda}$ is spanned by the weighted symmetrized products

$$
\left(P_{1}, P_{2}, \ldots, P_{r}\right)_{p}=\sum_{\sigma \in \mathcal{G}_{r}} p_{\lambda \cdot \sigma} P_{\sigma(1)} P_{\sigma(2)} \cdots P_{\sigma(r)}
$$

where $\lambda=\left(\lambda_{1}, \ldots, \lambda_{r}\right)$ and each $P_{i} \in L_{\lambda_{i}}(A)$.

The weights $p_{I}$ are explicited for several interesting examples.

The only recorded example of decomposition (1) which does not come from a sequence of Lie idempotents in descent algebras is the so-called orthogonal decomposition (cf. Duchamp [9]). It has been shown by Ree [10] that if one endows $K\langle A\rangle$ with the scalar product for which words form an othonormal basis, the orthogonal complement of $L(A)$ is the space spanned by proper shuffles $u \amalg v, u, v \neq 1$. The orthogonal Lie idempotent $\pi_{n}$ is the orthogonal projector from $K\langle A\rangle_{n}$ onto $L_{n}(A)$. This idempotent is not in 
the descent algebra, and it would be of interest to understand its structure. The orthogonal decomposition of the $K\langle A\rangle$ can be refined into a decomposition of type (1), where $U_{\lambda}$ is now spanned by shuffles of homogeneous Lie elements

$$
P_{1} \uplus P_{2} \amalg \cdots \uplus P_{r}
$$

with each $P_{i}$ of degree $\lambda_{i}$. The relationship between the projectors $\pi_{n}=e_{(n)}$ of this decomposition and the other projectors $e_{\lambda}$ is somewhat analogous to that encountered in the case of the descent algebra, but considerably more intricate.

To understand this analogy, we were led to introduce a $q$-analogue of the shuffle product, which strictly speaking, is rather a deformation of the concatenation product (obtained for $q=0$ ), recursively defined by

$$
a u \odot_{q} b v=a\left(u \odot_{q} b v\right)+q^{|a u|} b\left(a u \odot_{q} v\right)
$$

where $a, b \in A$ and $u, v \in A^{*}$. This product degenerates at roots of unity, and in particular gives the standard (commutative) shuffle product for $q=1$. We conjecture that its convolution algebra degenerates for $q \rightarrow 1$ into a commutative algebra which is associated with a Ree type decomposition

$$
K\langle A\rangle=K \oplus L \oplus L \amalg L \oplus \cdots
$$

where $L$ is a subspace which has the same Hilbert series as the free Lie algebra (this subspace can be explicited). A challenging problem would be to find a good deformation of the shuffle product giving the convolution algebra relevant to the case of the orthogonal idempotent as a degenerate case.

It turns out that the $q$-shuffle, as well as the elements $U_{n}(q)=\sum_{\sigma \in \mathfrak{S}_{n}} q^{\ell(\sigma)} \sigma$, which are naturally associated with it, have already occured in the literature in several apparently unrelated contexts.

First, the $q$-shuffle algebra is the simplest non-trivial case of a very general construction due to Rosso, obtained in the context of the theory of quantum groups. Moreover, the $q$-shuffle algebra is isomorphic to the free associative algebra iff $U_{n}(q)$ is invertible for all $n$. The computation of the determinant of $U_{n}(q)$ (regarded as an operator of the regular representation of $\mathfrak{S}_{n}$ ) already occured in a problem of physics (the Hilbert space representability of the quon algebra, describing hypothetical particles violating Bose or Fermi statistics [11]), and was solved by Zagier, who also computed $U_{n}(q)^{-1}$ by means of certain factorization formulas. The same problem was also solved independently by Bożejko and Speicher [12] who encountered it in the investigation of a generalization of Brownian motion. Surprisingly enough, Zagier's formula for $\operatorname{det} U_{n}(q)$ turns out to be a special case of a recent formula of Varchenko [13], giving the determinant of what he calls the quantum bilinear form of a hyperplane arrangement. To complete the picture, we mention that the $q$-shuffle also has a natural interpretation whithin the representation theory of the 0-Hecke algebras of type $A$ [15]. These aspects of the $q$-shuffle are reviewed, and the various connections are exploited in order to give generalizations or simplifications of known results when possible. For example, we will see that one can construct a quantum shuffle from any solution of the Yang-Baxter equation (without spectral parameters), and that the Hall-Littlewood symmetric functions or the $q$-Fock spaces of Kashiwara, Miwa and Stern [16] zan be regarded as examples of this construction. Also, we generalize Zagier's factorizations to identities in the algebra of the infinite hraid groun, and give some applications (some similar results were obtained independently by Meljanac and Svrtan [17]).

This paper is structured as follows. We first recall the basic definitions concerning noncommutative symmetric functions [18], which provide the convenient formalism for computing in convolution algebras 
(Sect. 2). Next, we present a general deformation pattern and give some simple properties (Sect. 3). In Sect. 4 we introduce the $q$-shuffle and derive its fundamental properties. We review the quon algebra, the work of Zagier, and give some details on the interpretation in terms of the 0-Hecke algebra. In Sect. 5 , we study the $q$-shuffle algebra as a Hopf algebra, and present our conjecture concerning the limit $q \rightarrow 1$ of its convolution algebra. In Sect. 6, we discuss Rosso's quantum shuffles and exhibit some new examples. Next, we generalize to the braid group some of the formulas which occured in the study of the $q$-shuffle, explain the connection with Varchenko's construction, and illustrate the general results on an example constructed from the standard Hecke-type solution of the Yang-Baxter equation. Finally, Sect. 7 is devoted to the description of the decompositions of the free associative algebra obtained from deformations of the Garsia-Reutenauer idempotents.

\section{Noncommutative Symmetric Functions}

\subsection{Definitions}

The algebra of noncommutative symmetric functions, defined in Gelfand et al. [18]. is the free associative algebra $\mathbf{S y m}=\mathbf{Q}\left\langle S_{1}, S_{2}, \ldots\right\rangle$ generated by an infinite sequence of noncommutative indeterminates $S_{k}$, called the complete symmetric functions. We set for convenience $S_{0}=1$. Let $t$ be another variable commuting with all the $S_{k}$. Introducing the generating series

$$
\sigma(t):=\sum_{k=0}^{\infty} S_{k} t^{k}
$$

one defines other families of noncommutative symmetric functions by the following relations:

$$
\begin{gathered}
\lambda(t)=\sigma(-t)^{-1} \\
\frac{d}{d t} \sigma(t)=\sigma(t) \psi(t), \quad \sigma(t)=\exp (\Phi(t))
\end{gathered}
$$

where $\lambda(t), \psi(t)$ and $\phi(t)$ are the generating series

$$
\begin{gathered}
\lambda(t):=\sum_{k=0}^{\infty} \Lambda_{k} t^{k} \\
\psi(t):=\sum_{k=1}^{\infty} \Psi_{k} t^{k-1}, \quad \Phi(t):=\sum_{k=1}^{\infty} \frac{\Phi_{k}}{k} t^{k}
\end{gathered}
$$

The noncommutative symmetric functions $\Lambda_{k}$ are called elementary functions, and $\Psi_{k}$ and $\Phi_{k}$ are respectively called power sums of first and second kind.

The algebra $\mathbf{S y m}$ is graded by the weight function $w$ defined by $w\left(S_{k}\right)=k$. Its homogeneous component of weight $n$ is denoted by $\mathbf{S y m}_{n}$. If $\left(F_{n}\right)$ is a sequence of noncommutative symmetric functions with $F_{n} \in \mathbf{S y m}_{n}$ for $n \geq 1$, we set for a composition $I=\left(i_{1}, \ldots, i_{r}\right)$

$$
F^{I}=F_{i_{1}} F_{i_{2}} \ldots F_{i_{r}}
$$

The families $\left(S^{I}\right),\left(\Lambda^{I}\right),\left(\Psi^{I}\right)$ and $\left(\Phi^{I}\right)$ are homogeneous bases of Sym. 
The algebra Sym can also be endowed with a Hopf algebra structure. Its coproduct $\Delta$ is defined by any of the following equivalent formulas:

$$
\begin{aligned}
\Delta\left(S_{n}\right) & =\sum_{k=0}^{n} S_{k} \otimes S_{n-k} & \Delta\left(\Lambda_{n}\right) & =\sum_{k=0}^{n} \Lambda_{k} \otimes \Lambda_{n-k} \\
\Delta\left(\Psi_{n}\right) & =1 \otimes \Psi_{n}+\Psi_{n} \otimes 1 & \Delta\left(\Phi_{n}\right) & =1 \otimes \Phi_{n}+\Phi_{n} \otimes 1
\end{aligned}
$$

The free Lie algebra $\mathcal{L}=L(\Phi)$ generated by the family $\left(\Phi_{n}\right)_{n \geq 1}$ is then the Lie algebra of primitive elements for $\Delta$.

The set of all compositions of a given integer $n$ is equipped with the reverse refinement order, denoted $\preceq$. For instance, the compositions $J$ of 4 such that $J \preceq(1,2,1)$ are exactly $(1,2,1),(3,1),(1,3)$ and (4). The ribbon Schur functions $\left(R_{I}\right)$, originally defined in terms of quasi-determinants (cf. Gelfand and Retakh $[19,20])$, can also be defined by one of the two equivalent relations:

$$
S^{I}=\sum_{J \preceq I} R_{I} \quad R_{I}=\sum_{J \preceq I}(-1)^{\ell(I)-\ell(J)} S^{J}
$$

where $\ell(I)$ denotes the length of the composition $I$. One can easily check that the family $\left(R_{I}\right)$ is a homogenous basis of Sym.

The commutative image of a noncommutative symmetric function $F$ is the (commutative) symmetric function $f$ obtained by applying to $F$ the algebra morphism which maps $S_{n}$ onto $h_{n}$ (using here the notations of Macdonald [21]). The commutative image of $\Lambda_{n}$ is $e_{n}$ and the power sums $\Psi_{n}$ and $\Phi_{n}$ are both mappeu $\mathbf{t u} \mu_{n}$. Finälily, $R_{I}$ is sent to an ordinary ribbon Schur function, which will be denoted by $r_{I}$.

\subsection{Relations with Solomon's Descent Algebra}

There is a noncommutative analog of the well known correspondence between symmetric functions and characters of symmetric groups, where the character ring of a symmetric group is replaced by the descent algebra, in the sense of Solomon [6]. Recall that an integer $i \in[1, n-1]$ is said to be a descent of a permutation $\sigma \in \mathfrak{S}_{n}$ iff $\sigma(i)>\sigma(i+1)$. The descent set of a permutation $\sigma \in \boldsymbol{S}_{n}$ is the subset of $[1, n-1]$ consisting of all descents of $\sigma$. If $I=\left(i_{1}, \ldots, i_{r}\right)$ is a composition of $n$, one associates with it the subset $D(I)=\left\{d_{1}, \ldots, d_{r-1}\right\}$ of $[1, n-1]$ defined by $d_{k}=i_{1}+\cdots+i_{k}$. Let $D_{I}$ be the sum in $\mathbf{Z}\left[\mathfrak{S}_{n}\right]$ of all permutations with descent set $D(I)$. Solomon showed that the $D_{I}$ form a basis of a subalgebra of $\mathbf{Z}\left[\mathfrak{S}_{n}\right]$ which is called the descent algebra of $\mathfrak{S}_{n}$ and denoted by $\Sigma_{n}$ [6]. One can define an isomorphism of graded vector spaces:

$$
\alpha: \mathbf{S y m}=\bigoplus_{n=0}^{\infty} \mathbf{S y m}_{n} \longrightarrow \Sigma=\bigoplus_{n=0}^{\infty} \Sigma_{n}
$$

by

$$
\alpha\left(R_{I}\right)=D_{I}
$$

The direct sum $\Sigma$ can be endowed with an algebra structure by extending the natural product of its components $\Sigma_{n}$, setting $x y=0$ for $x \in \Sigma_{p}$ and $y \in \Sigma_{q}$ when $p \neq q$. The internal product $*$ on Sym is then defined by requiring that $\alpha$ be an anti-isomorphism, i.e. by

$$
F * G=\alpha^{-1}(\alpha(G) \circ \alpha(F))
$$


for $F, G \in \mathbf{S y m}$. The fundamental property for computing with the internal product is the following formula:

Proposition 2.1. [18] Let $F_{1}, F_{2}, \ldots, F_{r}, G$ be noncommutative symmetric functions. Then,

$$
\left(F_{1} F_{2} \ldots F_{r}\right) * G=\mu_{r}\left[\left(F_{1} \otimes F_{2} \otimes \cdots \otimes F_{r}\right) * \Delta^{r}(G)\right]
$$

where in the right-hand side, $\mu_{r}$ denotes the $r$-fold ordinary multiplication and $*$ stands for the operation induced on $\mathbf{S y m}^{\otimes n}$ by $*$.

\subsection{Quasi-symmetric Functions}

As shown by Malvenuto and Reutenauer [22]. the algebra of noncommutative symmetric functions is in natural duality with the algebra of quasi-symmetric functions, introduced by Gessel [23].

Let $X=\left\{x_{1}, x_{2}, \ldots, x_{n} \ldots\right\}$ be a totally ordered infinite alphabet. An element $f \in \mathrm{C}[X]$ is said to be a quasi-symmetric function iff for any composition $K=\left(k_{1}, \ldots, k_{m}\right)$ and $x_{i}, y_{j} \in X$ such that $y_{1}<y_{2}<\cdots<y_{m}$ and $z_{1}<z_{2}<\cdots<z_{m}$, one has

$$
\left(f \mid y_{1}^{k_{1}} y_{2}^{k_{2}} \ldots y_{m}^{k_{m}}\right)=\left(f \mid z_{1}^{k_{1}} z_{2}^{k_{2}} \ldots z_{m}^{k_{m}}\right)
$$

where $\left(f, \mid x^{K}\right)$ denotes the coefficient of the monomial $x^{K}$ in $f$. The quasi-symmetric functions form a subalgebra of $\mathbf{C}[X]$ denoted by $Q S y m$.

One associates to a composition $I=\left(i_{1}, i_{2}, \ldots, i_{m}\right)$ the quasi-monomial function $M_{I}$ defined by

$$
M_{I}=\sum_{y_{1}<y_{2}<\cdots<y_{m}} y_{1}^{i_{1}} y_{2}^{i_{2}} \ldots y_{m}^{i_{m}}
$$

The family of quasi-monomial functions is clearly a basis of QSym. Another important basis of QSym is formed by the quasi-ribbon functions, defined by

$$
F_{I}=\sum_{J \succeq I} M_{J}
$$

where $\succeq$ is the refinement order (i.e. $J \succeq I$ iff $D(J) \supseteq D(I)$ ). For example, $F_{122}=M_{122}+M_{1112}+$ $M_{1211}+M_{11111}$.

The duality between Sym and QSym is realized by the pairing

$$
\left\langle S^{I}, M_{J}\right\rangle=\delta_{I, J} \quad \text { or } \quad\left\langle R_{I}, F_{J}\right\rangle=\delta_{I, J}
$$

The Hopf algebra QSym can then be identified with the (graded) Hopf algebra dual of Sym.

\subsection{Differences and Products of Alphabets}

We recall here some basic definitions concerning transformations of alphabets. We refer elsewhere [7] for more details. The basic idea is to embed noncommutative symmetric functions in a noncommutative polynomial algebra (for example, by defining $\sigma(A ; t)=\prod_{i \geq 1}\left(1-t a_{i}\right)^{-1}$ for some noncommutative alphabet $A$ ), and then to regard the images of the generators $S_{n}^{-1}(A)$ by an algebra morphism as being the symmetric functions $S_{n}\left(A^{\prime}\right)$ of another alphabet $A^{\prime}$, which can sometimes be explicit, but may also be 
virtual. For example, the formal difference of two genuine alphabets $A$ and $B$ is generally only a virtual alphabet, having nevertheless well-defined symmetric functions, expressible in terms of those of $A$ and $B$.

We first recall the definition of the product of a totally ordered alphabet by a noncommutative alphabet.

Definition 2.2 Let $X$ be a totally ordered commutative alphabet and let $A$ be a noncommutative alphabet. The complete symmetric functions $S_{n}(X A)$ of the alphabet $X A$ are defined by the generating series

$$
\sigma(X A ; t)=\sum_{n \geq 0} S_{n}(X A) t^{n}:=\prod_{x \in X} \sigma(A ; x t)
$$

the product being taken according to the total ordering of $X$.

Example 2.3 Let $X_{q}=1 /(1-q)$ denote the totally ordered alphabet $X_{q}=\left\{\cdots<q^{n}<\cdots<q<1\right\}$. The complete symmetric functions of the alphabet $A /(1-q)$ are

$$
\sigma\left(\frac{A}{1-q} ; t\right)=\sum_{n \geq 0} S_{n}\left(\frac{A}{1-q}\right) t^{n}:=\prod_{n \geq 0}^{\leftarrow} \sigma\left(A ; q^{n} t\right)
$$

We recall the following important property [7]:

Proposition 2.4 Let $X, Y$ be two totally ordered commutative alphabets and let $A$ be a noncommutative alphabet. Then, for any $F_{n}$ of $\mathbf{S y m}_{n}$,

$$
F_{n}((X \times Y) A)=F_{n}(X A) * S_{n}(Y A)
$$

where $X \times Y$ denotes the direct product of the two alphabets $X$ and $Y$ endowed with the lexicographic ordering.

This property suggests the notation $S_{n}(A / X)$ for the $*$-inverse of $S_{n}(X A)$ in $\mathbf{S y m}_{n}$.

Finally, here is the definition of the difference of two noncommutative alphabets.

Definition 2.5 Let $A, B$ be two noncommutative alphabets. The complete symmetric functions $S_{n}(A-B)$ of the alphabet $A-B$ are defined by the generating series

$$
\sigma(A-B ; t)=\sum_{n \geq 0} S_{n}(A-B) t^{n}:=\sigma(B ; t)^{-1} \sigma(A ; t)=\lambda(B ;-t) \sigma(A ; t)
$$

The notation $(1-q) A=A-q A$ therefore denotes the alphabet whose complete symmetric functions are

$$
\sigma((1-q) A ; t)=\sum_{n \geq 0} S_{n}((1-q) A) t^{n}:=\lambda(A ;-q t) \sigma(A ; t)
$$

These notations are coherent since it can be checked that $S_{n}((1-q) A)$ is actually the inverse of $S_{n}(A /(1-$ q)) in $\mathrm{Sym}_{n}$ for the internal product.

\section{Deformations of Cauchy and Convolution Products}

In the sequel, $K$ will denote a field of characteristic 0 , and $A$ will always be an infinite alphabet whose letters are indexed by $\mathbf{N}^{*}$, i.e. $A=\left\{a_{1}, a_{2}, \ldots, a_{n}, \ldots\right\}$. 


\subsection{The General Case}

Consider, for all $n \geq 1$, an invertible element

$$
\beta_{n}=\sum_{\sigma \in \mathfrak{S}_{n}} b_{\sigma}^{(n)} \sigma \in K\left[\mathfrak{S}_{n}\right]
$$

We require that $\beta_{1}=I d_{\mathfrak{S}_{1}}$. This data defines a linear operator $\beta$ on $K\langle A\rangle$ by

$$
\beta\left(a_{i_{1}} \ldots a_{i_{n}}\right)=a_{i_{1}} \ldots a_{i_{n}} \cdot \beta_{n}=\sum_{\sigma \in \mathfrak{S}_{n}} b_{\sigma}^{(n)} a_{i_{\sigma(1)}} \ldots a_{i_{\sigma(n)}}
$$

where $a_{i_{1}}, \ldots, a_{i_{n}} \in A$.

This allows us to equip $K\langle A\rangle$ with a new product $\odot_{\beta}$, defined by

$$
u \odot_{\beta} v=\beta\left(\beta^{-1}(u) \cdot \beta^{-1}(v)\right)
$$

for $u, v$ of $A^{*}$. In other terms, this product is defined in such a way that $\beta$ becomes an isomorphism of algebras between $K\langle A\rangle$ equipped with its usual concatenation (or Cauchy) product and $K\langle A\rangle$ equipped with the new product $\odot_{\beta}$.

Thus, $\left(K\langle A\rangle, \odot_{\beta}\right)$ is a free associative algebra on $\beta(A)=\beta_{1}(A)=A$. It is therefore endowed with a canonical comultiplication $c_{\beta}$, defined by

$$
c_{\beta}(a)=1 \otimes a+a \otimes 1
$$

for $a \in A$, and by the requirement that $c_{\beta}$ is an algebra morphism for $\odot_{\beta}$.

Let $\mathcal{C}_{\beta}(A)$ be the convolution algebra of the Hopf algebra $\left(K\langle A\rangle, \odot_{\beta}, c_{\beta}\right)$, i.e. $\mathcal{C}_{\beta}(A)=$ End $^{\mathrm{gr}} K\langle A\rangle$ endowed with the convolution product

$$
f \otimes \beta g=\mu_{\beta} \circ(f \otimes g) \circ c_{\beta}
$$

where $\mu_{\beta}: u \otimes v \mapsto u \odot_{\beta} v$ is the multiplication. When $\beta=I$, it is well known that the direct sum of the group algebras of all symmetric groups

$$
K[\mathfrak{S}]=\bigoplus_{n \geq 0} K\left[\mathfrak{S}_{n}\right]
$$

is a subalgebra of the convolution algebra (cf. Reutenauer [2]). This is also true for the $\beta$-deformed products.

Proposition 3.1 The $\beta$-convolution algebra $\left(K[\mathfrak{S}], \circledast_{\beta}\right)$ is isomorphic to the usual convolution algebra $(K[\mathfrak{S}], \$)$ (which corresponds to the case where $\beta$ is the identity).

Proof Let $c$ be the comultiplication of $K\langle A\rangle$ (for its usual Cauchy structure) making letters primitive. By definition of $\odot_{\beta}$,

$$
\beta\left(a_{1} \ldots a_{n}\right)=a_{1} \odot_{\beta} \cdots \odot_{\beta} a_{n}
$$

for $a_{i}$ of $A$. Using this property, it is easy to see that the following diagram is commutative: 


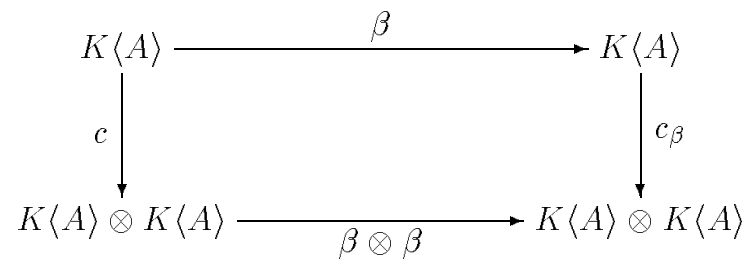

In other words, $c_{\beta}=(\beta \otimes \beta) \circ c \circ \beta^{-1}$, so that

$$
\begin{aligned}
\sigma \otimes \beta \tau & =\odot_{\beta} \circ(\sigma \otimes \tau) \circ c_{\beta} \\
& =\beta \circ \odot \circ\left(\beta^{-1} \otimes \beta^{-1}\right) \circ(\sigma \otimes \tau) \circ(\beta \otimes \beta) \circ c \circ \beta^{-1} \\
& =\beta \circ \odot \circ\left(\left(\beta^{-1} \circ \sigma \circ \beta\right) \otimes\left(\beta^{-1} \circ \tau \circ \beta\right)\right) \circ c \circ \beta^{-1}
\end{aligned}
$$

where we identify an element $x$ of $K[\mathfrak{S}]$ with the endomorphism corresponding to its left action $y \longrightarrow$ $x \circ y$ on $K[\mathfrak{S}](\odot$ denotes here the usual concatenation product of $K\langle A\rangle)$. Consider now the bijection $f_{\beta}$ from $K[\mathfrak{S}]$ into itself defined by

$$
f_{\beta}(\sigma)=\beta_{n} \circ \sigma \circ \beta_{n}^{-1}
$$

for $\sigma \in \mathfrak{S}_{n}$. We have just proved that

$$
f_{\beta}\left(\sigma \circledast_{\beta} \tau\right)=f_{\beta}(\sigma) \circledast f_{\beta}(\tau)
$$

and $f_{\beta}$ is the required isomorphism.

Note 3.2 The definition of $\beta$ shows that

$$
\sigma \circ \beta(\tau)=\beta(\sigma \circ \tau)
$$

for any two permutations $\sigma$ and $\tau$. This just means that the left and right actions of the symmetric group commute. One can then easily check that

$$
\sigma \circledast_{\beta} \tau=\beta(\sigma \circledast \tau)
$$

for permutations $\sigma$ and $\tau$ of arbitrary orders.

Consider now the subalgebra $\Sigma_{\beta}$ of $\left(K[\mathfrak{S}], \otimes_{\beta}\right)$ which is generated by all the elements $I d_{n}=12 \ldots n$ for every $n \geq 0$. When $\beta$ is the identity of $K\langle A\rangle, \Sigma_{\beta}$ is isomorphic to the direct sum $\Sigma$ of all descent algebras equipped with the convolution product (cf. Reutenauer [2]), hence to the algebra of noncommutative symmetric functions (cf. Sect. 2). An explicit isomorphism between these algebras is given by

$$
S_{i_{1}} S_{i_{2}} \ldots S_{i_{n}} \longrightarrow I d_{i_{1}} \circledast I d_{i_{2}} \circledast \cdots \circledast I d_{i_{n}}
$$

One can deform this isomorphism by constructing a new isomorphism denoted $\alpha_{\beta}$ from $\mathbf{S y m}$ into $\Sigma_{\beta}$ which maps the complete function $S^{I}$ (where $I=\left(i_{1}, \ldots, i_{n}\right)$ is a composition) to the convolution product

$$
I d_{i_{1}} \otimes_{\beta} I d_{i_{2}} \otimes_{\beta} \cdots \otimes_{\beta} I d_{i_{n}}
$$


It is interesting to observe that the isomorphism $\alpha_{\beta}$ can be seen as a deformation of the classical interpretation $\alpha$ of noncommutative symmetric functions into Solomon's descent algebra. One can therefore obtain by this method different interpretations of noncommutative symmetric functions. The following result gives an explicit expression for the deformed interpretation map $\alpha_{\beta}$ :

Proposition 3.3 For $F_{n} \in \mathbf{S y m}_{n}$,

$$
\alpha_{\beta}\left(F_{n}\right)=\beta_{n}^{-1} \circ \alpha\left(F_{n}\right) \circ \beta_{n}
$$

Proof With the same notations as in the proof of Proposition 3.1, one has

$$
f_{\beta}\left(\alpha_{\beta}\left(S^{I}\right)\right)=f_{\beta}\left(I d_{i_{1}} \circledast \beta \cdots I_{\beta} \cdots d_{i_{n}}\right)=f_{\beta}\left(I d_{i_{1}}\right) \circledast \cdots f_{\beta}\left(I d_{i_{n}}\right)=\alpha\left(S^{I}\right)
$$

according to (6), and to the fact that $f_{\beta}\left(I d_{k}\right)=I d_{k}$ for every $k \geq 0$. Hence, $f_{\beta}\left(\alpha_{\beta}\left(S^{I}\right)\right)=\alpha\left(S^{I}\right)$. That is, $\alpha_{\beta}\left(S^{I}\right)=\beta_{n}^{-1} \circ \alpha\left(S^{I}\right) \circ \beta_{n}$.

As an immediate consequence, we can state:

Corollary 3.4 The convolution algebra $\Sigma_{\beta}$ is a subalgebra of $K[\mathfrak{S}]$ equiped with the usual composition product.

Proof Let $x$ and $y$ be two homogenous elements of the same order $n$ of $\Sigma_{\beta}$. By construction, there exists two elements $f$ and $g$ of $\mathbf{S y m}_{n}$ such that $x=\alpha_{\beta}(f)$ and $y=\alpha_{\beta}(g)$. It follows then from Proposition 3.3 that

$$
\begin{aligned}
x \circ y & =\left(\beta_{n}^{-1} \circ \alpha(f) \circ \beta_{n}\right) \circ\left(\beta_{n}^{-1} \circ \alpha(g) \circ \beta_{n}\right) \\
& =\beta_{n}^{-1} \circ \alpha(f) \circ \alpha(g) \circ \beta_{n} \\
& =\beta_{n}^{-1} \circ \alpha(g * f) \circ \beta_{n} \\
& =\alpha_{\beta}(g * f) \in \Sigma_{\beta}
\end{aligned}
$$

Note 3.5 The proof of the corollary shows that

$$
\alpha_{\beta}(F) \circ \alpha_{\beta}(G)=\alpha_{\beta}(G * F)
$$

for homogenous elements $F, G$ of the same weight of Sym. It follows in particular that the image by $\alpha_{\beta}$ of a homogenous idempotent of Sym (for the internal product) is still an idempotent in $\Sigma_{\beta}$.

Example 3.6 Let us explicit the interpretation of the image by $\alpha_{\beta}$ of the Eulerian idempotent $\phi_{n}$ (which is the image by $\alpha$ of the element $\Phi_{n} / n$ of $\mathbf{S y m}_{n}$ ). Let $\mathcal{L}$ denote the image of the free Lie algebra $L(A)$ by $\beta$. Transporting by $\beta$ the Poincaré-Birkhoff-Witt decomposition of $K\langle A\rangle$, we obtain

$$
K\langle A\rangle=K \oplus \mathcal{L} \oplus(\mathcal{L}, \mathcal{L})_{\beta} \oplus \ldots \oplus(\underbrace{\mathcal{L}, \ldots, \mathcal{L}}_{n \text { terms }})_{\beta} \oplus \ldots
$$

where

$$
\left(x_{1}, \ldots, x_{n}\right)_{\beta}=\frac{1}{n !}\left(\sum_{\sigma \in \mathcal{\Theta}_{n}} x_{\sigma(1)} \odot_{\beta} \cdots \odot_{\beta} x_{\sigma(n)}\right)
$$

for $x_{1}, \ldots, x_{n} \in K\langle A\rangle$. Then, $\alpha_{\beta}\left(\Phi_{n} / n\right)=\beta_{n} \circ \phi_{n} \circ \beta_{n}$ is the idempotent corresponding to the projection of the homogenous component of degree $n$ of $\mathcal{L}$ with respect to the above direct sum decomposition of $K\langle A\rangle$. 
Note 3.7 In many interesting cases, the elements $\beta_{n}=\beta_{n}(q)$ depend on some parameter $q$ and are invertible for generic values of $q$. In such situations, the convolution algebra $\Sigma_{\beta}(q)$ degenerates when $q$ takes a value $q_{0}$ for which $\beta=\beta(q)$ is not an isomorphism. We will, however, still use the notation $\Sigma_{\beta}\left(q_{0}\right)$ to denote the limit of $\Sigma_{\beta}(q)$ for $q \rightarrow q_{0}$ whenever it exists. Several interesting problems arise in the investigation of these degenerate convolution algebras.

Note 3.8 The framework presented here can be easily generalized to some other situations. Among them is the case of the so-called orthogonal Lie idempotent $[9,2]$. The orthogonal Lie idempotent $\pi_{n}$ is the idempotent of $\mathrm{Q}\left[\mathfrak{S}_{n}\right]$ which corresponds to the orthogonal projection from $K\langle A\rangle$ (endowed with its standard scalar product for which words form an orthonormal basis) onto the homogenous component $L(A)_{n}$ of order $n$ of the free Lie algebra $L(A) . \pi_{n}$ is also the projection onto $L(A)_{n}$ with respect to the decomposition of $K\langle A\rangle$ given by Ree's theorem, i.e.

$$
K\langle A\rangle=K \oplus L(A) \oplus L(A) \amalg L(A) \oplus \ldots \oplus \underbrace{L(A) \amalg \ldots \amalg L(A)}_{n \text { terms }} \oplus \ldots
$$

where $\amalg$ denotes the usual shuffle product on $\mathbf{Q}\langle A\rangle$.

Let $\mathcal{B}$ be any linear basis of $L(A)$. The shuffle algebra $(\mathrm{Q}\langle A\rangle, \amalg)$ is a free commutative algebra with $\mathcal{B}$ as generating family (cf. Reutenauer [2]). This property allows us to define a comultiplication $c_{\uplus}$ on $\mathrm{Q}\langle A\rangle$ by

1. $c_{\uplus}(L)=1 \otimes L+L \otimes 1$ for every Lie element $L \in L(A)$;

2. $c_{\uplus}(P \amalg Q)=c_{\uplus}(P)(\amalg \otimes Ш) c_{\Psi}(Q)$ for every polynomials $P, Q \in \mathbf{Q}\langle A\rangle$.

One can then consider the associated convolution product $\otimes_{\Perp}$ on Q[S], defined by

$$
\sigma \otimes_{ш} \tau=Ш \circ(\sigma \otimes \tau) \circ c_{\uplus}(12 \ldots n+m)
$$

for $\sigma \in \mathfrak{S}_{n}$ and $\tau \in \mathfrak{S}_{m}$. The commutativity of the shuffle product implies the cocommutativity of $c_{\uplus}$. Hence the convolution algebra $\left(\mathbf{Q}[\mathfrak{S}], \circledast_{\uplus}\right)$ is here commutative. It follows that its subalgebra $\Sigma_{\Psi}$ generated by the identity elements of all symmetric groups is also commutative. Consider now the morphism from Sym into $\Sigma_{\uplus}$ defined as in the general case by

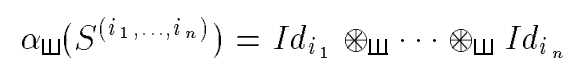

This is a degenerate situation in which the image by $\alpha_{\uplus}$ of the algebra of noncommutative symmetric functions is not isomorphic to Solomon's descent algebra. The generic interpretation of the image by $\alpha_{\amalg}$ of the Eulerian idempotent $\Phi_{n}$ given in Example 3.6 is, however, still valid here. It follows from this interpretation that Ree's decomposition is equivalent to

$$
\alpha_{\uplus}\left(\Phi_{n}\right)=n \cdot \pi_{n}
$$

It follows that $\pi_{n}$ belongs to the homogenous component $\Sigma_{\Psi}^{(n)}$ of order $n$ of $\Sigma$. It is easy to see that this set is a subalgebra of $K\left[\mathfrak{S}_{n}\right]$ of dimension $p(n)$ (the number of partitions of $n$ ). An interesting question would be to characterize this subalgebra and to give explicit formulas for the images of the standard bases of Sym by $\alpha_{\amalg}$. The decomposition of $\pi_{n}$ on such bases would then be immediately given by decomposition relations of $\Phi_{n}$ in Sym. 
Example 3.9 Let us describe $\Sigma_{\Psi}^{(n)}$ for $n=2$ and $n=3$. In the first case, $\Sigma_{\Psi}^{(2)}$ is just the descent algebra $\Sigma_{2}=\mathrm{Q}\left[\mathfrak{S}_{2}\right]$. In the second case, $\Sigma_{\uplus}^{(3)}$ is the commutative algebra spanned by

$$
\alpha_{\uplus}\left(R_{3}\right)=123, \alpha_{\uplus}\left(R_{12}\right)=\alpha_{\uplus}\left(R_{21}\right)=\frac{1}{2}(132+213+231+312), \alpha_{\uplus}\left(R_{111}\right)=321
$$

and the orthogonal projector is

$$
\pi_{3}=\alpha_{\uplus}\left(\Phi_{3}\right)=\alpha_{\uplus}\left(R_{3}-\frac{1}{2} R_{21}-\frac{1}{2} R_{21}+R_{111}\right)=123-\frac{1}{2}(132+213+231+312)+321
$$

It is also interesting to see that the image by $\alpha_{\varpi}$ of the homogenous component $L_{n}(\Psi)$ of order $n$ of the free Lie algebra $L(\Psi) \subset$ Sym generated by the family $\left(\Psi_{n}\right)_{n \geq 1}$ (or equivalently by the family $\left(\Phi_{n}\right)_{n \geq 1}$ ) collapses here onto a line, which is necessarily equal to $\mathbf{Q} \pi_{n}$.

\subsection{Deformations Using Noncommutative Symmetric Functions}

Here is an interesting special case of the previous constructions. Let $F=\left(F_{n}\right)_{n \geq 1}$ be a family of elements of $\mathbf{S y m}_{n}$, with $F_{1}=S_{1}$. We assume that every $F_{n}$ is invertible for the internal product of $\mathbf{S y m}_{n}$. We can then consider the bijection $\beta_{n}$ defined by

$$
\beta_{n}=\alpha\left(F_{n}\right)
$$

where we identify an element of $K\left[\mathfrak{S}_{n}\right]$ with the linear morphism defined by its right action. In other words, $\beta_{n}$ is given by

$$
\beta_{n}: x \in K\left[\mathfrak{S}_{n}\right] \longrightarrow x \circ \alpha\left(F_{n}\right)
$$

Denote by $\odot_{F}$ the product of $K\langle A\rangle$ associated with the family $\left(\beta_{n}\right)_{n \geq 1}$ by the above construction. We also denote by $\oiint_{F}$ and $\alpha_{F}$ the corresponding convolution product and interpretation morphism (of Sym into the convolution algebra $\Sigma_{F}=\Sigma_{\beta}$ ).

Note first that Proposition 3.3 shows that the image of $\alpha_{F}$ is here exactly Solomon's descent algebra. Using $\alpha^{-1}$, we can therefore reinterpret the convolution product $\otimes_{F}$. Formula (7) shows in particular that the algebra $\left(\Sigma, \circledast_{F}\right)$ is isomorphic to the algebra of noncommutative symmetric functions endowed with the $F$-product defined by

$$
U *_{F} V=F_{n+m} *(U V)
$$

for homogenous elements $U$ and $V$ of weight $n$ and $m$, respectively. Identifying again $\alpha(U)$ with $U$ and applying Proposition 3.3, one has has

$$
\alpha_{F}(U)=F_{n} * U * F_{n}^{*(-1)}
$$

for $U \in \mathbf{S y m}_{n}$, where $F_{n}^{*(-1)}$ denotes the inverse of $F_{n}$ for the internal product of $\mathbf{S y m}_{n}$.

We will study in the final section of this paper the situations corresponding to the families given by the $q$-bracketing and its inverse, i.e. the cases $F_{n}=S_{n}((1-q) A)$ and $F_{n}=S_{n}(A /(1-q))$.

\section{The $q$-shuffle Product}

We present in this section the $q$-shuffle product which is an interesting deformation of the usual Cauchy product in $K\langle A\rangle$. We first give the formal definition of this product and then show that this deformation is a special case of the general framework introduced in Sect. 3. 


\subsection{Definition}

The shuffle product can be recursively defined by the formula

$$
a u \amalg b v=a(u \amalg b v)+b(a u \amalg v)
$$

where $a, b \in A$ and $u, v \in A^{*}$. Inserting a power of an indeterminate $q$ in this definition, one obtains an interesting deformation, which turns out to be a particular case of a construction of Rosso [24].

Definition 4.1 The q-shuffle product is the bilinear operation $\odot_{q}$ on $\mathbf{N}[q]\langle A\rangle$ recursively defined by

$$
\begin{gathered}
1 \odot_{q} u=u \odot_{q} 1=u \\
(a u) \odot_{q}(b v)=a\left(u \odot_{q} b v\right)+q^{|a u|} b\left(a u \odot_{q} v\right)
\end{gathered}
$$

where $u, v\left(\right.$ resp. a, b) are words (resp. letters) of $A^{*}$ (resp. A)

This operation interpolates between the concatenation product (for $q=0$ ) and the usual shuffle product (for $q=1$ ) on $\mathbf{N}[q]\langle A\rangle$. The following property is a particular case of a result proved in Sect. 6 .

Proposition 4.2 The q-shuffle product is associative.

As an exercise, let us check it directly. It is clearly sufficient to prove that

$$
\left(a u \odot_{q} b v\right) \odot_{q} c w=a u \odot_{q}\left(b v \odot_{q} c w\right)
$$

for $u, v, w \in A^{*}$ and $a, b, c \in A$. Applying (11), one finds

$$
\begin{aligned}
\left(a u \odot_{q} b v\right) \odot_{q} c w= & a\left(u \odot_{q} b v\right) \odot_{q} c w+q^{|a u|} b\left(a u \odot_{q} v\right) \odot_{q} c w \\
= & a\left(\left(u \odot_{q} b v\right) \odot_{q} c w\right)+q^{|a u|+|b v|} c\left(a\left(u \odot_{q} b v\right) \odot_{q} w\right) \\
& +q^{|a u|} b\left(\left(a u \odot_{q} v\right) \odot_{q} c w\right)+q^{2|a u|+|b v|} c\left(b\left(a u \odot_{q} v\right) \odot_{q} w\right) \\
= & a\left(\left(u \odot_{q} b v\right) \odot_{q} c w\right)+q^{|a u|} b\left(\left(a u \odot_{q} v\right) \odot_{q} c w\right) \\
& +q^{|a u|+|b v|} c\left(\left(a\left(u \odot_{q} b v\right)+q^{|a u|} b\left(a u \odot_{q} v\right)\right) \odot_{q} w\right)
\end{aligned}
$$

so that

$$
\begin{aligned}
\left(a u \odot_{q} b v\right) \odot_{q} c w= & a\left(\left(u \odot_{q} b v\right) \odot_{q} c w\right) \\
& +q^{|a u|} b\left(\left(a u \odot_{q} v\right) \odot_{q} c w\right)+q^{|a u|+|b v|} c\left(\left(a u \odot_{q} b v\right) \odot_{q} w\right)
\end{aligned}
$$

On the other hand,

$$
\begin{aligned}
a u \odot_{q}\left(b v \odot_{q} c w\right)= & a u \odot_{q}\left(b\left(v \odot_{q} c w\right)+q^{|b v|} c\left(b v \odot_{q} w\right)\right) \\
= & a\left(u \odot_{q} b\left(v \odot_{q} c w\right)\right)+q^{|a u|} b\left(a u \odot_{q}\left(v \odot_{q} c w\right)\right) \\
& +q^{|b v|} a\left(u \odot_{q} c\left(b v \odot_{q} w\right)\right)+q^{|a u|+|b v|} c\left(a u \odot_{q}\left(b v \odot_{q} w\right)\right) \\
= & a\left(u \odot_{q}\left(b\left(v \odot_{q} c w\right)+q^{|b v|} c\left(b v \odot_{q} w\right)\right)\right) \\
& +q^{|a u|} b\left(a u \odot_{q}\left(v \odot_{q} c w\right)\right)+q^{|a u|+|b v|} c\left(a u \odot_{q}\left(b v \odot_{q} w\right)\right)
\end{aligned}
$$


It follows that

$$
\begin{aligned}
a u \odot_{q}\left(b v \odot_{q} c w\right)= & a\left(u \odot_{q}\left(b v \odot_{q} c w\right)\right) \\
& +q^{|a u|} b\left(a u \odot_{q}\left(v \odot_{q} c w\right)\right)+q^{|a u|+|b v|} c\left(a u \odot_{q}\left(b v \odot_{q} w\right)\right)
\end{aligned}
$$

which implies the result by induction.

\subsection{The Operator $U(q)$}

As already observed, the $q$-shuffle can also be interpreted as a deformation (in the sense of the deformation theory of algebras) of the concatenation product of a free associative algebra. It is known that these algebras are rigid, which implies that for generic $q$ the $q$-shuffle product is necessarily a deformation of the concatenation product in the sense of Sect. 3. It is easy to exhibit the conjugation isomorphism. Let $U(q)$ be the endomorphism of $\mathbf{Z}(q)\langle A\rangle$ defined by

$$
U(q)\left(a_{1} a_{2} \ldots a_{n}\right)=a_{1} \odot_{q} a_{2} \odot_{q} \ldots \odot_{q} a_{n}
$$

for $w=a_{1} a_{2} \ldots a_{n}$ of $A^{*}$. The product $\odot_{q}$ being multihomogeneous, the restriction of $U(q)$ to $\mathbf{Z}(q)\langle A\rangle_{\lambda}$ defines an endomorphism $U(q)_{\lambda}$ of $\mathbf{Z}(q)\langle A\rangle_{\lambda}$ for each multi-degree $\lambda$, and one can write

$$
U(q)=\bigoplus_{\lambda} U(q)_{\lambda}
$$

Moreover, $U(q)$ clearly commutes with letter to letter substitutions. This shows that one can recover $U(q)$ from its restriction to standard words $U(q)_{1^{n}}$. This endomorphism corresponds to the right action of an element $U_{n}(q)$ of $\mathbf{Z}[q]\left[\mathfrak{S}_{n}\right]$, which is given by the following formula:

Proposition 4.3

$$
U_{n}(q)(12 \ldots n)=1 \odot_{q} 2 \odot_{q} \cdots \odot_{q} n=\sum_{\sigma \in \mathfrak{S}_{n}} q^{\ell(\sigma)} \sigma
$$

This formula follows by induction from the following one, itself established by induction:

\section{Lemma 4.4}

$$
12 \ldots n-1 \odot_{q} n=\sum_{i=0}^{n-1} q^{n-1-i}(1 \ldots i n i+1 \ldots n-1)
$$

It follows that for a word of length $n$,

$$
U(q)\left(a_{1} a_{2} \ldots a_{n}\right)=a_{1} a_{2} \ldots a_{n} \cdot U_{n}(q)
$$

and one sees that the $q$-shuffle is a deformation of the Cauchy product in the sense of Sect. 3 whenever $U_{n}(q)$ is a bijection.

Indeed, $\operatorname{det} U_{n}(q)$ is an analytic function of $q$, and $U_{n}(0)$ being the identity of $\mathbf{Z}\left[\mathfrak{S}_{n}\right], U_{n}(q)$ is invertible for small complex values of $q$, and thus also for generic $q$ (that is, for $q$ an indeterminate, or for $q$ a complex number avoiding a discrete set of values). It follows that $U(q)$ itself is also generically invertible, and hence that the $q$-shuffle is generically a deformation of the Cauchy product in the sense of Sect. 3 . One can restate this result as follows: 
Proposition 4.5 For generic $q, U(q)$ is an isomorphism between the concatenation algebra $(\mathbf{Z}(q)\langle A\rangle, \cdot)$ and the $q$-shuffle algebra $\left(\mathbf{Z}(q)\langle A\rangle, \odot_{q}\right)$.

Equivalently, when $U(q)$ is invertible,

$$
x \odot_{q} y=U(q)\left(U(q)^{-1}(x) \cdot U(q)^{-1}(y)\right)
$$

\subsection{U(q) in Physics: The Quon Algebra}

The problem arises now of finding the values of $q$ for which $U_{n}(q)$ is actually invertible. It turns out that this problem has already been solved by Zagier [25] and by Bożeijko and Speicher [12] in a totally different contexts. The starting point of: Zagier [25] was a problem in physics, related to a model of quantum field thenry allowing the existence of particles ('quons') displaying small violations of Bose or Fermi statistics [11]. Classically, bosons and fermions are described by creation and annihilation operators $a_{i}, a_{j}^{*}$ satisfying canonical commutation or anticommutation relations. Here, the problem was to determine whether it was possible to realize the $q$-commutator (' $q$-mutator') relations

$$
a_{i} a_{j}^{*}-q a_{j}^{*} a_{i}=\delta_{i, j} \quad(i, j \geq 1)
$$

by operators $a_{i}, a_{j}^{*}$ of a Hilbert space $H$, such that $a_{i}^{*}$ be the adjoint of $a_{i}$, and such that there exists a distinguished vector $|0\rangle \in H$ (the vacuum state) annihilated by all the $a_{i}$

$$
a_{i}|0\rangle=0 \quad \text { for all } i
$$

Zagier proved the realizability of this model for $-1<q<1$, and the same result was obtained independently by Bożejko and Speicher, who encoutered the same algebra in their analysis of a generalization of Brownian motion. Another proof (with a gap) appears in Fivel [26] - see also the erratum [27].

It is easy to see that the realizability problem can be reduced to the case where $H$ is equal to the vector space $H(q)$ generated by the images of $|0\rangle$ under all products of $a_{k}$ and $a_{k}^{*}$. This space has a basis consisting of all states

$$
|K\rangle=a_{k_{1}}^{*} \ldots a_{k_{n}}^{*}|0\rangle
$$

for $K=\left(k_{1}, \ldots, k_{n}\right) \in\left(\mathbf{N}^{*}\right)^{n}$. Consider now the infinite matrix $A(q)$ defined by

$$
A(q)=(\langle K \mid L\rangle)_{K, L \in\left(\mathbf{N}^{*}\right)^{n}, n \geq 0}
$$

where the scalar product is defined by the condition $\langle 0 \mid 0\rangle=1$. The Hilbert space realizability of relations (18) is equivalent to the positive definiteness of the matrix $A(q)$. Moreover, one can prove that this condition is equivalent to the positive definiteness of all submatrices of $A(q)$ indexed by permutations of $\mathfrak{S}_{n}$. An easy computation gives

$$
\langle\sigma \mid \tau\rangle=q^{\ell\left(\tau^{-1} \sigma\right)}
$$

which is the matrix of $U_{n}(q)$ in the regular representation.

Hence, one has to prove that all operators $U_{n}(q)$ are positive definite for $-1<q<1$. By continuity, since $U_{n}(0)$ is the identity, it is sufficient to show that $U_{n}(q)$ is non singular in this range. This reduces the realizability problem to the invertibility of the $q$-shuffle operator for $-1<q<1$. This will follow from the computations of the forthcoming section, as well as the complete determination of the values for which $U_{n}(q)$ is invertible. 


\subsection{Zagier's Inversion of $U(q)$}

Define

$$
T_{n}(q)=12 \ldots n-1 \odot n=\sum_{i=0}^{n-1} q^{n-1-i}(1 \ldots \text { in } i+1 \ldots n-1)
$$

(by Lemma 4.4). Embedding $\mathfrak{S}_{n-1}$ in $\mathfrak{S}_{n}$, one can write

$$
U_{n}(q)=\left(1 \odot_{q} \cdots \odot_{q} n-1\right) \odot_{q} n=U_{n-1}(q) T_{n}(q)
$$

so that $U_{n}(q)=T_{2}(q) T_{3}(q) \cdots T_{n}(q)$. Taking reduced decompositions of the factors, we obtain:

Proposition 4.6 For $n \geq 2$, one has the factorization

$$
\begin{aligned}
U_{n}(q)= & \left(1 \odot_{q} 2\right)\left(12 \odot_{q} 3\right) \ldots\left(12 \ldots n-1 \odot_{q} n\right) \\
= & \left(1+q \sigma_{1}\right)\left(1+q \sigma_{2}+q^{2} \sigma_{2} \sigma_{1}\right) \ldots \\
& \left(1+q \sigma_{n-1}+q^{2} \sigma_{n-1} \sigma_{n-2}+\cdots+q^{n-1} \sigma_{n-1} \ldots \sigma_{1}\right)
\end{aligned}
$$

Thus, $U_{n}(q)$ will be invertible if and only if all the $T_{i}(q)$ are invertible for $i \leq n$. These elements can themselves be factorized. To this purpose, we need to introduce two elements $G_{n}(q)$ and $D_{n}(q)$ of $\mathbf{Z}(q)\left[\mathfrak{S}_{n}\right]$, which are defined by induction. One first sets

$$
G_{1}(q)=D_{1}(q)=1
$$

Let now $n \geq 2$, and suppose that $G_{n-1}(q)$ and $D_{n-1}(q)$ are defined. Denote by $g_{n-1}(q)$ and $d_{n-1}(q)$ the images of these elements in $\mathbf{Z}(q)\left[\mathfrak{S}_{n}\right]$ where we identify $\mathfrak{S}_{n-1}$ with the stabilizer of 1 in $\mathfrak{S}_{n}$. That is,

$$
g_{n-1}(q)=\eta_{n-1}^{n}\left(G_{n-1}(q)\right) \quad \text { and } \quad d_{n-1}(q)=\eta_{n-1}^{n}\left(D_{n-1}(q)\right)
$$

where $\eta_{n-1}^{n}$ is the group morphism of $\mathfrak{S}_{n-1}$ into $\mathfrak{S}_{n}$ defined by

$$
\eta_{n-1}^{n}(\sigma)=(1 \sigma(1)+1 \ldots \sigma(n-1)+1)
$$

for $\sigma \in \mathfrak{S}_{n-1}$. Then one defines

$$
\left\{\begin{array}{l}
G_{n}(q)=\left(1-q^{n} \gamma_{n}\right) g_{n-1}(q) \\
D_{n}(q)=d_{n-1}(q)\left(1-q^{n-1} \delta_{n}\right)^{-1}
\end{array}\right.
$$

where

$$
\gamma_{n}=(n-112 \ldots n-2 n) \quad \text { and } \quad \delta_{n}=\left(\begin{array}{lll}
n & 1 & 2
\end{array} \ldots n-1\right)
$$

The complete factorization of $T_{n}(q)$ is then given by

Proposition 4.7 [25] for all $n \geq 1$,

$$
T_{n}(q)=G_{n}(q) D_{n}(q)
$$


Proof Let $n \geq 2$ and suppose by induction and that the formula is valid for $T_{n-1}(q)$. Then,

$$
\begin{aligned}
G_{n}(q) D_{n}(q) & =\left(1-q^{n} \gamma_{n}\right) g_{n-1}(q) d_{n-1}(q)\left(1-q^{n-1} \delta_{n}\right)^{-1} \\
& =\left(1-q^{n} \gamma_{n}\right) t_{n-1}(q)\left(1-q^{n-1} \delta_{n}\right)^{-1}
\end{aligned}
$$

where $t_{n-1}(q)=\eta_{n-1}^{n}\left(T_{n-1}(q)\right)$. It suffices therefore to check that

$$
T_{n}(q)\left(1-q^{n-1} \delta_{n}\right)=\left(1-q^{n} \gamma_{n}\right) t_{n-1}(q)
$$

which follows from a straightforward computation.

Example 4.8 For $n \leq 4$, this gives

$$
\begin{aligned}
T_{2}(q)= & \left(1-q^{2}\right)(1-q(21))^{-1} \\
T_{3}(q)= & \left(1-q^{3}(213)\right)\left(1-q^{2}\right)(1-q(132))^{-1}\left(1-q^{2}(312)\right)^{-1} \\
T_{4}(q)= & \left(1-q^{4}(3124)\right)\left(1-q^{3}(1324)\right)\left(1-q^{2}\right) \\
& (1-q(1243))^{-1}\left(1-q^{2}(1423)\right)^{-1}\left(1-q^{3}(4123)\right)^{-1}
\end{aligned}
$$

It is now easy to obtain an explicit formula for $T_{n}(q)^{-1}$ (which is a factorization of this element into $2 n-3$ terms). To state it, we need to introduce the elements $G_{n}^{\prime}(q)$ and $D_{n}^{\prime}(q)$ defined by $G_{1}^{\prime}(q)=$ $D_{1}^{\prime}(q)=1$ and by the recursive formulas

$$
\left\{\begin{array}{c}
G_{n}^{\prime}(q)=\left(1-q^{n-1} \gamma_{n}\right) g_{n-1}^{\prime}(q) \\
D_{n}^{\prime}(q)=d_{n-1}^{\prime}(q)\left(1+q^{n} \delta_{n}+q^{2 n}\left(\delta_{n}\right)^{2}+\cdots+q^{(n-2) n}\left(\delta_{n}\right)^{n-2}\right)
\end{array}\right.
$$

where $g_{n-1}^{\prime}(q)=\eta_{n-1}^{n}\left(G_{n-1}^{\prime}\right)$ and $d_{n-1}^{\prime}(q)=\eta_{n-1}^{n}\left(D_{n-1}^{\prime}\right)$.

Proposition 4.9 [25] If $q$ is an indeterminate, $T_{n}(q)$ is invertible, and its inverse is

$$
T_{n}(q)^{-1}=\frac{1}{\left(1-q^{2}\right)\left(1-q^{6}\right) \ldots\left(1-q^{n(n-1)}\right)} D_{n}^{\prime}(q) G_{n}^{\prime}(q)
$$

Proof It suffices to find the inverses of the linear factors, which are given by

$$
\left(1-q^{n} \delta_{n}\right)^{-1}=\frac{1}{1-q^{n(n-1)}}\left(1+q^{n} \delta_{n}+q^{2 n}\left(\delta_{n}\right)^{2}+\cdots+q^{(n-2) n}\left(\delta_{n}\right)^{n-2}\right)
$$


Example 4.10 For $n=2,3,4$, one has

$$
\begin{aligned}
T_{2}(q)^{-1}= & \frac{1}{1-q^{2}}(1-q(21)) \\
T_{3}(q)^{-1}= & \frac{1}{\left(1-q^{2}\right)\left(1-q^{6}\right)}\left(1-q^{2}(312)\right)(1-q(132))\left(1+q^{3}(213)\right) \\
T_{4}(q)^{-1}= & \frac{1}{\left(1-q^{2}\right)\left(1-q^{6}\right)\left(1-q^{12}\right)}\left(1-q^{3}(4123)\right)\left(1-q^{2}(1423)\right) \\
& (1-q(1243))\left(1+q^{3}(1324)\right)\left(1+q^{4}(3124)+q^{8}(2314)\right)
\end{aligned}
$$

We can now describe the exact values of $q$ for which the operator $U_{n}(q)$ is not invertible.

Corollary 4.11 [25] For every $n \geq 1$, the operators $U_{n}(q)$ and $T_{n}(q)$ are invertible iff $q$ is not a $k(k-1)$ root of unity for some $k \in[1, n]$.

Corollary 4.12 [25] The operator $U(q)$ is invertible when q is not a root of unity.

We have also the following result of Zagier, which is also a special case of a general formula due to Varchenko (cf. [13], Theorem 1.1 or Sect. 6.4).

Corollary $4.13[25,13]$ The determinant of $U_{n}(q)$ considered as an operator for the regular representation of $\mathfrak{S}_{n}$ is

$$
\operatorname{det} U_{n}(q)=\prod_{k=2}^{n}\left(1-q^{k(k-1)}\right)^{(n-k+1) n ! /(k(k-1))}
$$

Proof Using the factorization of $T_{n}(q)$ and the the fact that $\operatorname{det}(1-q \gamma)=\left(1-q^{l}\right)^{n ! / l}$ if $\gamma$ is a cycle of order $l$ in $\mathfrak{S}_{n}$, we obtain

$$
\operatorname{det} T_{i}(q)=\prod_{k=2}^{i}\left(1-q^{k(k-1)}\right)^{n ! /(k(k-1))}
$$

\subsection{Representation Theoretical Interpretation of the $q$-shuffle}

The 0-Hecke algebra $H_{n}(0)$ is the C-algebra obtained by specialization of the generic Hecke algebra $H_{n}(q)$ at $q=0$. It is generated by elements $T_{1}, T_{2}, \ldots, T_{n-1}$ and has the following presentation:

$$
\begin{array}{cl}
T_{i}^{2}=-T_{i} & \text { for } i \in[1, n-1] \\
T_{i} T_{j}=T_{j} T_{i} & \text { for }|i-j|>1 \\
T_{i} T_{i+1} T_{i}=T_{i+1} T_{i} T_{i+1} & \text { for } i \in[1, n-2]
\end{array}
$$

For generic values of $q$, the Hecke algebra is semi-simple, and isomorphic to $\mathbf{C} \mathfrak{S}_{n}$. This is not the case when $q=0$. In particular, the families of irreducible and indecomposable $H_{n}(0)$-modules are not equal.

The irreducible $H_{n}(0)$-modules are 1-dimensional, and parametrized by subsets of $1, \ldots, n-1$ [14]. To see this, it is sufficient to observe that $\left(T_{i} T_{i+1}-T_{i+1} T_{i}\right)^{2}=0$. Thus, all the commutators $\left[T_{i}, T_{j}\right]$ are in 
the radical. But the quotient of $H_{n}(0)$ by the ideal generated by these elements is the commutative algebra generated by $n-1$ elements $t_{1}, \ldots, t_{n-1}$ subject to $t_{i}^{2}=-t_{i}$. It is easy to check that this algebra has no nilpotent elements, so that it is $H_{n}(0) /\left(\operatorname{rad} H_{n}(0)\right)$. The irreducible representations are thus obtained by sending a set of generators to $(-1)$ and its complement to 0 . For reasons that will become transparent later, it is better to label these representations by compositions rather than by subsets. Let $I=\left(i_{1}, \ldots, i_{r}\right)$ be a composition of $n$ and let $D(I)$ the associated subset of [1,n-1]. The irreducible (1-dimensional) representation $\varphi_{I}$ of $H_{n}(0)$ is defined by

$$
\varphi_{I}\left(T_{i}\right)=\left\{\begin{array}{cc}
-1 & \text { if } i \in D(I) \\
0 & \text { if } i \notin D(I)
\end{array}\right.
$$

and the associated $H_{n}(0)$-module will be denoted by $\mathbf{C}_{I}$.

Now let $M$ be an arbitrary finite dimensional $H_{n}(0)$-module and consider a composition series of $M$, i.e. a decreasing sequence

$$
M_{1}=M \supset M_{2} \supset \cdots \supset M_{k} \supset M_{k+1}=\{0\}
$$

of $H_{n}(0)$-modules where each $M_{i} / M_{i+1}$ is irreducible. There exists therefore for each $i \in[1, k]$ a composition $I_{i}$ of $n$ such that $M_{i} / M_{i+1} \simeq \mathrm{C}_{I_{i}}$. The Jordan-Hölder theorem ensures that the quasisymmetric function

$$
\mathcal{F}(M)=\sum_{i=1}^{k} F_{I_{i}}
$$

is independent of the choice of the composition series. The quasi-symmetric function associated with $M$ is called the characteristic of $M$. One can show that it has several properties in common with the usual Frobenius characteristic of a $\mathfrak{S}_{n}$-module [15]. In particular, the characteristic of an induced module

$$
M=\mathbf{C}_{I_{1}} \otimes \mathbf{C}_{I_{2}} \otimes \cdots \otimes \mathbf{C}_{I_{r}} \uparrow_{H_{n_{1}} \otimes H_{n_{2}} \otimes \cdots \otimes H_{n_{r}}}^{H_{n_{1}}+\cdots+n_{r}}
$$

is the product of the characteristics of the factors [15]

$$
\mathcal{F}(M)=F_{I_{1}} F_{I_{2}} \cdots F_{I_{r}}
$$

These induced modules are cyclic, with generator the basis vector $e=1 \otimes 1 \otimes \cdots \otimes 1$ of the onedimensional space $\mathbf{C}_{I_{1}} \otimes \mathbf{C}_{I_{2}} \otimes \cdots \otimes \mathbf{C}_{I_{r}}$. The length filtration

$$
H_{n}(0)^{(k)}=\bigoplus_{\ell(w) \geq k} \mathbf{C} T_{w}
$$

of the 0-Hecke algebra induces a filtration of $M$

$$
M^{(k)}=H_{n}(0)^{(k)} e
$$

and this suggest the definition of a graded characteristic (for these particular modules) by

$$
\mathcal{F}_{q}(M)=\sum_{k \geq 0} q^{k} \mathcal{F}\left(M^{(k)} / M^{(k+1)}\right)
$$




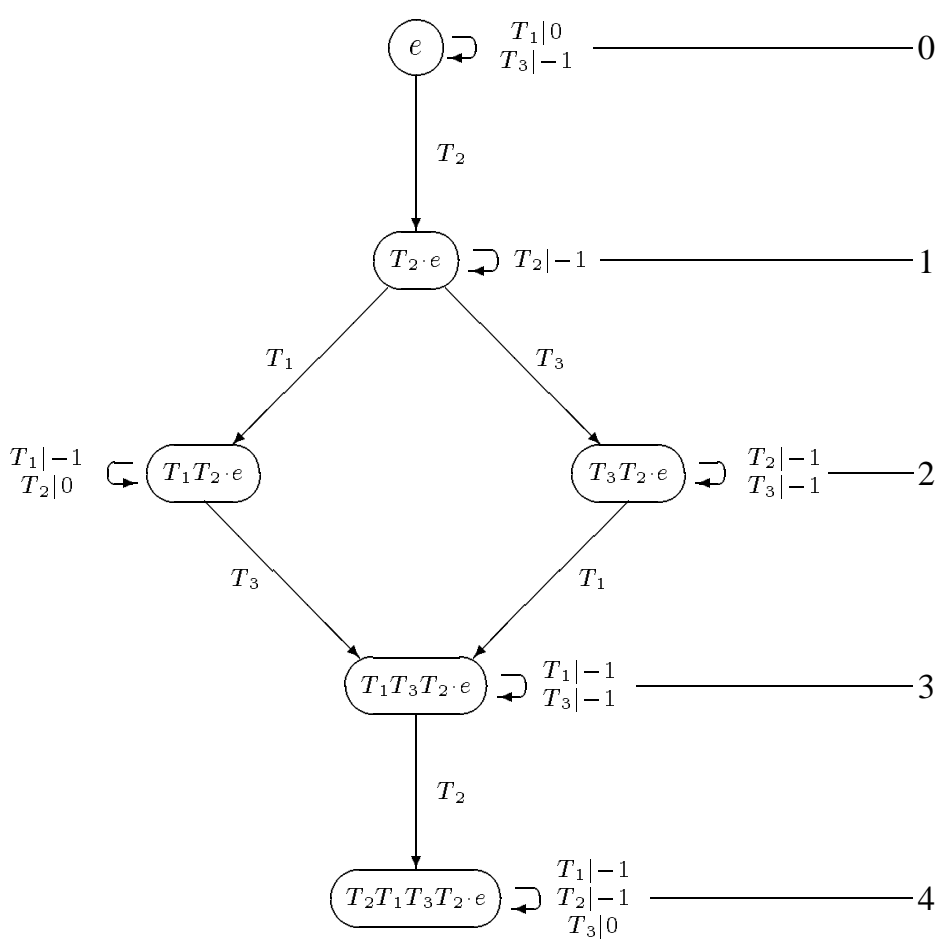

Fig. 1: The induced $H_{4}(0)$-module $M_{((2),(1,1))}$.

Example 4.14 Take $I=(2), J=(1,1)$ and let $M_{((2),(1,1))}$ be the $H_{4}(0)$-module obtained by inducing the $H_{2}(0) \otimes H_{2}(0)$-module $\mathbf{C}_{(2)} \otimes \mathbf{C}_{(1,1)}$, identifying $H_{2}(0) \otimes H_{2}(0)$ to the subalgebra of $H_{4}(0)$ generated by $T_{1}$ and $T_{3}$. Let $e=1 \otimes 1$ be its standard generator, so that $T_{1} e=0, T_{3} e=-e$, and $T_{2} e$ is independent of $e$.

The automaton shown in Figure 1 gives a complete description of the induced module. Its states, which correspond to images of $e$ under the action of some basis element $T_{w}$ of $H_{4}(0)$, form a basis of $M_{((2),(1,1))}$. An arrow indexed by $T_{i}$ going from the state $f$ to $g$ means that $T_{i} \cdot f=g$, and a loop issued from a state $f$ and labelled by $T_{i} \mid \epsilon$ (with $\epsilon=0$ or $\epsilon=-1$ ) means that $T_{i} \cdot f=\epsilon f$.

This automaton is naturally graded by the distance $d(f)$ of a state $f$ to the initial state $e$ as indicated on the picture, and it is clear that this grading corresponds precisely to the filtration used in the definition of $\mathcal{F}_{q}$. To be more explicit, if one associates with each state $f$ of the automaton the composition $I(f)$ of 4 whose associated subset is

$$
D(f)=\left\{i \in[1,3] \mid T_{i} \cdot f=-f\right\}
$$

one has

$$
\mathcal{F}_{q}\left(M_{((2),(1,1))}\right)=\sum_{f} q^{d(f)} F_{I(f)}
$$


The graded characteristic of $M_{((2),(1,1))}$ is equal to

$$
\mathcal{F}_{q}\left(M_{((2),(1,1))}\right)=F_{31}+q F_{22}+q^{2}\left(F_{13}+F_{211}\right)+q^{3} F_{121}+q^{4} F_{112}
$$

For $q=1$, this characteristic is the product $F_{2} F_{11}$, which can be obtained from the shuffle of 12 and 43 [2]. On the other hand, the $q$-shuffle of 12 and 43 is

$$
12 \odot_{q} 43=1243+q 1423+q^{2} 1432+q^{2} 4123+q^{3} 4132+q^{4} 4312
$$

and taking the descent compositions of the permutations in the right-hand side, one recovers the graded characteristic of $M_{((2),(1,1))}$.

This example illustrates a general fact. The following proposition shows that the graded characteristic of an induced module as above is always given by the $q$-shuffle. As it is an associative operation, one obtains in this way a $q$-deformation of the ring of quasi-symmetric functions.

We denote here by $C(\sigma)$ the composition of $n$ associated with the descent set of a permutation $\sigma$ of $\mathfrak{S}_{n}$.

Proposition 4.15 Let $I, J$ be compositions of $n$ and $m$. Let also $\sigma$ and $\tau$ be respectively two permutations of $\mathfrak{S}_{[1, m]}$ and $\mathfrak{S}_{[m+1, m+n]}$ such that $C(\sigma)=I$ and $C(\tau)=J$. Then, the graded characteristic of the $H_{n+m}(0)$-module obtained by inducing the $H_{n}(0) \otimes H_{m}(0)$-module $\mathbf{C}_{I} \otimes \mathbf{C}_{J}$ (identifying $H_{n}(0) \otimes H_{m}(0)$ to the subalgebra of $H_{n+m}(0)$ generated by $\left.T_{1}, \ldots, T_{n-1}, T_{n+1}, \ldots, T_{n+m-1}\right)$ is given by

$$
\mathcal{F}_{q}\left(\mathbf{C}_{I} \otimes \mathbf{C}_{J} \uparrow_{H_{n}(0) \otimes H_{m}(0)}^{H_{n}+m}(0)=\sum_{\nu \in \mathbf{S}_{n+m}} q^{d(\nu)} F_{C(\nu)}\right.
$$

where one has

$$
\sigma \odot_{q} \tau=\sum_{\nu \in \varrho_{n+m}} q^{d(\nu)} \nu
$$

Proof Let $M_{I, J}$ be the induced $H_{n+m}(0)$-module considered in the proposition. This module is generated by a $e=1 \otimes 1$ on which $T_{1}, \ldots, T_{n-1}, T_{n+1}, \ldots, T_{n+m-1}$ act by

$$
T_{i} \cdot e=\left\{\begin{array}{cl}
-1 & \text { if } i \in D(I) \text { or } i \in n+D(J) \\
0 & \text { if } i \notin D(I) \text { or } i \notin n+D(J)
\end{array}\right.
$$

A basis of $M_{I, J}$ is given by elements of the standard basis $T_{\sigma}$ of $H_{n}(0)$ indexed by permutations $\sigma \in \boldsymbol{S}_{n}$ whose descent set $D(\sigma)$ is contained in $\{n\}$, i.e.

$$
\mathcal{B}_{n}=\left\{T_{\sigma} \cdot e \mid \sigma \in \mathcal{S}_{n+m} \text { and } D(\sigma) \subseteq\{n\}\right\}
$$

Let $R_{n, m}$ be the permutation

$$
R_{n, m}=m+1 m+2 \ldots m+n 12 \ldots m
$$

of $\mathfrak{S}_{n+m}$. The following lemma gives a simple characterization of the permutations indexing the elements of the basis $\mathcal{B}_{n}$ : 
Lemma 4.16 The set $\mathcal{D}_{n}=\left\{\sigma \in \mathfrak{S}_{n+m} \mid D(\sigma) \subseteq\{n\}\right\}$ is equal to the interval $\left[i d, R_{n, m}\right]$ in the permutohedron of $\mathfrak{S}_{n+m}$.

We are now in position to prove Proposition 4.15. We first suppose that $I=(n)$ and $J=(m)$. In this case, $T_{i} \cdot e=0$ when $i \neq n$. It follows that the action of the generators of $H_{n}(0)$ on the basis $\mathcal{B}_{n}$ of $M_{((n),(m))}$ is

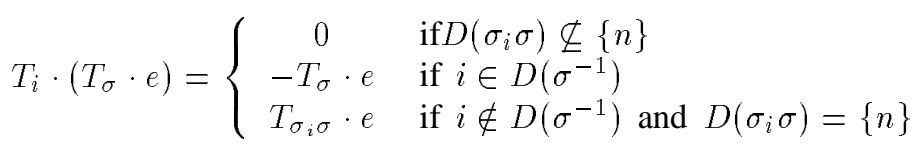

for $i \in[1, n+m-1]$ and $\sigma \in \mathcal{D}_{n}$. Let $\mathcal{J}_{n, m}$ be the linear subspace of $\mathrm{C}\left[\mathfrak{S}_{n+m}\right]$ spanned by all permutations $\sigma$ such that $D(\sigma) \nsubseteq\{n\}$, and consider the left action of $H_{n+m}(0)$ on $\mathbf{C}\left[\mathfrak{S}_{n+m}\right] / \mathcal{J}_{n, m}$ defined by

$$
T_{i} \cdot \sigma= \begin{cases}-\sigma & \text { if } \sigma(i)<\sigma(i+1) \\ \sigma \sigma_{i} & \text { if } \sigma(i)>\sigma(i+1)\end{cases}
$$

and let $\varphi_{n, m}$ be the map from $M_{((n),(m))}$ into $\mathrm{C}\left[\mathfrak{S}_{n+m}\right] / \mathcal{J}_{n, m}$ sending $T_{\sigma} \cdot e$ to the permutation $\sigma^{-1}$. Thus, $\varphi_{n, m}$ is an isomorphism between $M_{((n),(m))}$ and the $H_{n+m}(0)$-module generated by the identity permutation for the action (29). On the other hand,

$$
\sum_{\sigma \in \mathcal{D}_{n}} \sigma^{-1}=(12 \ldots n) \amalg(n+1 n+2 \ldots n+m)
$$

As an example, irı Figure 2 we show an image of the module $M_{((2),(2))}$ under $\varphi_{2,2}$.

As in Example 4.14, the graded characteristic of $M_{((n),(m))}$ can be read on the permutations indexing the states of the automaton, which are given by the formula

$$
\mathcal{F}_{q}\left(M_{((n),(m))}\right)=\mathcal{F}_{q}\left(\varphi_{n, m}\left(M_{((n),(m))}\right)\right)=\sum_{\sigma \in \mathcal{D}_{n}} q^{\ell(\sigma)} F_{C\left(\sigma^{-1}\right)}
$$

To get the proposition, it suffices therefore to prove that

$$
(12 \ldots n) \odot_{q}(n+1 n+2 \ldots n+m)=\sum_{\sigma \in \mathcal{D}_{n}} q^{\ell(\sigma)} \sigma^{-1}
$$

or equivalently that

$$
(12 \ldots n) \odot_{q}(n+1 n+2 \ldots n+m)=\sum_{\sigma \in(12 \ldots m) \uplus(n+1 n+2 \ldots n+m)} q^{\ell(\sigma)} \sigma
$$

which is clearly true. This proves therefore the proposition in special case $I=(n)$ and $J=(m)$. The general case follows from a similar argument.

Ising the formula expressing the product of two quasi-ribbon functions in terms of the shuffle product $[23,2]$ and taking $q=1$, we recover (25). 


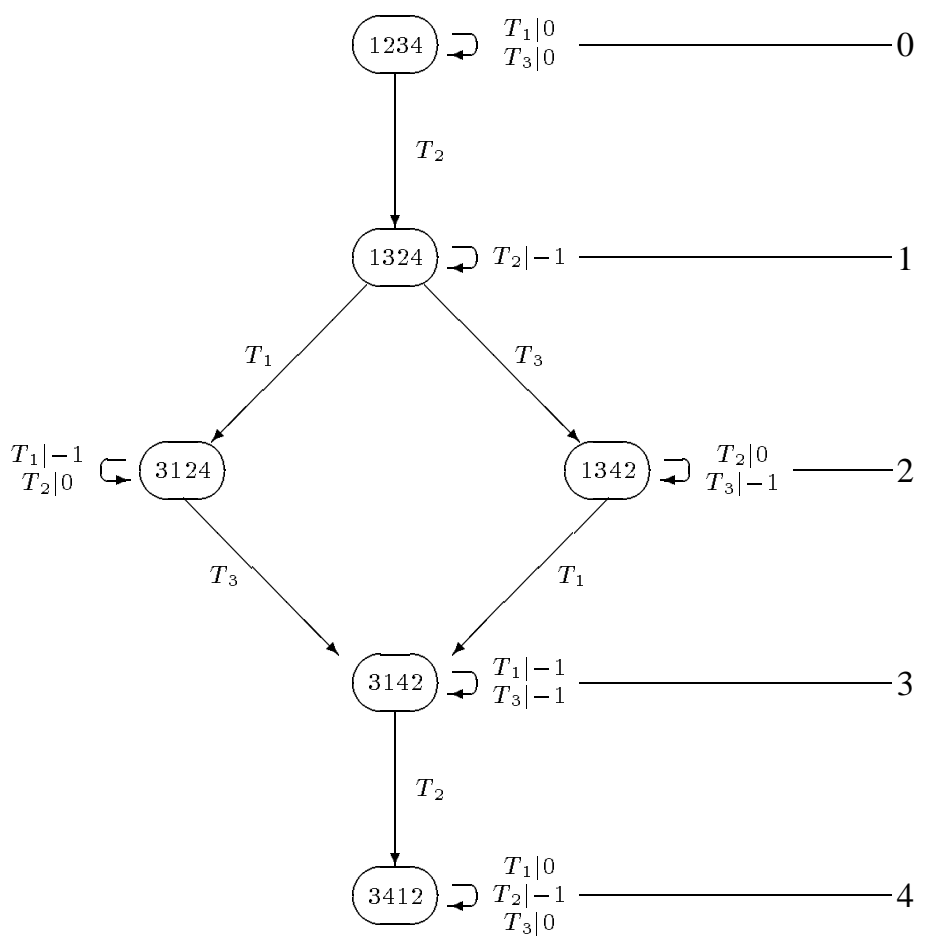

Fig. 2: The image of $M_{((2),(2))}$ under $\varphi_{2,2}$.

\section{The $q$-shuffle Hopf Algebra and its Convolution Algebra}

This section is devoted to the study of the $q$-shuffle algebra as a Hopf algebra. We describe the primitive elements, and discuss the degeneration of the convolution algebra for $q \rightarrow 1$.

\subsection{The q-shuffle Hopf Algebra}

Let $A$ be an alphabet and let $\left(K\langle A\rangle, \odot_{q}\right)$ be the corresponding $q$-shuffle algebra. As shown in Sect. 3, one can associate with it the coproduct $c_{q}$ defined by the following properties:

- $\forall a \in A, c_{q}(a)=1 \otimes a+a \otimes 1$;

- $\forall P, Q \in K\langle A\rangle, c_{q}\left(P \odot_{q} Q\right)=c_{q}(P) \odot_{q} c_{q}(Q)$.

Recall (from the proof of Proposition 3.1) that

$$
c_{q}=(U(q) \otimes U(q)) \circ c \circ U(q)^{-1}
$$

where $c=c_{0}$ is the usual coproduct of $K\langle A\rangle$. That is,

$$
c_{q}(12 \ldots n)=c\left(U_{n}(q)^{-1}\right) \cdot\left(U_{n}(q) \otimes U_{n}(q)\right)
$$


and it suffices to apply the substitution $i \rightarrow a_{i}$ to this formula to get $c_{q}\left(a_{1} a_{2} \ldots a_{n}\right)$.

Let $\omega$ be the involution sending a word $w$ to $(-1)^{|w|} \widetilde{w}$ and let $\epsilon$ be the constant term homomorphism.

Proposition $5.1\left(K\langle A\rangle, \odot_{q}, c_{q}\right)$ is a Hopf algebra with antipode $\omega$ and counit $\epsilon$.

Proof It suffices to check that $\omega$ is the antipode, with $\epsilon$ as counit. Note first that $U(q)$ and $\omega$ commute since

$$
U_{n}(q) \circ \omega_{n}=\omega_{n} \circ U_{n}(q)
$$

(where $\omega_{n}$ denotes the maximal permutation of $\mathfrak{S}_{n}$ ). The property follows then from (30), since $\omega$ is also the antipode for the usual coproduct $c$.

The combinatorial structure of the coproduct $c_{q}$ is, however, not clear at all. We tabulated $c_{q}(12 \ldots n)$ up to $n=6$, and no simple formula seems to emerge. We list below the results for $n \leq 3$.

Example 5.2 For $n=2$ and $n=3$, one has

$$
\begin{gathered}
c_{q}(12)=12 \otimes \emptyset+\frac{1}{1+q}(1 \otimes 2+2 \otimes 1)+\emptyset \otimes 12 \\
c_{q}(123)=123 \otimes \emptyset-\frac{q^{2}}{(1+q)\left(1+q+q^{2}+q^{3}+q^{4}+q^{5}\right)}(1 \otimes 32+32 \otimes 1+3 \otimes 21+21 \otimes 3) \\
+\frac{1+q^{4}}{(1+q)\left(1+q+q^{2}+q^{3}+q^{4}+q^{5}\right)}(2 \otimes 13+13 \otimes 2) \\
+\frac{q+q^{3}}{(1+q)\left(1+q+q^{2}+q^{3}+q^{4}+q^{5}\right)}(2 \otimes 31+31 \otimes 2) \\
+\frac{1+q+q^{2}+q^{3}+q^{4}}{(1+q)\left(1+q+q^{2}+q^{3}+q^{4}+q^{5}\right)}(1 \otimes 23+23 \otimes 1+12 \otimes 3+3 \otimes 12)+\emptyset \otimes 123
\end{gathered}
$$

Based on these computations, we propose the following conjecture, which would imply in particular that the convolution algebra associated with the $q$-shuffle algebra degenerates into a commutative algebra when $q \rightarrow 1$ (see Sect. 5.3).

Conjecture 5.1 There exists a family $\left(f_{\sigma, \tau}(q)\right)$ of rational functions in $\mathbf{Z}(q)$ which do not have 1 as zero nor as pole, such that for all $n \geq 1$,

$$
c_{q}(12 \ldots n)=\sum_{\substack{\sigma, \tau \\ 12 \ldots n \in \sigma \amalg \tau}} f_{\sigma, \tau}(q) \sigma \otimes \tau
$$

Note 5.3 The dual Hopf algebra of the $q$-shuffle algebra is the algebra $\left(K\langle A\rangle, \square_{q}, \Delta_{q}\right)$ with product $\square_{q}$ and coproduct $\Delta_{q}$ defined by

$$
\left(u \square_{q} v \mid w\right)=\left(u \otimes v \mid c_{q}(w)\right) \quad \text { and } \quad(\Delta(w) \mid u \otimes v)=\left(w \mid u \odot_{q} v\right)
$$


for $u, v, w \in A^{*}$. The product $\square_{q}$ dual to the coproduct $c_{q}$ is not easily described. On the other hand, one can completely describe the coproduct $\Delta_{q}$ by the following formula:

$$
\Delta_{q}(12 \ldots n)=\sum_{12 \ldots n \in \sigma \amalg \tau} q^{l(\sigma \cdot \tau)} \sigma \otimes \tau
$$

where $\sigma \cdot \tau$ denotes here the permutation obtained by concatenating $\sigma$ and $\tau$ considered as words.

\subsection{Primitive Elements for $c_{q}$}

We shall now have a look at the set $\operatorname{Prim} q$ of primitive elements for $c_{q}$. These elements occur in several decompositions of the free associative algebra and in the description of the associated convolution algebra. According to the general theory, $\operatorname{Prim}_{q}$ is just the image under $U(q)$ of the set of primitive elements for the shuffle coproduct $c$, i.e. of the free Lie algebra $L(A)$. That is,

$$
\operatorname{Prim}_{q}=U(q)(L(A))=\bigoplus_{n \geq 1} L_{n}(A) \cdot U_{n}(q)
$$

This shows that a basis of the standard component of $\operatorname{Prim}_{q}$ is, for instance, $\left(\sigma \circ \theta_{n} \circ U_{n}(q)\right)_{\sigma \in \mathcal{S}_{n}, n \geq 1}$ where $\theta_{n}$ is the Dynkin idempotent. Hence any explicit formula for $\theta_{n} \circ U_{n}(q)$ gives us an explicit description of $\operatorname{Prim}_{q}$.

To obtain a description of $\theta_{n} \circ U_{n}(q)$, let us introduce the left $t$-bracketing operator $\Theta_{n}(t)$ which is the element of $\mathbf{Z}[t]\left[\boldsymbol{S}_{n}\right]$ defined by

$$
\Theta_{n}(t)=\left[\left[\ldots\left[[1,2]_{t}, 3\right]_{t}, \ldots\right]_{t}, n\right]_{t}
$$

where $[P, Q]_{t}=P Q-t Q P$.

\section{Proposition 5.4}

$$
\Theta_{n}(t) \circ U_{n}(q)=\sum_{\sigma \in \mathcal{\Theta}_{n}} P_{\sigma}(t, q) \sigma
$$

where $P_{\sigma}(t, q)$ is the polynomial of $\mathbf{Z}[t, q]$ recursively defined by

$$
P_{1}=1 \text { and } P_{\sigma}(t, q)=\left\{\begin{array}{cl}
q^{i-1}\left(q^{n-2 i+1}-t\right) P_{\tau}(t, q) & \text { if } 1 \leq i \leq\left[\frac{n+1}{2}\right] \\
q^{n-i}\left(1-t q^{2 i-n-1}\right) P_{\tau}(t, q) & \text { if }\left[\frac{n+1}{2}\right] \leq i \leq n
\end{array}\right.
$$

where $i=\sigma^{-1}(n)$ and where $\tau$ is the permutation of $\mathfrak{S}_{n-1}$ obtained from $\sigma$ by deleting $n$.

Proof This is proved by induction using Lemma 4.4 and the well-known factorization of $\Theta_{n}(t)$ given by

$$
\Theta_{n}(t)=\left(1-t g_{2}\right)\left(1-t g_{3}\right) \ldots\left(1-t g_{n}\right)
$$

where $g_{i}$ denotes here the cycle $(i 1 \ldots i-1)$ of $\mathfrak{S}_{i}$. 
Example 5.5 Let $\sigma=23541 \in \mathfrak{S}_{5}$. Then,

$$
\begin{aligned}
P_{23541}(t, q) & =q^{2}(1-t) P_{2341}(t, q) \\
& =\left(q^{2}(1-t)\right) q(1-t q) P_{231}(t, q) \\
& =\left(q^{3}(1-t)(1-t q)\right) q(1-t) P_{21}(t, q) \\
& =\left(q^{4}(1-t)^{2}(1-t q)\right)(q-t) P_{1}(t, q) \\
& =q^{4}(q-t)(1-t)^{2}(1-t q)
\end{aligned}
$$

Specializing $t=1$ in the proposition leads to an explicit formula for $\theta_{n} \circ U_{n}(q)$. To state precisely this result, we need to introduce the elements $\tau_{n}(q)$ of $\mathbf{Z}(q)\left[\mathcal{S}_{n}\right]$ defined according to the parity of $n$ by

$$
\left\{\begin{aligned}
\tau_{2 n}(q) & =\sum_{i=1}^{n} q^{i-1} \frac{[2 n-2 i+1]_{q}}{[2 n-1]_{q}}(12 \ldots 2 n-i 2 n 2 n-i+1 \ldots 2 n-1) \\
& -\sum_{i=1}^{n} q^{n-i} \frac{[2 i-1]_{q}}{[2 n-1]_{q}}(12 \ldots n-i 2 n n-i+1 \ldots 2 n-1) \\
\tau_{2 n+1}(q) & =\sum_{i=1}^{n} q^{i-1} \frac{[2 n-2 i+2]_{q}}{[2 n]_{q}}(12 \ldots 2 n-i+12 n+12 n-i+2 \ldots 2 n) \\
& -\sum_{i=1}^{n} q^{n-i} \frac{[2 i]_{q}}{[2 n]_{q}}(12 \ldots n-i 2 n+1 n-i+1 \ldots 2 n)
\end{aligned}\right.
$$

As a consequence of Proposition 5.4, we can give a factorization formula for $\theta_{n} \circ U_{n}(q)$.

Corollary 5.6 For all $n \geq 2$,

$$
\theta_{n} \circ U_{n}(q)=(1-q)\left(1-q^{2}\right) \ldots\left(1-q^{n-1}\right) \tau_{2}(q) \tau_{3}(q) \ldots \tau_{n}(q)
$$

Example 5.7 For $n=4,5$, we have

$$
\begin{aligned}
\theta_{4} \circ U_{4}(q)= & (1-q)\left(1-q^{2}\right)\left(1-q^{3}\right)(1234-2134)(1234-3124) \\
& \left(1234+\frac{q}{1+q+q^{2}} 1243-\frac{q}{1+q+q^{2}} 1423-4123\right) \\
\theta_{5} \circ U_{5}(q)= & (1-q)\left(1-q^{2}\right)\left(1-q^{3}\right)\left(1-q^{4}\right)(12345-21345)(12345-31245) \\
& \left(12345+\frac{q}{1+q+q^{2}} 12435-\frac{q}{1+q+q^{2}} 14235-41235\right) \\
& \left(12345+\frac{q}{1+q^{2}} 12354-\frac{q}{1+q^{2}} 15234-51234\right)
\end{aligned}
$$

Let us now introduce the element $\lambda_{n}(q)$ of $\mathbf{Z}(q)\left[\mathfrak{S}_{n}\right]$ defined by

$$
\lambda_{n}(q)=[(n-1)]_{q} ! \tau_{2}(q) \tau_{3}(q) \ldots \tau_{n}(q)
$$


so that

$$
\theta_{n} \circ U_{n}(q)=(1-q)^{n-1} \lambda_{n}(q)
$$

The image of the operator $w \rightarrow w \cdot \lambda_{n}(q)$ (where $w$ runs through words of length $n$ ) is exactly the set of homogeneous primitive elements of order $n$ for $c_{q}$. In other words, one has the following description:

$$
\operatorname{Prim}_{q}=\bigoplus_{n \geq 1} K(q)<A>_{n} \cdot \lambda_{n}(q)
$$

\subsection{The Convolution Algebra}

According to the general theory, the convolution algebra $\Sigma_{n}(q)$ associated with the $q$-shuffle Hopf algebra is equal to

$$
\Sigma_{n}(q)=\bigoplus_{|I|=n} K U_{n}(q)^{-1} \circ D_{I} \circ U_{n}(q)
$$

where $D_{I}$ is the sum of all permutations with descent set $D(I)$. Unfortunately, due to the intricate structure of $U_{n}(q)$, it does not seem possible to give a simple description of $\Sigma_{n}(q)$ for $n \geq 4$ (for $n=2$ and $n=3$, one can check that $\Sigma_{n}(q)$ is in fact equal to the usual descent algebra $\Sigma_{n}$ ).

The following conjecture, basically due to Zagier [25], would give some indications on the structure of $U_{n}(q)^{-1}$ :

Conjecture $5.2{ }^{\dagger}$ Let $\omega_{n}$ denote the maximal permutation of $\mathfrak{S}_{n}$. For all $n \geq 1$, the inverse of $U_{n}(q)$ has the form

$$
U_{n}(q)^{-1}=\frac{1}{\left(1-q^{2}\right) \ldots\left(1-q^{n(n-1)}\right)}\left(\sum_{\sigma \in \mathcal{D}_{n}}(-1)^{|D(\sigma)|} p_{\sigma}(q) \sigma\right)\left(1+(-1)^{n-1} q^{n(n-1) / 2} \omega_{n}\right)
$$

where $p_{\sigma}(q)$ is a polynomial in $\mathbf{N}[q]$ and where $\mathcal{D}_{n}$ is a subset of $\boldsymbol{S}_{n}$ whose cardinality is equal to the number of planar trees with n leaves.

Zagier's conjecture would give a description of the limit of the convolution algebra $\Sigma_{n}(q)$ when $q \rightarrow 1$ whenever this degenerate limit exists ${ }^{\ddagger}$. Indeed, a basis of $\Sigma_{n}(q)$ is given by

$$
\alpha_{q}\left(\Psi^{I}\right)=\left(U_{i_{1}}(q)^{-1} \circ \theta_{i_{1}} \circ U_{i_{1}}(q)\right) \otimes_{q} \cdots \otimes_{q}\left(U_{i_{r}}(q)^{-1} \circ \theta_{i_{r}} \circ U_{i_{r}}(q)\right)
$$

for $|I|=n$. The conjecture would imply, taking into account (34), that

$$
\lim _{q \rightarrow 1} U_{n}(q)^{-1} \circ \theta_{n} \circ U_{n}(q)=\frac{1}{n !}\left(\sum_{\sigma \in \mathcal{D}_{n}}(-1)^{|D(\sigma)|} p_{\sigma}(1) \sigma\right)\left(1+(-1)^{n-1} \omega_{n}\right) \lambda_{n}(1)
$$

These elements would therefore generate the limit convolution algebra $\Sigma_{n}(1)$, if it exists.

$\dagger$ Added in proof: This conjecture has been recently disproved by Melianac and Svrtan [17], who found a counterexample for $n=8$. These authors also give a precise description of $U_{n}(q)^{-1}$.

$\ddagger$ The existence of a limit convolution algebra $\Sigma_{n}$ (1) would be a consequence of Conjecture 5.1. 
The expression of $U_{n}(q)^{-1}$ given by Zagier's Conjecture can be interpreted as the decomposition on the family $\left(\sigma \lambda_{n}(1)\right)$ of the idempotent corresponding to the projection on the homogeneous component of order $n$ of

$$
\mathcal{L}=\bigoplus_{n \geq 1} K\langle A\rangle_{n} \cdot \lambda_{n}(1)
$$

with respect to the decomposition

$$
K\langle A\rangle=K \oplus \mathcal{L} \oplus \mathcal{L} \omega \mathcal{L} \oplus \cdots \oplus \underbrace{\mathcal{L} \omega \ldots \amalg \mathcal{L}}_{\mathrm{n} \text { times }} \oplus \ldots
$$

of the free associative algebra $K\langle A\rangle$.

\section{Some Generalizations of the Shuffle Operator}

The aim of this section is to present interesting generalizations of the shuffle operator of which the $q$ shuffle product is the simplest case.

\subsection{Rosso's Quantum Shuffles}

Rosso showed, by expliciting their multiplication, that certain Hopf algebras obtained by very general constructions can be interpreted as generalizations of the shuffle algebra [24]. These algebras are constructed as follows.

Let $H$ be a Hopf algebra over a field $K$. A Hopf bimodule $M$ over $H$ is a $K$-vector space endowed with compatible structures of $H$-bimodule and $H$-bicomodule [29]. In other words, a Hopf bimodule over $H$ is a $H$-bimodule $M$ equipped with left and right coactions $\delta_{L}: M \rightarrow H \otimes M$ and $\delta_{R}: M \rightarrow M \otimes H$ commuting with each other (i.e. such that $\left.\left(i d \otimes \delta_{R}\right) \delta_{L}=\left(\delta_{L} \otimes i d\right) \delta_{R}\right)$ and which are morphisms of $H$-bimodules.

The following proposition is a particular case of a result of Woronowicz:

Proposition 6.1 [28] Let $M$ be a Hopf H-bimodule. There exists a unique H-bimodule endomorphism $\sigma_{M}$ of $M \otimes_{H} M$ such that

$$
\forall m, n \in M, \delta_{L}(m)=1 \otimes m, \delta_{R}(n)=n \otimes 1 \quad \Longrightarrow \quad \sigma_{M}(m \otimes n)=n \otimes m
$$

Moreover, $\sigma_{M}$ is invertible and satisfies to the braid equation

$$
\left(I d_{M} \otimes \sigma_{M}\right)\left(\sigma_{M} \otimes I d_{M}\right)\left(I d_{M} \otimes \sigma_{M}\right)=\left(\sigma_{M} \otimes I d_{M}\right)\left(I d_{M} \otimes \sigma_{M}\right)\left(\sigma_{M} \otimes I d_{M}\right)
$$

Recall that the braid group $\mathcal{B}_{n}$ is the (infinite) group generated by $n-1$ elements $\left(s_{i}\right)_{i=1, n-1}$ subject to the relations

$$
\begin{array}{cl}
s_{i} s_{j}=s_{j} s_{i} & \text { for }|i-j|>1 \\
s_{i} s_{i+1} s_{i}=s_{i+1} s_{i} s_{i+1} & \text { for } i \in[1, n-2]
\end{array}
$$

For each permutation $\sigma \in \mathcal{S}_{n}$, one can define an element $T_{\sigma}$ of $\mathcal{B}_{n}$ by $T_{\sigma}=s_{i_{1}} \ldots s_{i_{r}}$ where $\sigma_{i_{1}} \ldots \sigma_{i_{r}}$ is an arbitrary reduced decomposition of $\sigma$. 
Let $M$ be a Hopf $H$-bimodule and let $M^{L}=\left\{m \in M, \delta_{L}(m)=1 \otimes m\right\}$ be the submodule of left coinvariants. The proposition allows us to define a right action of the braid group $\mathcal{B}_{n}$ on $\left(M^{L}\right)^{\otimes n}$ by

$$
x_{1} \otimes \cdots \otimes x_{n} \cdot s_{i}=\left(I d_{M^{L}}^{\otimes(i-1)} \otimes \sigma_{M} \otimes I d_{M^{L}}^{\otimes(n-i-1)}\right)\left(x_{1} \otimes \cdots \otimes x_{n}\right)
$$

for every $x_{1}, \ldots, x_{n} \in M^{L}$.

On the other hand, it can be shown that $T\left(M^{L}\right)$ is isomornhic to the snace of left coinvariants of the cotensor coalgebra $T_{H}^{c}(M)$, which is known by a result of Nichols [29] to be a Hopf algebra. This isomorphism therefore endows $T\left(M^{L}\right)$ with a new product $\odot$, and Rosso shows that it can be explicited as follows, in terms of the braid group action on $T\left(M^{L}\right)$ :

\section{Proposition 6.2}

$$
\left(x_{1} \otimes \cdots \otimes x_{p}\right) \odot\left(x_{p+1} \otimes \cdots \otimes x_{n}\right)=\sum_{\sigma \in 12 \ldots p \amalg p+1 p+2 \ldots n}\left(x_{1} \otimes \cdots \otimes x_{n}\right) \cdot T_{\sigma}
$$

where $\cdot$ denotes the right action of $\mathcal{B}_{n}$ on $\left(M^{L}\right)^{\otimes n}$ defined by (35).

For our purposes, one can consider the following reversed presentation. Let $V$ be any vector space, and $R \in$ End $(V \otimes V)$ be a solution of the Yang-Baxter equation

$$
R_{12} R_{13} R_{23}=R_{23} R_{13} R_{12}
$$

where as usual $R_{i, j}$ is the endomorphism of $V \otimes V \otimes V$ acting as $R$ on the $i$-th and $j$-th factors. Also, let $\breve{R}=P R$, where $P(u \otimes v)=v \otimes u$, so that $\breve{R}$ satisfies the braid relation

$$
\breve{R}_{12} \breve{R}_{23} \breve{R}_{12}=\breve{R}_{23} \check{R}_{12} \breve{R}_{23}
$$

One defines then as usual a right action of $\mathcal{B}_{n}$ on $V^{\otimes n}$ by

$$
v_{1} \otimes v_{2} \otimes \cdots \otimes v_{n} \cdot s_{i}=\check{R}_{i, i+1}\left(v_{1} \otimes v_{2} \otimes \cdots \otimes v_{n}\right)
$$

The fundamental observation (also known tc Rosso [30]; is the following

Lemma 6.3 Define a multiplication $\odot$ on $T(V)$ by

$$
\left(x_{1} \otimes \cdots \otimes x_{p}\right) \odot\left(x_{p+1} \otimes \cdots \otimes x_{n}\right)=\sum_{\sigma \in 12 \ldots p \amalg p+1 p+2 \ldots n}\left(x_{1} \otimes \cdots \otimes x_{n}\right) \cdot T_{\sigma}
$$

for $x_{1}, \ldots, x_{n} \in V$. Then, $\odot$ is associative.

Proof Consider first the case where $R$ is the identity of $V \otimes V$. Then $\odot$ is the ordinary shuffle product, which is indeed associative. Let $\rho$ be the representation of $\mathcal{B}_{\infty}$ defined by $R$, and let

$$
\operatorname{sh}(p, q)=\sum_{\sigma \in 12 \ldots p} T_{\sigma}
$$

The associativity of $\odot$ is equivalent to the validity of the identities

$$
\rho(\operatorname{sh}(p, q) \operatorname{sh}(p+q, r))=\rho(\operatorname{sh}(q, r) \operatorname{sh}(p, q+r))
$$


in the given representation. But taking reduced decompositions of both sides and expanding the products, one obtains only reduced words. The identity being true for the symmetric group, it is valid in the algebra of the braid group, and therefore in any representation.

Example 6.4 (1) The $q$-shuffle $\odot_{q}$ is the particular case obtained by taking $R=q \cdot I$, where $I$ is the identity of $V \otimes V$ (and $V$ is $\bigoplus_{a \in A} K a$ ).

(2) Let $\left(e_{i}\right)$ be a basis of $V$. Diagonal matrices $R\left(e_{i} \otimes e_{j}\right)=q_{i j} e_{i} \otimes e_{j}$ satisfy the Yang-Baxter equation. If $A=\left(a_{i j}\right)$ is a symmetrizable Cartan matrix and if $\left(d_{i}\right)$ are relatively prime positive integers such that $\left(d_{i} a_{i j}\right)$ is symmetric, Rosso shows that the triangular part of the quantized enveloping algebra associated to $A$ is a subalgebra of the corresponding quantum shuffle algebra.

(3) Take $V=K[x]$, where $K=\mathrm{C}(q)$, and identify $V^{\otimes n}$ with $K\left[x_{1}, x_{2}, \ldots, x_{n}\right]$. The standard action of the Hecke algebra $H_{n}(q)$ on $K\left[x_{1}, \ldots, x_{n}\right]$ by symmetrizing operator; [31]

$$
T_{i}=(q-1) \pi_{i}+\sigma_{i}
$$

where $\pi_{i}$ is the isobaric divided difference operator

$$
\pi_{i}(P)=\frac{x_{i} P-x_{i+1} \sigma_{i}(P)}{x_{i}-x_{i+1}}
$$

gives a solution of the Yang-Baxter equation and induces as above an action of the braid group on $V^{\otimes n}$. The subspace of $V^{\otimes n}$ spanned by $\odot$ products of elements of $V$ can be identified with the space of symmetric polynomials in $x_{1}, \ldots, x_{n}$, and the factorization formula for the total symmetrizer $S^{(n)}=\sum_{\sigma \in \mathfrak{S}_{n}} T_{\sigma}$ given in [31], Theorem 3.1, implies that for a partition $\lambda=\left(\lambda_{1} \geq \lambda_{2} \geq \ldots \geq \lambda_{n}>0\right)$, the product

$$
x^{\lambda_{1}} \odot x^{\lambda_{2}} \odot \cdots \odot x^{\lambda_{n}}
$$

is equal, up to a scalar factor, to the Hall-Littlewood polynomial $Q_{\lambda}\left(x_{1}, \ldots, x_{n} ; 1 / q\right)$.

(4) Other families of symmetric functions can be obtained by considering degenerate actions, for example, the Schur functions or the augmented monomial functions

(5) The $q$-wedge product introduced by Stern [32] and Kashiwara-Miwa-Stern [16] can also be regarded as a special case of this construction, obtained from an action of the affine Hecke aigebra commuting with $U_{q}\left(\hat{s l}_{n}\right)$.

\subsection{Twisted Derivations and Multi-parameter Deformations of the Shuffle Prod- uct}

This section explains how the consideration of twisted derivations allows one to recover in a natural way the multi-parameter deformations of the shuffle product associated to diagonal solutions of the YangBaxter equation.

Definition 6.5 A twisted derivation for a $K$-algebra structure $(K\langle A\rangle,+, \odot)$ constructed over $K\langle A\rangle$ is a linear map $\partial$ from $K\langle A\rangle$ into $K\langle A\rangle$ which satisfies for words $u, v \in A^{*}$ to the Leibnitz rule

$$
\partial(u \odot v)=\partial(u) \odot v+\chi(u) u \odot \partial(v)
$$

where $\chi$ is a monoid morphism from $A^{*}$ into $K$. 
Example 6.6 Let $\left(q_{a}\right)_{a \in A}$ (resp. $\left.(\partial(a))_{a \in A}\right)$ be an arbitrary family of elements of $K$ (resp. of $K\langle A\rangle$ ). One can define a derivation $\partial$ on the free associative algebra $(K\langle A\rangle,+, \cdot)$ by

$$
\partial(w)=\sum_{u a v=w} q_{u} u \partial(a) v
$$

where for $u=a_{1} \cdots a_{n} \in A^{*}, q_{u}$ denotes the element $q_{a_{1}} \ldots q_{a_{n}}$.

Consider now the classical twisted derivations $\left(\partial_{a}\right)_{a \in A}$ on $K\langle A\rangle$, defined for each letter $a \in A$ by

$$
\partial_{a}(w)= \begin{cases}u & \text { if } w=a u \\ 0 & \text { if } w \notin a A^{*}\end{cases}
$$

for $w \in A^{*}$. These operators are widely used in automata theory, e.g. for defining the algebra of rational series (cf. [33]). They are also twisted derivations for the $q$-shuffle algebra, since by definition

$$
\partial_{a}\left(u \odot_{q} v\right)=\partial_{a}(u) \odot_{q} v+q^{|u|} u \odot_{q} \partial_{a}(v)
$$

for $u, v \in A^{*}$. One can then ask whether there are other products for which all operators $\partial_{a}$ are still twisted derivations. The following proposition answers this question in the case where the twisting morphism $\chi$ is given by means of a matrix of commutation factors.

Proposition 6.7 Let $M=\left(q_{a, b}\right)_{a, b \in A}$ be an $A \times A$-matrix of indeterminates, and set

$$
q(u, v)=\prod_{i, j} q_{a_{i}, b_{j}}
$$

for all words $u=a_{1} \ldots a_{n}$ and $v=b_{1} \ldots b_{m}$. There exists then a unique graded product $\odot{ }_{M}$ on $K\langle A\rangle$ such that

$$
\begin{gathered}
\forall u \in A^{*}, u \odot_{M} 1=1 \odot_{M} u=u \\
\forall u, v \in A^{*}, \partial_{a}\left(u \odot_{M} v\right)=\partial_{a}(u) \odot_{M} v+q(a, u) u \odot_{M} \partial_{a}(v)
\end{gathered}
$$

for every $a \in A$. This product can be computed by the following recursive formulas

$$
\begin{gathered}
u \odot_{M} 1=1 \odot_{M} u=u \\
(a u) \odot_{M}(b v)=a\left(u \odot_{M} b v\right)+q(b, a u) b\left(a u \odot_{M} v\right)
\end{gathered}
$$

where $u, v \in A^{*}$ and $a, b \in A$.

Proof A straightforward calculation, which is left to the reader.

The product $\odot_{M}$ defined in the proposition is associative. One can therefore consider the algebra $\left(K\langle A\rangle,+, \odot_{M}\right)$. Observe that this algebra reduces to the $q$-shuffle algebra when all indeterminates $q_{a, b}$ are equal to $q$. In the general case, the product $\odot_{M}$ is still a special case of the construction of Sect. 3 . Indeed,

$$
a_{1} \odot_{M} a_{2} \odot_{M} \cdots \odot_{M} a_{n}=a_{1} a_{2} \ldots a_{n} \cdot U_{n}(M)
$$


for $a_{i} \in A$, and where

$$
U_{n}(M)=\sum_{\sigma \in \mathfrak{S}_{n}}\left(\prod_{\substack{1 \leq i<j \leq n \\ \sigma(i)>\sigma(j)}} q_{a_{\sigma(i)}, a_{\sigma(j)}}\right) \sigma
$$

Thus, $\left(K\langle A\rangle,+, \odot_{M}\right)$ is shuffle Hopf algebra associated to the diagonal solution $R(x \otimes y)=q_{x, y}(x \otimes y)$ of the Yang-Baxter equation.

Note 6.8 If one forgets about the first condition in the proposition, there are other interesting solutions, such as the $T$-operation of Schützenberger [34], also called 'chronological product' [35].

Note 6.9 When the matrix $M$ is skew-symmetric in the multiplicative sense, i.e. when $q_{a, b}=q_{b, a}^{-1}$ for every $a, b \in A$, one recovers the bicharacters introduced by Ree [10]. l_et us recall that a bicharacter $\chi$ is a bilinear mapping from $\mathbf{N}^{(A)} \times \mathbf{N}^{(A)}$ into $(K, \times)$, i.e. a mapping such that

$$
\chi(x, y+z)=\chi(x, y) \chi(x, z) \quad \text { and } \quad \chi(x+y, z)=\chi(x, z) \chi(y, z)
$$

hold for every $x, y, z \in \mathbf{N}^{(A)}$. Let now $\left(\varepsilon_{a}\right)_{a \in A}$ be the basis of $\mathbf{N}^{(A)}$ defined by $\varepsilon_{a}(b)=\delta_{a, b}$ for $a, b \in A$. The mapping which associates to a skew-symmetric matrix $M=\left(q_{a, b}\right)_{a, b \in A}$ the bicharacter $\chi\left(\varepsilon_{a}, \varepsilon_{b}\right)=q_{a, b}$ is then clearly a one-to-one correspondence between bicharacters and skew-symmetric $A \times A$-matrices.

It is also interesting to observe that End ${ }^{g r}(K\langle A\rangle)$ can be equipped in this case with a structure of Lie superalgebra defined by

$$
[f, g]=f g-\chi(\operatorname{deg}(f), \operatorname{deg}(g)) g f
$$

The space of graded twisted derivations over $\left(K\langle A\rangle,+, \odot_{M}\right)$ becomes then a super Lie subalgebra of this superalgebra. Moreover it can be shown that the derivations $\left(\partial_{a}\right)_{a \in A}$ generates the free Lie superalgebra $L_{\chi}(A)$.

\subsection{Shuffle Operators in the Braid Group Algebra}

Rosso's construction suggests that the combinatorics of generalized shuffles has to be uderstood at the level of the braid group algebra. This point of view leads to a uniform presentation of several factorization results, and reveals a connection between quantum shuffles and Varchenko's construction of a quantum bilinear form associated to a hyperplane arrangement.

\subsubsection{A Shuffle Element in the Braid Group Algebra}

Let $\mathcal{B}_{\infty}$ denote the infinite braid group, generated by elements $\left(s_{i}\right)_{i>1}$ satisfying to the relations $s_{i} s_{j}=$ $s_{j} s_{i}$ for $|i-j|>1$ and $s_{i} s_{i+1} s_{i}=s_{i+1} s_{i} s_{i+1}$. Since every braid group $\mathcal{B}_{n}$ can be embedded in $\mathcal{B}_{\infty}$, the notation $T_{\sigma}$ will be still used in this context.

One can define a shuffle element $\mathcal{U}_{n}$ in $K\left[\mathcal{B}_{\infty}\right]$ by

$$
\mathcal{U}_{n}=\sum_{\sigma \in \mathfrak{S}_{n}} T_{\sigma}
$$

The $q$-shuffle operator is the image of $\mathcal{U}_{n}$ under the representation $s_{i} \rightarrow q \sigma_{i}$. Thus any factorization of $\mathcal{U}_{n}$ will also hold in particular for the $q$-shuffle operator $U_{n}(q)$. 


\subsubsection{Factorizations}

More generally, any factorization of $\mathcal{U}_{n}$ will provide similar decompositions in any homomorphic image of the braid group. We will first give a decomposition formula for $\mathcal{U}_{n}$ which can be viewed as the generalization of Zagier's factorization of the $q$-shuffle operator.

Consider the element $\mathcal{T}_{n}$ of the algebra of $\mathcal{B}_{\infty}$ defined by

$$
\mathcal{T}_{n}=1+s_{n-1}+s_{n-1} s_{n-2}+\cdots+\left(s_{n-1} s_{n-2} \ldots s_{1}\right)
$$

One can then give the following factorization of $\mathcal{U}_{n}$ in $\mathrm{N}\left[\mathcal{B}_{\infty}\right]$ :

Proposition 6.10 For every $n \geq 2$, one has

$$
\mathcal{U}_{n}=\mathcal{T}_{2} \mathcal{T}_{3} \ldots \mathcal{T}_{n}
$$

Proof The formula is true in the representation $s_{i} \mapsto \sigma_{i}$, and expanding the product yields only reduced words.

The elements $\mathcal{T}_{n}$ can themselves be factorized, and we obtain in this way a factorization of $\mathcal{U}_{n}$ in $\mathrm{Z}\left[\left[\mathcal{B}_{\infty}\right]\right]$, which projects onto Zagier's factorization under $s_{i} \rightarrow q \sigma_{i}$.

Proposition 6.11 For $n \geq 2$, one has in $\mathbf{Z}\left[\left[\mathcal{B}_{\infty}\right]\right]$ :

$$
\begin{aligned}
\mathcal{T}_{n}= & \left(1-s_{n-1}^{2} s_{n-2} \ldots s_{1}\right)\left(1-s_{n-1}^{2} s_{n-2} \ldots s_{2}\right) \ldots\left(1-s_{n-1}^{2}\right) \\
& \left(1-s_{n-1}\right)^{-1}\left(1-s_{n-1} s_{n-2}\right)^{-1} \ldots\left(1-s_{n-1} s_{n-2} \ldots s_{1}\right)^{-1}
\end{aligned}
$$

Proof The proposition follows from the following lemma, whose proof is left to the reader.

Lemma 6.12 For $n \geq 3$, one has

$$
\mathcal{T}_{n}\left(1-s_{n-1} s_{n-2} \ldots s_{1}\right)=\left(1-s_{n-1}^{2} s_{n-2} \ldots s_{1}\right) t_{n-1}
$$

where $t_{n-1}$ denotes the image of $\mathcal{T}_{n-1}$ under the algebra morphism $\eta$ of $\mathbf{N}\left[\mathcal{B}_{\infty}\right]$ defined by $\eta\left(s_{i}\right)=s_{i+1}$ for all $i \geq 1$.

Example 6.13 For $n=2,3,4$,

$$
\begin{gathered}
\mathcal{T}_{2}=\left(1-s_{1}^{2}\right)\left(1-s_{1}\right) \\
\mathcal{T}_{3}=\left(1-s_{2}^{2} s_{1}\right)\left(1-s_{2}^{2}\right)\left(1-s_{2}\right)^{-1}\left(1-s_{2} s_{1}\right)^{-1} \\
\mathcal{T}_{4}=\left(1-s_{3}^{2} s_{2} s_{1}\right)\left(1-s_{3}^{2} s_{2}\right)\left(1-s_{3}^{2}\right)\left(1-s_{3}\right)^{-1}\left(1-s_{3} s_{2}\right)^{-1}\left(1-s_{3} s_{2} s_{1}\right)^{-1}
\end{gathered}
$$

Specializing these results in a homorphic image of the braid group, we get the following useful corollary:

Corollary 6.14 For any representation $\rho$ of $\mathcal{B}_{n}$, one has

$$
\rho\left(\mathcal{U}_{n}\right)=\rho\left(\mathcal{T}_{2}\right) \rho\left(\mathcal{T}_{3}\right) \ldots \rho\left(\mathcal{T}_{n}\right)
$$

and if the inverses are defined,

$$
\rho\left(\mathcal{T}_{n}\right)=\prod_{1 \leq i \leq n-1}^{\overrightarrow{1}}\left(1-\rho\left(s_{n-1}^{2} s_{n-2} \ldots s_{i}\right)\right) \prod_{1 \leq i \leq n-1}^{\leftarrow}\left(1-\rho\left(s_{n-1} s_{n-2} \ldots s_{i}\right)\right)^{-1}
$$


Note 6.15 Typical examples where the inverses are well defined are the representations $s_{i} \longrightarrow q s_{i}$ in $\mathrm{C}\left[\mathfrak{S}_{n}\right]$ or the Hecke algebra $H_{n}(t)$ where the factors of the elements $\rho\left(\mathcal{T}_{i}\right)$ satisfy polynomial equations with non-zero constant terms and hence are invertible. For example for the symmetric group, with $X=$ $\rho\left(s_{n-1} s_{n-2} \ldots s_{i}\right)\left(\right.$ resp. $\left.Y=\rho\left(s_{n-1}^{2} s_{n-2} \ldots s_{i}\right)\right)$ one has

$$
X^{n-i+1}=q^{(n-i+1)(n-i)} ;(1-X)\left(\sum_{j=0}^{n-i} X^{j}\right)=1-q^{(n-i+1)(n-i)}
$$

Hence

$$
(1-X)^{-1}=\left(1-q^{(n-i+1)(n-i)}\right)^{-1}\left(\sum_{j=0}^{n-i} X^{j}\right)
$$

similarly

$$
Y^{n-i-1}=q^{(n-i+1)(n-i)} ;(1-Y)\left(\sum_{j=0}^{n-i-2} Y^{j}\right)=1-q^{(n-i+1)(n-i)}
$$

and then

$$
(1-Y)^{-1}=\left(1-q^{(n-i+1)(n-i)}\right)^{-1}\left(\sum_{j=0}^{n-i-2} Y^{j}\right)
$$

so that the denominator of Zagier's formula can be lowered to

$$
\prod_{m=2}^{n} \prod_{i=1}^{m-1}\left(1-q^{(m-i+1)(m-i)}\right)=\prod_{k=1}^{n-1}\left(1-q^{k(k+1)}\right)^{n-k}
$$

\subsubsection{Action of the Braid Group on Words}

Let $M=\left(q_{a, b}\right)_{a, b \in A}$ be a matrix of indeterminates. The diagonal solutions to the Yang-Baxter equation discussed in Example 6.4 can be described as the right action $\cdot$ of $\mathcal{B}_{n}$ on words defined by

$$
\left(a_{1} a_{2} \ldots a_{n}\right) \cdot s_{i}=q_{a_{i}, a_{i+1}}\left(a_{1} \ldots a_{i-1} a_{i+1} a_{i} a_{i+2} \ldots a_{n}\right)
$$

for $a_{i} \in A$.

Example 6.16 The matrices of $\mathcal{T}_{2}$ and $\mathcal{T}_{3}$ considered as operators on the space $K a_{1} a_{1} a_{2} \oplus K a_{1} a_{2} a_{1} \oplus$ $K a_{2} a_{1} a_{1}$ with respect to this action are given by

$$
\begin{gathered}
\mathcal{T}_{2}=\left[\begin{array}{ccc}
1+q_{a_{1}, a_{1}} & 0 & 0 \\
0 & 1 & q_{a_{1}, a_{2}} \\
0 & q_{a_{2}, a_{1}} & 1
\end{array}\right] \\
\mathcal{T}_{3}=\left[\begin{array}{ccc}
1 & q_{a_{1}, a_{2}} & q_{a_{1}, a_{2}}^{2} \\
q_{a_{2}, a_{1}}+q_{a_{1}, a_{1}} q_{a_{2}, a_{1}} & 1 & 0 \\
0 & q_{a_{2}, a_{1}} q_{a_{1}, a_{1}} & 1+q_{a_{1}, a_{1}}
\end{array}\right]
\end{gathered}
$$


This subsection will be devoted to the study of the determinant of $\mathcal{U}_{n}$ considered as an operator acting on words of fixed multihomogeneity. According to the results of Sect. 6.3.2, one can compute this determinant if one knows the values of

$$
\operatorname{det}\left(1-s_{n}^{2} s_{n-1} \ldots s_{m}\right) \text { and } \operatorname{det}\left(1-s_{n} s_{n-1} \ldots s_{m}\right)
$$

for all $m<n$. But for the representation under consideration,

$$
\begin{aligned}
& \operatorname{det}\left(1-s_{n}^{2} s_{n-1} \ldots s_{m}\right)=\operatorname{det}\left(1-s_{n-m+1}^{2} s_{n-m} \ldots s_{1}\right) \\
& \operatorname{det}\left(1-s_{n} s_{n-1} \ldots s_{m}\right)=\operatorname{det}\left(1-s_{n-m+1} s_{n-m} \ldots s_{1}\right)
\end{aligned}
$$

for $1 \leq m \leq n-1$. Hence it is sufficient to compute the determinants of $1-s_{n-1} s_{n-2} \ldots s_{1}$ and $1-s_{n-1}^{2} s_{n-2} \ldots s_{1}$. The following proposition gives an answer to this question by providing explicit formulas for the characteristic polynomials det $(1-x s)$ of the elements $s=s_{n} s_{n-1} \ldots s_{1}$ and $s=$ $s_{n}^{2} s_{n-1} \ldots s_{1}$.

For $I \in \mathbf{N}^{(A)}$, let $K\langle A\rangle_{I}$ be the multihomogenous component of multidegree $I$ of $K\langle A\rangle$. Every braid $s \in \mathcal{B}_{|I|-1}$ stabilizes $K\langle A\rangle_{I}$. We can therefore consider $s$ as an operator of $K\langle A\rangle_{I}$. The characteristic polynomial of this operator will be denoted by $P_{I}(s ; x)$. Finally, let us set

$$
q(I, J)=\prod_{a, b \in A} q_{a, b}^{I_{a} \times J_{b}} \quad \text { and } \quad q(I)=\prod_{a \in A} q_{a, a}^{I_{a}}
$$

for $I=\left(I_{a}\right)_{a \in A}$ and $J=\left(J_{a}\right)_{a \in A}$ of $\mathbf{N}^{(A)}$.

Proposition 6.17 For $n \geq 1$ and $I \in \mathbf{N}^{(A)}$ of weight $|I|=n$, one has

$$
\begin{gathered}
P_{I}\left(s_{n-1} s_{n-2} \ldots s_{1} ; x\right)=\prod_{\substack{\left.I=k J \\
k \in \mathrm{N} \\
k \in s_{1} ; x\right)}}\left(x^{|J|}-\frac{q(J, J)^{k}}{q(J)}\right)^{l_{J}} \\
P_{I}\left(s_{n-1}^{2} s_{n-2} \ldots s_{\substack{I=k J+\varepsilon_{a} \\
k \in \mathrm{N}, a \in A}}\left(x^{|J|}-\frac{q(J, J)^{k} q\left(J, \varepsilon_{a}\right) q\left(\varepsilon_{a}, J\right)}{q(J)}\right)^{l_{J}}\right.
\end{gathered}
$$

where $\left(\varepsilon_{a}\right)=\delta_{a, b}$ and where $l_{J}$ denotes the dimension of the multihomogeneous component of multihomogeneity $J$ of the free Lie algebra $L(A)$.

Proof These formulas are obtained by expliciting the eigenvectors of the powers of $s$.

Example 6.18 Consider the multidegree $I=(2,2,0,0, \ldots)$. In this case, the matrix of the operator $s_{3} s_{2} s_{1}$ in the basis $\left\{a_{1} a_{1} a_{2} a_{2}, a_{1} a_{2} a_{1} a_{2}, a_{1} a_{2} a_{2} a_{1}, a_{2} a_{1} a_{1} a_{2}, a_{2} a_{1} a_{2} a_{1}, a_{2} a_{2} a_{1} a_{1}\right\}$ of $K\langle A\rangle_{I}$ is

$$
M_{s_{3} s_{2} s_{1}}=\left(\begin{array}{cccccc}
0 & 0 & q_{11} q_{21}^{2} & 0 & 0 & 0 \\
0 & 0 & 0 & 0 & q_{11} q_{21}^{2} & 0 \\
0 & 0 & 0 & 0 & 0 & q_{11} q_{21}^{2} \\
q_{12}^{2} q_{22} & 0 & 0 & 0 & 0 & 0 \\
0 & q_{12}^{2} q_{22} & 0 & 0 & 0 & 0 \\
0 & 0 & 0 & q_{12}^{2} q_{22} & 0 & 0
\end{array}\right)
$$


where we write for convenience $q_{i, j}$ in place of $q_{a_{i}, a_{j}}$. Then,

$$
P_{I}\left(s_{3} s_{2} s_{1} ; x\right)=\left(x^{4}-q_{21}^{4} q_{12}^{4} q_{11}^{2} q_{22}^{2}\right)\left(x^{2}-q_{21}^{2} q_{12}^{2} q_{11} q_{22}\right)
$$

according to the first formula of the proposition.

As an illustration, we can give an expression of the characteristic polynomial of the permutation $\sigma_{n-1} \ldots \sigma_{1}$ in an irreducible representation $V_{\lambda}$ of $\mathfrak{S}_{n}$.

Corollary 6.19 Let $\lambda$ be a partition of $n$. The characteristic polynomial of the permutation $\sigma_{n-1} \ldots \sigma_{1}$ in the irreducible representation $V_{\lambda}$ of $\mathfrak{S}_{n}$ is

$$
P_{V_{\lambda}}\left(\sigma_{n-1} \ldots \sigma_{1} ; x\right)=\prod_{\mu}\left(\prod_{\lambda=k I, k \in \mathrm{N}}\left(x^{|I|}-1\right)^{l_{I}}\right)^{\left\langle s_{\lambda}, m_{\mu}\right\rangle}
$$

where $s_{\lambda}=\sum_{\mu}\left\langle s_{\lambda}, m_{\mu}\right\rangle h_{\mu}$ denotes the decomposition of the Schur function $s_{\lambda}$ on the basis of complete symmetric functions.

Proof For a $K\left[\mathfrak{S}_{n}\right]$-module $V$ and for $s \in \mathfrak{S}_{n}$, denote by $P_{V}(s ; x)$ the characteristic polynomial of $s$ in the representation $V$. The corollary follows from the property

$$
P_{V+W}(s ; x)=P_{V}(s ; x) P_{W}(s ; x)
$$

and from the fact that the characteristic polynomial of $\sigma_{n-1} \ldots \sigma_{1}$ in the permutation representation $S^{\lambda}$ of $\mathfrak{S}_{n}$ whose Frobenius characteristic is $h_{\lambda}$ is given by

$$
P_{S^{\lambda}}\left(\sigma_{n-1} \ldots \sigma_{1} ; x\right)=\prod_{\lambda=k I, k \in \mathrm{N}}\left(x^{|I|}-1\right)^{l_{I}}
$$

according to Proposition 6.17.

This gives the characteristic polynomial of any permutation $\sigma_{i-1} \sigma_{i-2} \ldots \sigma_{1}$ in the regular representation of $\mathfrak{S}_{n}$,

$$
P_{\mathfrak{S}_{n}}\left(\sigma_{i-1} \ldots \sigma_{1} ; x\right)=P_{\mathfrak{S}_{i}}\left(\sigma_{i-1} \ldots \sigma_{1} ; x\right)^{n ! / i !}
$$

Example 6.20 The characteristic polynomial of $\sigma_{n-1} \ldots \sigma_{1}$ in any irreducible representation of $\mathfrak{S}_{n}$ is a product of cyclotomic polynomials. Table 1 shows these polynomials for $n \leq 6$. 


\begin{tabular}{|c|c|}
\hline Representation & Vharacteristic polynomial \\
\hline 2 & $x-1$ \\
11 & $x+1$ \\
\hline 3 & $x-1$ \\
21 & $1+x+x^{2}$ \\
111 & $x-1$ \\
\hline 4 & $x-1$ \\
31 & $(x-1)\left(x^{2}+1\right)$ \\
22 & $x^{2}-1$ \\
211 & $x^{3}+x^{2}+x+1$ \\
1111 & $x+1$ \\
\hline 5 & $x-1$ \\
41 & $x^{4}+x^{3}+x^{2}+x+1$ \\
32 & $x^{5}-1$ \\
311 & $\left(x^{5}-1\right)(x-1)$ \\
221 & $x^{5}-1$ \\
2111 & $x^{4}+x^{3}+x^{2}+x+1$ \\
11111 & $x-1$ \\
\hline 6 & $x-1$ \\
51 & $x^{5}-x^{4}+x^{3}-x^{2}+x-1$ \\
42 & $\left(x^{6}-1\right)\left(x^{3}+1\right)$ \\
411 & $(x+1)\left(x^{6}-1\right)\left(x^{3}-1\right)$ \\
33 & $\left(x^{3}-1\right)\left(x^{2}-1\right)$ \\
321 & $\left(x^{4}+x^{2}+1\right)^{3}\left(x^{2}-1\right)^{2}$ \\
3111 & $(x-1)\left(x^{6}-1\right)\left(x^{3}+1\right)$ \\
222 & $\left(x^{3}+1\right)\left(x^{2}-1\right)$ \\
2211 & $\left(x^{6}-1\right)\left(x^{3}-1\right)$ \\
21111 & $x^{5}+x^{4}+x^{3}+x^{2}+x+1$ \\
111111 & $x+1$ \\
\hline
\end{tabular}

\subsection{Diagonal Hyperplane Arrangements}

\subsubsection{The Quantum Bilinear form $B_{n}$}

Varchenko associated to every real configuration $\mathcal{C}$ of hyperplanes ${ }^{\S}$ a bilinear form $B_{\mathcal{C}}$, called the quantum bilinear form of $\mathcal{C}$, which is defined as follows. One first associates to each hyperplane $H$ of $\mathcal{C}$ a weight $a_{H}$ in some fixed commutative ring. An edge of $\mathcal{C}$ is any nonempty intersection of some subset of the hyperplanes of $\mathcal{C}$. The weight $a_{E}$ of an edge $E$ is defined as the product of the weights of all the hyperplanes containing $E$. The set of all edges of $\mathcal{C}$ is denoted by $\mathcal{E}_{\mathcal{C}}$.

The connected components of the complement of the hyperplanes of $\mathcal{C}$ are called domains. Denote by $\mathcal{D}_{\mathcal{C}}$ the set of all domains of $\mathcal{C}$. Let also $A_{\mathcal{C}}$ be the ring of polynomials in the commutative variables $\left(a_{H}\right)_{H \in \mathcal{C}}$. The quantum bilinear form $B_{\mathcal{C}}$ associated with $\mathcal{C}$ is the bilinear form on the space $M_{\mathcal{C}}$ of the $A_{\mathcal{C}}$-linear combinations of the domains of $\mathcal{D}_{\mathcal{C}}$ which is defined by

$$
B_{\mathcal{C}}(P, Q)=\prod a_{H}
$$

the product being taken over all hyperplanes $H \in \mathcal{C}$ separating the domains $P$ and $Q$. Varchenko showed that the determinant of the form $B_{\mathcal{C}}$ is given by the following formula

$$
\operatorname{det} B_{\mathcal{C}}=\prod_{E \in \mathcal{E}_{C}}\left(1-a_{E}^{2}\right)^{m(E)}
$$

\footnotetext{
$\S$ A configuration of hyperplanes is any finite set of hyperplanes in some affine or projective space.
} 
where $m(E)$ is an integer, called the multiplicity of the edge $E$ (see Sect. 2 of [13] for more details).

Consider now the configuration $\mathcal{D}_{n}$ of diagonal hyperplanes: it consists of the hyperplanes $H_{i j}$ of $\mathbf{R}^{n}$ defined by $x_{i}=x_{j}$ for $1 \leq i<j \leq n$. Let $a_{j i}$ be the weight of the hyperplane $H_{i j}$. Here, the domains are the cones $P_{\sigma}$ defined by

$$
P_{\sigma}=\left\{\left(x_{1}, \ldots, x_{n}\right) \in \mathbf{R}^{n}, x_{\sigma(1)}<x_{\sigma(2)}<\cdots<x_{\sigma(n)}\right\}
$$

for all permutations $\sigma$ of $\mathfrak{S}_{n}$. The quantum bilinear form $B_{\mathcal{D}_{n}}=B_{n}$ associated with $\mathcal{D}_{n}$ can therefore be considered as a bilinear form on $\mathbf{Z}\left[a_{j i}\right]\left[\mathfrak{S}_{n}\right]$. The matrix of $B_{n}$ is given by

$$
B_{n}(\sigma, \tau)=\prod_{\substack{\left(\sigma^{-1}(i)-\sigma^{-1}(j)\right)\left(\tau^{-1}(i)-\tau^{-1}(j)\right)<0 \\ 1 \leq i<j \leq n}} a_{j i}
$$

for $\sigma, \tau \in \mathfrak{S}_{n}$. In other words, the entry of order $(\sigma, \tau)$ of $B_{n}$ is obtained by taking the products of all $a_{j i}$ for which the pair $j i$ does not appear in the same order in $\tau$ and $\sigma$, i.e. for which $\sigma=\ldots i \ldots j \ldots$ and $\tau=\ldots j \ldots i \ldots$ (or the converse). The bilinear form $B_{n}$ can also be interpreted as the contravariant form of a suitable quantum group (cf. Schechtman [36]).

Varchenko's formula (41) reduces here to

$$
\operatorname{det} B_{n}=\prod_{I_{k}}\left(1-\prod_{(j, i) \in I_{k}} a_{j i}^{2}\right)^{n_{k}}
$$

where $I_{k}$ runs through all subsets of $k(k-1) / 2$ elements of $\{(j, i), 1 \leq i<j \leq n\}$ for $k \in[2, n]$ and where $n_{k}$ denotes the integer $(k-2) !(n-k+1) !$.

Finally, relation (42) shows that $B_{n}$ is the matrix of an element of $\mathbf{Z}\left[a_{j i}\right]\left[\mathfrak{S}_{n}\right]$ (considered as an operator in the regular representation of $\mathfrak{S}_{n}$ ) iff $a_{j i}=a_{k l}$ for all $j>i$ and $k>l$. If we call $q$ this common value, we see that $B_{n}=U_{n}(q)$. Thus, $U_{n}(q)$ has the same matrix as the quantum bilinear form associated with the diagonal hyperplane arrangement when every hyperplane as the same weight $q$ and one sees that Corollary 4.13 can be also obtained by specializing formula (43).

Example 6.21 The matrix of the quantum bilinear form $B_{3}$ is

$\begin{array}{ccccccc}123 & 132 & 213 & 231 & 312 & 321 \\ 123 \\ 132 \\ 213 \\ 231 \\ 312 \\ 321\end{array}\left(\begin{array}{cccccc}1 & a_{32} & a_{21} & a_{21} a_{31} & a_{31} a_{32} & a_{21} a_{31} a_{32} \\ a_{32} & 1 & a_{21} a_{32} & a_{21} a_{31} a_{32} & a_{31} & a_{21} a_{31} \\ a_{21} & a_{21} a_{32} & 1 & a_{31} & a_{21} a_{31} a_{32} & a_{31} a_{32} \\ a_{21} a_{31} & a_{21} a_{31} a_{32} & a_{31} & 1 & a_{21} a_{32} & a_{32} \\ a_{31} a_{32} & a_{31} & a_{21} a_{31} a_{32} & a_{21} a_{32} & 1 & a_{21} \\ a_{21} a_{31} a_{32} & a_{21} a_{31} & a_{31} a_{32} & a_{32} & a_{21} & 1\end{array}\right)$

In this case,

$$
\operatorname{det} B_{3}=\left(1-a_{21}^{2}\right)^{2}\left(1-a_{31}^{2}\right)^{2}\left(1-a_{32}^{2}\right)^{2}\left(1-\left(a_{21} a_{31} a_{32}\right)^{2}\right)
$$

$B_{3}$ is the matrix of an element of $\mathbf{Z}\left[a_{21}, a_{31}, a_{32}\right]\left[\mathfrak{S}_{3}\right]$ iff $a_{21}=a_{31}=a_{32}$. 


\subsubsection{A decomposition of $B_{n}$}

We shall now see that one can recover formula (43) from a factorization of $B_{n}$ which is in fact a specialization of Corollary 6.14. We shall therefore define first a representation $\mu_{n}$ of the braid group $\mathcal{B}_{n}$. For $n \geq 2$ and $i \in[1, n-1]$, consider the $\mathfrak{S}_{n} \times \mathfrak{S}_{n}$ matrix $S_{i}^{(n)}$ defined by

$$
S_{i}^{(n)}(\sigma, \tau)=\left\{\begin{array}{cl}
\tilde{a}_{\sigma(i) \sigma(i+1)} & \text { if } \tau=\sigma \sigma_{i} \\
0 & \text { in all other cases }
\end{array}\right.
$$

where $\widetilde{a}_{i j}$ denotes $a_{j i}$ if $j>i$ and $a_{i j}$ if $j<i$. These matrices are obtained from the diagonal solutions $R\left(e_{i} \otimes e_{j}\right)=\widetilde{a}_{i j} e_{i} \otimes e_{j}$ of the Yang-Baxter equation by restriction of $\check{R}$ to the $n$ !-dimensional subspace of $V^{\otimes n}$ spanned by multilinear elements $e_{\sigma(1)} \otimes \cdots \otimes e_{\sigma(n)}, \sigma \in \mathfrak{S}_{n}$. Thus,

$$
\left\{\begin{array}{clrl}
S_{i}^{(n)} S_{j}^{(n)} & =S_{j}^{(n)} S_{i}^{(n)} & & \text { if }|i-j|>1 \\
S_{i}^{(n)} S_{i+1}^{(n)} S_{i}^{(n)} & =S_{i+1}^{(n)} S_{i}^{(n)} S_{i+1}^{(n)} & & \text { if } i \in[1, n-2]
\end{array}\right.
$$

so that

$$
\mu_{n}\left(s_{i}\right)=S_{i}^{(n)}
$$

defines a representation of $\mathcal{B}_{n}$. The matrix of Varchenko's quantum bilinear form is then

$$
B_{n}=\mu_{n}\left(\mathcal{U}_{n}\right)=\sum_{\sigma \in \mathcal{S}_{n}} \mu_{n}\left(T_{\sigma}\right)
$$

the results of Sect. 6.3 can be applied. The images $T_{k}^{(n)}=\mu_{n}\left(\mathcal{T}_{k}\right)$ are the $\mathfrak{S}_{n} \times \mathfrak{S}_{n}$ matrices

$$
T_{k}^{(n)}=I+S_{k-1}^{(n)}+S_{k-1}^{(n)} S_{k-2}^{(n)}+\cdots+S_{k-1}^{(n)} S_{k-2}^{(n)} \ldots S_{1}^{(n)}
$$

whose elements are

$$
T_{k}^{(n)}(\sigma, \tau)= \begin{cases}1 & \text { if } \sigma=\tau \\ \prod_{i=l}^{k-1} \widetilde{a}_{\sigma(k) \sigma(l)} & \text { if } \tau=\sigma \sigma_{k-1} \ldots \sigma_{l} \quad \text { with } l=1, k-1 \\ 0 & \text { in all other cases }\end{cases}
$$

In other words, $T_{k}^{(n)}$ is the matrix obtained from $B_{n}$ by taking all entries indexed by a pair of the form $\left(\sigma, \sigma \sigma_{k-1} \ldots \sigma_{l}\right)$ with some $l \in[1, n]$ and by replacing all other entries by 0 . The first formula of Corollary 6.14 reduces then to the following:

\section{Proposition 6.22}

$$
B_{n}=T_{2}^{(n)} \ldots T_{n}^{(n)}
$$


Example 6.23 For $n=3$, one has $B_{3}=T_{2}^{(3)} T_{3}^{(3)}$, with

$$
\begin{aligned}
& \begin{array}{llllll}
123 & 132 & 213 & 231 & 312 & 321
\end{array} \\
& T_{2}^{(3)}=\begin{array}{l}
123 \\
132 \\
213 \\
231 \\
312 \\
321
\end{array}\left(\begin{array}{cccccc}
1 & 0 & a_{21} & 0 & 0 & 0 \\
0 & 1 & 0 & 0 & a_{31} & 0 \\
a_{21} & 0 & 1 & 0 & 0 & 0 \\
0 & 0 & 0 & 1 & 0 & a_{32} \\
0 & a_{31} & 0 & 0 & 1 & 0 \\
0 & 0 & 0 & a_{32} & 0 & 1
\end{array}\right) \\
& \begin{array}{llllll}
123 & 132 & 213 & 231 & 312 & 321
\end{array} \\
& T_{3}^{(3)}=\begin{array}{l}
123 \\
132 \\
213 \\
231 \\
312 \\
321
\end{array}\left(\begin{array}{cccccc}
1 & a_{32} & 0 & 0 & a_{31} a_{32} & 0 \\
a_{32} & 1 & a_{21} a_{32} & 0 & 0 & 0 \\
0 & 0 & 1 & a_{31} & 0 & a_{31} a_{32} \\
a_{21} a_{31} & 0 & a_{31} & 1 & 0 & 0 \\
0 & 0 & 0 & a_{21} a_{32} & 1 & a_{21} \\
0 & a_{21} a_{31} & 0 & 0 & a_{21} & 1
\end{array}\right)
\end{aligned}
$$

The second formula of Corollary 6.14 applied to Varchenko's quantum bilinear form will give us a factorization of the matrix $T_{k}^{(n)}$ which reduces to the decomposition (23) when all the $a_{j i}$ are specialized to $q$. As in the $T_{n}(q)$ case, it will give us a closed formula for the inverse of $T_{k}^{(n)}$ (and hence of $B_{n}$ ) and for its determinant.

For $n \geq 2, k \in[2, n], i \in[2, k]$ and $j \in[1, k-1]$, we define $\mathfrak{S}_{n} \times \mathfrak{S}_{n}$-matrices $\Gamma_{\gamma_{i, k}}^{(n, k)}$ and $\bar{\Gamma}_{\gamma_{j, k}^{\prime}}^{(n, k)}$ by

$$
\begin{array}{r}
\Gamma_{\gamma_{i, k}}^{(n, k)}(\sigma, \tau)=\left\{\begin{array}{cc}
\widetilde{a}_{\sigma(k-1) \sigma(k)}^{2} \prod_{l=k-i+1}^{k-2} \widetilde{a}_{\sigma(k-1) \sigma(l)} & \text { if } \tau=\sigma \gamma_{i, k} \\
0 & \text { in all other cases }
\end{array}\right. \\
\bar{\Gamma}_{\gamma_{j, k}^{\prime}}^{(n, k)}(\sigma, \tau)=\left\{\begin{array}{cc}
\prod_{l=k-j}^{k-1} \widetilde{a}_{\sigma(k) \sigma(l)} & \text { if } \tau=\sigma \gamma_{j, k}^{\prime} \\
0 & \text { in all other cases }
\end{array}\right.
\end{array}
$$

where

$$
\begin{aligned}
\gamma_{i, k} & =(1 \ldots k-i k-1 k-i+1 \ldots k-2 k \ldots n) \\
\gamma_{j, k}^{\prime} & =(1 \ldots k-j-1 k k-j \ldots k-1 k+1 \ldots n)
\end{aligned}
$$

The specialization of the second identity of Corollary 6.14 gives here the following factorization.

Proposition 6.24 For $n \geq 2$ and $k \in[2, n]$,

$$
T_{k}^{(n)}=\left(I-\Gamma_{\gamma_{k, k}}^{(n, k)}\right) \ldots\left(I-\Gamma_{\gamma_{2, k}}^{(n, k)}\right)\left(I-\bar{\Gamma}_{\gamma_{1, k}^{\prime}}^{(n, k)}\right)^{-1} \ldots\left(I-\bar{\Gamma}_{\gamma_{k-1, k}^{\prime}}^{(n, k)}\right)^{-1} .
$$


Example 6.25 For $k=3$ and $n=3$, the different matrices involved in (48) are given below:

$$
\begin{aligned}
& \begin{array}{llllll}
123 & 132 & 213 & 231 & 312 & 321
\end{array} \\
& I-\Gamma_{(213)}^{(3,3)}=\left(\begin{array}{cccccc}
1 & 0 & -a_{32}^{2} a_{21} & 0 & 0 & 0 \\
0 & 1 & 0 & 0 & -a_{32}^{2} a_{31} & 0 \\
-a_{31}^{2} a_{21} & 0 & 1 & 0 & 0 & 0 \\
0 & 0 & 0 & 1 & 0 & -a_{31}^{2} a_{32} \\
0 & -a_{21}^{2} a_{31} & 0 & 0 & 1 & 0 \\
0 & 0 & 0 & -a_{21}^{2} a_{32} & 0 & 1
\end{array}\right) \\
& \begin{array}{llllll}
123 & 132 & 213 & 231 & 312 & 321
\end{array} \\
& I-\Gamma_{(123)}^{(3,3)}=\left(\begin{array}{cccccc}
1-a_{32}^{2} & 0 & 0 & 0 & 0 & 0 \\
0 & 1-a_{32}^{2} & 0 & 0 & 0 & 0 \\
0 & 0 & 1-a_{31}^{2} & 0 & 0 & 0 \\
0 & 0 & 0 & 1-a_{31}^{2} & 0 & 0 \\
0 & 0 & 0 & 0 & 1-a_{21}^{2} & 0 \\
0 & 0 & 0 & 0 & 0 & 1-a_{21}^{2}
\end{array}\right) \\
& \begin{array}{llllll}
123 & 132 & 213 & 231 & 312 & 321
\end{array} \\
& I-\bar{\Gamma}_{(132)}^{(3,3)}=\left(\begin{array}{cccccc}
1 & -a_{32} & 0 & 0 & 0 & 0 \\
-a_{32} & 1 & 0 & 0 & 0 & 0 \\
0 & 0 & 1 & -a_{31} & 0 & 0 \\
0 & 0 & -a_{31} & 1 & 0 & 0 \\
0 & 0 & 0 & 0 & 1 & -a_{21} \\
0 & 0 & 0 & 0 & -a_{21} & 1
\end{array}\right) \\
& I-\bar{\Gamma}_{(312)}^{(3,3)}=\left(\begin{array}{cccccc}
123 & 132 & 213 & 231 & 312 & 321 \\
1 & 0 & 0 & 0 & -a_{31} a_{32} & 0 \\
0 & 1 & -a_{21} a_{32} & 0 & 0 & 0 \\
0 & 0 & 1 & 0 & 0 & -a_{31} a_{32} \\
-a_{21} a_{31} & 0 & 0 & 1 & 0 & 0 \\
0 & 0 & 0 & -a_{21} a_{32} & 1 & 0 \\
0 & -a_{21} a_{31} & 0 & 0 & 0 & 1
\end{array}\right)
\end{aligned}
$$

In this case, (48) reduces to

$$
T_{3}^{(3)}=\left(I-\Gamma_{(213)}^{(3,3)}\right)\left(I-\Gamma_{(123)}^{(3,3)}\right)\left(I-\bar{\Gamma}_{(132)}^{(3,3)}\right)^{-1}\left(I-\bar{\Gamma}_{(312)}^{(3,3)}\right)^{-1}
$$


and

$$
T_{2}^{(3)}=\left(I-\Gamma_{(123)}^{(3,2)}\right)\left(I-\bar{\Gamma}_{(213)}^{(3,2)}\right)^{-1}
$$

Note 6.26 One can check that

$$
\left(I-\Gamma_{\gamma_{i, k}}^{(n, k)}\right)\left(\sum_{l=0}^{i-2}\left(\Gamma_{\gamma_{i, k}}^{(n, k)}\right)^{l}\right)=\Delta_{i}^{(n, k)}
$$

where $\Delta_{i}^{(n, k)}$ is the diagonal matrix

$$
\Delta_{i}^{(n, k)}(\sigma, \tau)=\left\{\begin{array}{cl}
1-\prod_{k-i+1 \leq r<s \leq k} \widetilde{a}_{\sigma(r) \sigma(s)}^{2} & \text { if } \sigma=\tau \\
0 & \text { if } \sigma \neq \tau
\end{array}\right.
$$

One has $\Delta_{n}^{(n, n)}=\left(1-\prod_{1 \leq i<j \leq n} a_{j i}^{2}\right) I$ and

$$
\left(I-\bar{\Gamma}_{\gamma_{j, k}^{\prime}}^{(n, k)}\right)\left(\sum_{l=0}^{j}\left(\bar{\Gamma}_{\gamma_{j, k}^{\prime}}^{(n, k)}\right)^{l}\right)=\Delta_{j+1}^{(n, k)}
$$

Formulas (49) and (50) both give a more explicit version of decomposition (48) and a closed form for the inverse of $T_{k}^{(n)}$ (and hence also for the inverse of $B_{n}$ ).

Note 6.27 It can also be checked that

$$
\operatorname{det}\left(I-\Gamma_{\gamma_{i, k}}^{(n, k)}\right)=\left(\prod_{\substack{I \subset[1, n] \\|I|=i}}\left(1-\prod_{\substack{r, s \in I \\ r<s}} a_{s r}^{2}\right)\right)^{m_{n, i}}
$$

where

$$
m_{n, i}=\frac{i n !}{2\left(\begin{array}{c}
i \\
2
\end{array}\right)\left(\begin{array}{l}
n \\
i
\end{array}\right)}=\frac{(n-i) ! i !}{i-1}
$$

On the other hand,

$$
\operatorname{det}\left(I-\bar{\Gamma}_{\gamma_{j, k}^{\prime}}^{(n, k)}\right)=\left(\prod_{\substack{I \subset[1, n] \\|I|=j+1}}\left(1-\prod_{\substack{r, s \in I \\ r<s}} a_{r s}^{2}\right)\right)^{m_{n, j}^{\prime}}
$$

with

$$
m_{n, j}^{\prime}=\frac{j n !}{2\left(\begin{array}{c}
j+1 \\
2
\end{array}\right)\left(\begin{array}{c}
n \\
j+1
\end{array}\right)}=(n-j-1) ! j ! .
$$

It is interesting to observe that (51) and (52) show that the determinants of $I-\Gamma_{\gamma_{i, k}}^{(n, k)}$ and $I-\bar{\Gamma}_{\gamma_{j, k}^{\prime}}^{(n, k)}$ are independent of $k$. 


\subsection{The $t$-shuffle Operator in the Hecke Algebra}

The representations of the braid group considered in the foregoing sections were all obtained from trivial (diagonal) solutions of the Yang-Baxter equation. The simplest non-trivial solutions are those which factorize through the Hecke algebra $H_{n}(q)$. It is therefore of interest to consider the element

$$
V_{n}(t)=\sum_{\sigma \in \mathfrak{S}_{n}} t^{\ell(\sigma)} T_{\sigma}
$$

of $H_{n}(q) \otimes \mathbf{C}[t]$, i.e. the image of $\mathcal{U}_{n}$ under the morphism from the braid group $\mathcal{B}_{n}$ into $H_{n}(q)$ defined by $s_{i} \rightarrow t T_{i}$. All the factorizations of Sect. 6.3.2 are valid for $V_{n}(t)$, and we shall also obtain an explicit formula for the determinant of this operator, which can be regarded as a $q$-analogue of the determinant formula for $U_{n}(t)$.

According to the results of Sect. 6.3.2, it is sufficient to compute the determinants of the elements

$$
1-T_{n-1} \ldots T_{1} \quad \text { and } \quad 1-T_{n-1}^{2} \ldots T_{1}
$$

considered considered as operators of the (left or right) regular representation of $H_{n}(q)$. But one has

$$
\begin{aligned}
& \operatorname{det}\left(1-T_{n-1} \ldots T_{1}\right)=\left.\left(\prod_{\lambda \vdash n} P_{V_{\lambda}}\left(T_{n-1} \ldots T_{1} ; x\right)^{f_{\lambda}}\right)\right|_{x=1} \\
& \operatorname{det}\left(1-T_{n-1}^{2} \ldots T_{1}\right)=\left.\left(\prod_{\lambda \vdash n} P_{V_{\lambda}}\left(T_{n-1}^{2} \ldots T_{1} ; x\right)^{f_{\lambda}}\right)\right|_{x=1}
\end{aligned}
$$

where $f_{\lambda}$ is the number of standard Young tableaux of shape $\lambda$ and where $P_{V_{\lambda}}(s ; x)$ is the characteristic polynomial det $(1-x s)$ of $s \in H_{n}(q)$ in the irreducible representation of $H_{n}(q)$ indexed by $\lambda$ (see, for example: [31]).

Let $K_{\lambda, \mu}=\left\langle s_{\lambda}, h_{\mu}\right\rangle$ be the Kostka numbers. The following proposition expresses the characteristic polynomial of $T_{n-1} \cdots T_{1}$ in the $q$-Specht module $V_{\lambda}(q)$ in terms of the corresponding polynomial for the symmetric group, as computed in Sect. 6.3.3.

Proposition 6.28 Let $\lambda$ be a partition of $n$ and $V_{\lambda}(q)$ be the corresponding irreducible representation of $H_{n}(q)$. Then, the characteristic polynomial of $T_{n-1} T_{n-2} \ldots T_{1}$ in $V_{\lambda}(q)$ is

$$
P_{V_{\lambda}(q)}\left(T_{n-1} T_{n-2} \ldots T_{1} ; x\right)=P_{V_{\lambda}(1)}\left(\sigma_{n-1} \sigma_{n-2} \ldots \sigma_{1} ; q^{(n-1) \alpha_{\lambda}} x\right)
$$

where $V_{\lambda}(1)$ is the Specht module for the symmetric group, and $\alpha_{\lambda}=K_{\lambda, 21^{n-2}} / K_{\lambda, 1^{n}}$.

Proof The existence of a homogeneity factor between the characteristic polynomials of $T_{n-1} \ldots T_{1}$ and $\sigma_{n-1} \ldots \sigma_{1}$ can be seen on the Kazhdan-Lusztig model of the irreducible $H_{n}(q)$-module $V_{\lambda}$, and this factor is determined by the equality

$$
\operatorname{det}_{V_{\lambda}}\left(T_{n-1} \ldots T_{1}\right)=\left(\operatorname{det}_{V_{\lambda}} T_{1}\right)^{n-1}=q^{(n-1) K_{\lambda, 21^{n-2}}}
$$

which is easily checked. 
Corollary 6.29 The characteristic polynomial of $T_{n-1}^{2} T_{n-2} \ldots T_{1}$ in the irreducible $H_{n}(q)$-module $V_{\lambda}(q)$ is equal up to a homogeneity factor to the characteristic polynomial of the corresponding permutation in the irreducible $\boldsymbol{S}_{n}$-module $V_{\lambda}(1)$, i.e.

$$
P_{V_{\lambda}}\left(T_{n-1}^{2} T_{n-2} \ldots T_{1} ; x\right)=P_{W_{\lambda}}\left(\sigma_{n-2} \ldots \sigma_{1} ; q^{n \alpha_{\lambda}} x\right)
$$

where $\alpha_{\lambda}=K_{\lambda, 21^{n-2}} / K_{\lambda, 1^{n}}$.

Example 6.30 For $|\lambda| \leq 4$, these polynomials are

\begin{tabular}{|c|c|c|}
\hline$\lambda$ & $P_{V_{\lambda}}\left(T_{n-1} \ldots T_{1} ; x\right)$ & $P_{V_{\lambda}}\left(T_{n-1}^{2} T_{n-2} \ldots T_{1} ; x\right)$ \\
\hline 2 & $1-x q$ & $1-x q^{2}$ \\
11 & $1+x$ & $1-x$ \\
\hline 3 & $1-x q^{2}$ & $1-x q^{3}$ \\
21 & $1+x q+x^{2} q^{2}$ & $1-q^{3} x^{2}$ \\
111 & $1-x$ & $1+x$ \\
\hline 4 & $1-x q^{3}$ & $1-q^{4} x$ \\
31 & $1-x q+x^{2} q^{2}-x^{3} q^{3}$ & $1-x^{3} q^{4}$ \\
22 & $1-x^{2} q^{3}$ & $1+x q^{2}+x^{2} q^{4}$ \\
2111 & $1+x q^{2}+x^{2} q^{4}+x^{3} q^{6}$ & $1-q^{8} x^{3}$ \\
1111 & $1+x$ & $1-x$ \\
\hline
\end{tabular}

Note 6.31 Consider the elements $\diamond_{i}(t)$ of $H_{n}(q)$ defined by

$$
\diamond_{i}(t)=1+t T_{i}
$$

for $i \in[1, n-1]$. These elements interpolate between two interesting elements of $H_{n}(q)$

$$
\diamond_{i}(1)=\square_{i}=1+T_{i}, \quad \diamond_{i}(-1 / q)=\frac{-1}{q} \nabla_{i}=\frac{-1}{q}\left(T_{i}-q\right)
$$

(see [31] for more details). The operators $\diamond_{i}(t)$ satisfy Yang-Baxter type relations

$$
\diamond_{i}(t)\left(\diamond_{i+1}(t)-\frac{1+t(q-1)}{2+t(q-1)}\right) \diamond_{i}(t)=\diamond_{i+1}(t)\left(\diamond_{i}(t)-\frac{1+t(q-1)}{2+t(q-1)}\right) \diamond_{i+1}(t)
$$

and as a special case of a construction of Cherednik, one can associate with the maximal permutation $\omega_{n}$ of $\mathfrak{S}_{n}$ the element

$$
\diamond_{\omega_{n}}=\diamond_{1}\left(\left(\diamond_{2}-f_{13}\right) \diamond_{1}\right) \ldots\left(\left(\diamond_{n-1}-f_{1 n}\right) \ldots\left(\diamond_{2}-f_{13}\right) \diamond_{1}\right)
$$

of $H_{n}(q) \otimes \mathbf{C}(t)$, where

$$
f_{1 k}=[1]_{t, q} \frac{[1]_{t, q}^{n-1}-1}{[1]_{t, q}^{n}-1}
$$

with $[1]_{t, q}=1+t(q-1)$. It would be interesting to clarify the relation between $\diamond_{\omega_{n}}$ and $V_{n}(t)$. These elements are clearly related in the two extreme cases $t=1$ and $t=-1 / q$ but we do not know if such relations still exist in the general case. 


\section{Deformations of the Algebra of Noncommutative Symmetric Func- tions}

\subsection{Deformations and Lie Idempotents}

This section is devoted to a special case of the deformations of the usual Cauchy and convolution products of $K\langle A\rangle$ obtained by means of noncommutative symmetric functions (cf. Sect. 3.2). We are here interested in the case where the set of primitive elements of the deformed algebra is still the free Lie algebra $L(A)$. This case is of some importance, since it leads to a complete understanding of a class of interesting decompositions of the free associative algebra.

We use the notations of Sect. 3.2. We will restrict the families $F=\left(F_{n}\right)_{n \geq 1}$ used to construct the deformations to those satifying for all $n$

$$
F_{n} * \Psi_{n}=k_{F_{n}} \Psi_{n}
$$

where $k_{F_{n}}$ is a constant. These equations have of course many solutions. An interesting class is given by $F_{n}=S_{n}(X A)$, where $X$ is a totally ordered alphabet (which can be virtual, i.e. defined by the specialization of a transcendance basis of $Q S y m)$. Indeed, the following result shows that such a family satisfies to the desired property:

Proposition 7.1 Let $X$ be a totally ordered commutative alphabet, let $A$ be a noncommutative alphabet and let $\pi_{n}$ be a homogenous element of weight $n$ in $L(\Psi)$. Then,

$$
S_{n}(X A) * \pi_{n}(A)=\psi_{n}(X) \pi_{n}(A)
$$

for all $n \geq 1$, where $\psi_{n}(X)$ is the usual power sum symmetric function.

Proof Using Proposition 2.1, we can write

$$
\begin{aligned}
\sigma(X A ; t) * \pi_{n}(A) & =\mu_{\infty}\left(\left(\bigotimes_{x \in X} \sigma(A ; x t)\right) * \Delta^{\infty}\left(\pi_{n}\right)\right) \\
& =\mu_{\infty}\left(\left(\bigotimes_{x \in X} \sigma(A ; x t)\right) * \sum 1 \otimes \cdots \otimes 1 \otimes \pi_{n} \otimes 1 \ldots\right)
\end{aligned}
$$

from which it follows that

$$
\sigma(X A ; t) * \pi_{n}(A)=\sum_{x \in X}\left(S_{n} * \pi_{n}\right) x^{n} t^{n}=\left(\sum_{x \in X} x^{n}\right) \pi_{n} t^{n}=\psi_{n}(X) \pi_{n} t^{n}
$$

Let now $X$ denote a fixed totally ordered commutative alphabet. According to Sect. 3, one can deform the ordinary Cauchy product of $K\langle A\rangle$ by means of the family $\left(\alpha\left(S_{n}(X A)\right)_{n \geq 1}\right.$. The product $\odot_{X}$ obtained by this method is here given by

$$
u \odot_{X} v=\left(\left(u \cdot \alpha\left(S_{n}(X A)\right)^{*-1}\right)\left(v \cdot \alpha\left(S_{m}(X A)\right)^{*-1}\right)\right) \cdot \alpha\left(S_{n+m}(X A)\right)
$$


for words $u$ and $v$ of respective lengths $n$ and $m$. The associated convolution algebra is still equal here to Solomon's descent algebra. It can be interpreted as the $K$-vector space Sym of noncommutative symmetric functions endowed with the new product $\cdot X$ defined by

$$
U \cdot{ }_{X} V=S_{n+m}(X A) *(U V)
$$

for $U \in \mathbf{S y m}_{n}$ and $V \in \mathbf{S y m}_{m}$ (cf. Sect. 3.2). The deformed interpretation morphism $\alpha_{X}$ is here

$$
\alpha_{X}(U)=S_{n}(X A) * U * S_{n}\left(\frac{1}{X} A\right)=S_{n}(X A) * U\left(\frac{1}{X} A\right)
$$

and in particular the interpretation of the Eulerian Lie idempotent $\Phi_{n}$ is

$$
\alpha_{X}\left(\Phi_{n}\right)=S_{n}(X A) * \Phi_{n}\left(\frac{1}{X} A\right)=\psi_{n}(X) \Phi_{n}\left(\frac{1}{X} A\right)=\frac{\Phi_{n}\left(\frac{1}{X} A\right)}{\psi_{n}\left(\frac{1}{X}\right)}
$$

according to Proposition 7.1. This situation is interesting since the forthcoming proposition shows that every Lie idempotent of Solomon's descent algebra can be described in this way.

Proposition 7.2 Let A be a noncommutative alphabet and let $\pi_{n}$ be an arbitrary homogeneous Lie idempotent of order $n$ in $L(\Psi)$. The following properties are equivalent:

1. $\Pi_{n}$ is a homogenous Lie idempotent of order $n$ in $L(\Psi)$.

2. There exists a virtual totally ordered commutative alphabet $X$ such that

$$
\Pi_{n}=\frac{\pi_{n}(X A)}{\psi_{n}(X)}
$$

where $\psi_{n}$ is the usual power sum symmetric function.

Proof $(2 \Longrightarrow 1$ ) follows essentially from Proposition 7.1. Indeed, this allows us to write

$$
\Pi_{n} * \pi_{n}=\frac{1}{\psi_{n}(X)} \pi_{n}(X A) * \pi_{n}=\frac{1}{\psi_{n}(X)} \pi_{n} * S_{n}(X A) * \pi_{n}=\pi_{n} * \pi_{n}=\pi_{n}
$$

On the other hand,

$$
\pi_{n} * \Pi_{n}=\frac{1}{\psi_{n}(X)} \pi_{n} * \pi_{n}(X A)=\frac{1}{\psi_{n}(X)} \pi_{n} * \pi_{n} * S_{n}(X A)=\frac{1}{\psi_{n}(X)} \pi_{n} * S_{n}(X A)=\Pi_{n}
$$

so that $\pi_{n} * \Pi_{n}=\Pi_{n}$ and $\Pi_{n} * \pi_{n}=\pi_{n}$ for all $n \geq 1$, which implies that $\Pi_{n}$ is a Lie idempotent of $L(\Psi)$.

$(1 \Longrightarrow 2)$ Consider a totally ordered commutative alphabet $X$. Let $\left(\left[\pi^{L}\right]\right)_{L \in L y}$ be the Lyndon basis of the free Lie algebra $L(\Psi)$ associated with the generating family $\left(\pi_{n}\right)_{n \geq 1}$ (where $L y$ denotes the set of Lyndon words over the alphabet $\mathbf{N}$ ). From the case $\pi_{n}=\Psi_{n}$, one can see that there exists a family $\left(p_{L}(X)^{(n)}\right)_{L \in L y_{n}}$ of quasi-symetric functions such that

$$
\pi_{n}(X A)=\sum_{L \in L y_{n}} p_{L}(X)\left[\pi^{L}\right]
$$


where $L y_{n}$ denotes the set of Lyndon words over $\mathbf{N}$ of weight $n$. The coefficient $p_{(n)}(X)$ of $\pi_{n}$ in this expansion is equal to $\psi_{n}(X)$, since

$$
\left.p_{(n)}(X) \pi_{n}=\pi_{(} X A\right) * \pi_{n}=\pi_{n} * S_{n}(X A) * \pi_{n}=\psi_{n}(X) \pi_{n} * \pi_{n}=\psi_{n}(X) \pi_{n}
$$

according to Proposition 7.1.

We shall now prove that the family $\left(p_{L}(X)\right)_{L \in L y_{n}, n \geq 1}$ is a transcendance basis for the algebra QSym of quasi-symmetric functions over $X$ (which is a free commutative algebra according to a result of Malvenuto and Reutenauer [22]). Let $\left(\pi_{I}^{*}\right)$ be the dual basis of $\left(\pi^{I}\right)$. As shown in [22], the family $\left(\pi_{L}^{*}\right)_{L \in L y_{n}, n \geq 1}$ is a transcendance basis of $Q s y m$. On the other hand, using the Cauchy formula, one can write

$$
S_{n}(X A)=\sum_{I \vdash n} \pi_{I}^{*}(X) \pi^{I}
$$

Expanding $S_{n}(A)$ on the basis $\left(\pi^{I}\right)$ and applying (53), one obtains another expression of $S_{n}(X A)$ on the $\left(\pi^{I}\right)$ basis. Identifying the coefficients, one finds

$$
\pi_{L}^{*}=k_{L} p_{L}+\sum_{\substack{L_{1}, \ldots, L_{k} \\ k \geq 2}} p_{L_{1}} \ldots p_{L_{k}}
$$

for any Lyndon word $L$ of weight $n$, the sum being taken over Lyndon words $L_{1}, \ldots, L_{k}$ of length strictly less than $|L|$ (and whose total length is equal to $|L|$ ). Also, the constant $k_{L}$ must be non-zero, as follows from using a simple argument on the cardinality of finite transcendance bases. This shows that $\left(p_{L}\right)_{L \in L y_{n}, n>1}$ is a transcendance basis of $Q S y m$, as required.

Let now $\Pi_{n}$ be an arbitrary Lie idempotent in $L(\Psi)$. One can expand it on the Lyndon basis associated with the family $\left(\pi_{n}\right)$, i.e.

$$
\Pi_{n}=\sum_{L \in L y_{n}} q_{L}\left[\pi^{L}\right]
$$

where the $q_{L}$ are scalars. According to the previous discussion, one can now define a totally ordered commutative (virtual) alphabet $X$ by setting $p_{L}(X)=q_{L}$ for every $L \in L y$. Since $q_{(n)}$ is here necessarily equal to 1 , it follows that

$$
\frac{\pi_{n}(X A)}{\psi_{n}(X)}=\frac{\pi_{n}(X A)}{p_{(n)}(X)}=\frac{\pi_{n}(X A)}{q_{(n)}}=\pi_{n}(X A)=\Pi_{n}
$$

as required.

Recall now that the Lie (quasi) idempotent

$$
\alpha_{X}\left(\Phi_{n}\right)=\Phi_{n} \frac{\left(\frac{1}{X} A\right)}{\psi_{n}\left(\frac{1}{X}\right)}
$$

is the element of $\mathbf{S y m}_{n}$ corresponding to projection onto the free Lie algebra with respect to the decomposition

$$
K\langle A\rangle=K \oplus L(A) \oplus(L(A), L(A))_{\odot_{x}} \oplus \ldots \oplus(\underbrace{L(A), \ldots, L(A)}_{n \text { terms }})_{\odot x} \oplus \ldots
$$


where $(L(A), \ldots, L(A))_{\odot x}$ is the submodule of $K\langle A\rangle$ spanned by the $\odot_{X}$-symmetrized products

$$
\left(P_{1}, \ldots, P_{n}\right)_{\odot_{X}}=\frac{1}{n !} \sum_{\sigma \in S G_{n}} P_{\sigma(1)} \odot_{X} \cdots \odot_{X} P_{\sigma(n)}
$$

of Lie polynomials. Hence it follows from Proposition 7.2 that all Lie idempotents of the descent algebra are the Lie projectors associated to some deformation of the canonical decomposition of $K\langle A\rangle$ given by (54).

Note 7.3 Interesting specializations are obtained by taking $X=1-q$ and $X=1 /(1-q)$. In the latter case, the associated interpretation $\alpha_{q}$ is

$$
\alpha_{q}(F)=S_{n}\left(\frac{1}{1-q} A\right) * F((1-q) A)
$$

In particular,

$$
\alpha_{q}\left(\pi^{I}\right)=S_{n}\left(\frac{1}{1-q} A\right) * \pi_{n}((1-q) A)
$$

for every family $\left(\pi_{n}\right)_{n \geq 1}$ of homogeneous Lie idempotents. On the other hand [37],

$$
S_{n}\left(\frac{1}{1-q} A\right) * \pi^{I}=\sum_{\sigma \in \mathfrak{S}_{r}} \frac{q^{\operatorname{maj}_{I}(\sigma)}}{\left(1-q^{i_{\sigma(1)}}\right)\left(1-q^{i_{\sigma(1)}+i_{\sigma(2)}}\right) \ldots\left(1-q^{\left.i_{\sigma(1)}+\cdots+i_{\sigma(r)}\right)}\right.} \pi^{\sigma \cdot I}
$$

Hence,

$$
\alpha_{q}\left(\pi^{I}\right)=\sum_{\sigma \in \mathfrak{S}_{r}} \frac{q^{\operatorname{maj}_{I}(\sigma)}}{\left(1-q^{i_{\sigma(1)}}\right)\left(1-q^{i_{\sigma(1)}+i_{\sigma(2)}}\right) \ldots\left(1-q^{\left.i_{\sigma(1)}+\cdots+i_{\sigma(r)}\right)}\right.} \pi((1-q) A)^{\sigma \cdot I}
$$

But it has been shown [7] that the limit for $q \rightarrow 1$ of of $\pi_{n}((1-q) A) /\left(1-q^{n}\right)$ is always equal to $\Psi_{n} / n$. Thus,

$$
\lim _{q \rightarrow 1} \alpha_{q}\left(\pi^{I}\right)=\sum_{\sigma \in \mathfrak{S}_{r}} \frac{1}{i_{\sigma(1)}}\left(i_{\sigma(1)}+i_{\sigma(2)}\right) \ldots\left(i_{\sigma(1)}+\cdots+i_{\sigma(r)}\right) \Psi^{\sigma \cdot I}=E_{\lambda(I)}(\Psi)
$$

where $\lambda(I)$ denotes the partition obtained by sorting $I$. Hence the convolution algebra associated with the deformed Cauchy product corresponding to $S_{n}(A /(1-q))$ degenerates when $q \rightarrow 1$ into the commutative algebra generated by the elements $\left(E_{\lambda}(\Psi)\right)_{\lambda \vdash_{n}}$. One should observe that these elements form a family of orthogonal idempotents, similar to those of Garsia and Reutenauer ([1] - see alsc, Krob et al. [7]).

\subsection{Structure of Multihomogeneous Modules Associated with Lie Idempotents}

We first recall some results [7]. Let $\pi=\left(\pi_{n}\right)_{n \geq 1}$ a family of homogeneous Lie idempotents. One can decompose $S_{n}$ on the basis $\left(\pi^{I}\right)$, say

$$
S_{n}=\sum_{I} p_{I} \pi^{I}
$$


where $p_{I}$ are constants. One associates then with each partition $\lambda=\left(l_{1}, \ldots, l_{r}\right)$ of $n$ the noncommutative symmetric function $E_{\lambda}(\pi)$ defined by

$$
E_{\lambda}(\pi)=\sum_{\lambda(I)=\lambda} p_{I} \pi^{I}
$$

where $\lambda(I)$ denotes the partition obtained by reordering the components of $I$. As shown ir Krob et al. [7], these elements form a complete system of orthogonal idempotents. When $\pi$ is the family ( $\left.\Phi_{n}\right)$ of Eulerian idempotents, these idempotents reduce to the family described by Garsia and Reutenauer [1]. In this case, $E_{\lambda}(\Phi)$ is the projector on the module $\left(L(A)_{l_{1}}, \ldots, L(A)_{l_{r}}\right)$ in the decomposition

$$
K\langle A\rangle=K \oplus L(A) \oplus(L(A), L(A)) \oplus \ldots \oplus(\underbrace{L(A), \ldots, L(A)}_{n \text { terms }}) \oplus \ldots
$$

In the case of an arbitrary family of Lie idempotents, the structure of the target modules is not immediately clear. According to previous discussion, one can say that $E_{\lambda}(\pi)$ is the projector on $\left(L(A)_{l_{1}}, \ldots, L(A)_{l_{r}}\right)_{\odot_{X}}$ with respect to the decomposition (54) if one interprets the Lie idempotents $\pi_{n}$ as $\Phi_{n}(X A) / \psi_{n}(X)$ for some totally ordered alphabet $X$ (which is always possible according to Proposition 7.2). It remains, however, to give a more explicit description of the images of the $E_{\lambda}(\pi)$, i.e. of

$$
\operatorname{Im} E_{\lambda}(\pi)=K\langle A\rangle_{|\lambda|} \cdot \alpha\left(E_{\lambda}(\pi)\right)
$$

This will be done in the next proposition. Before stating it, we introduce some notations. If $\lambda=$ $\left(\lambda_{1}, \ldots, \lambda_{r}\right)$ is a partition of $n$, we denote by $\lambda \cdot \sigma$ the composition of $n$ defined by

$$
\lambda \cdot \sigma=\left(\lambda_{\sigma(1)}, \ldots, \lambda_{\sigma(r)}\right)
$$

We can now give the main result of this subsection.

Proposition 7.4 Let $\pi=\left(\pi_{n}\right)_{n \geq 1}$ be a family of homogeneous Lie idempotents, with $\pi_{n} \in \mathbf{S y m}_{n}$, let $\lambda=\left(l_{1}, l_{2}, \ldots, l_{r}\right)$ be a partition of $n$ and let

$$
E_{\lambda}(\pi)=\sum_{\lambda(I)=\lambda} p_{I} \pi^{I}
$$

be the idempotent associated with $\lambda$ as above. The image $\operatorname{Im} E_{\lambda}(\pi)$ of this idempotent is then the module spanned by the polynomials

$$
\sum_{\sigma \in \mathfrak{S}_{r}} p_{\lambda \cdot \sigma} P_{l_{\sigma(1)}} P_{l_{\sigma(2)}} \ldots P_{l_{\sigma(r)}}
$$

where $P_{l_{k}}$ is a homogeneous Lie polynomial of degree $l_{k}$.

Proof The proposition is a special case of the following lemma:

Lemma 7.5 With the same hypotheses, let

$$
E=\sum_{\lambda(I)=\lambda} p_{I} \pi^{I}
$$


be an arbitrary linear combination of all $\pi^{I}$ indexed by a permutation of $\lambda$. The image $\operatorname{Im} E=K\langle A\rangle_{|\lambda|}$. $\alpha(E)$ of this element is then spanned by the polynomials

$$
\sum_{\sigma \in \mathfrak{S}_{r}} p_{\lambda \cdot \sigma} P_{l_{\sigma(1)}} P_{l_{\sigma(2)}} \ldots P_{l_{\sigma(r)}}
$$

where as above, $P_{l_{k}}$ is a homogeneous Lie polynomial of degree $l_{k}$.

Proof of the lemma Let $M$ be the module defined by

$$
M=\left\{\sum_{\sigma \in \mathfrak{S}_{r}} p_{\lambda \cdot \sigma} P_{l_{\sigma(1)}} P_{l_{\sigma(2)}} \ldots P_{l_{\sigma(r)}} \mid \forall k \in[1, r], P_{l_{k}} \in L(A)_{l_{k}}\right\}
$$

We first prove that $\operatorname{Im} E \subset M$. To get this inclusion, it suffices to show that $\alpha(E) \in M$. This follows from the fact that

$$
\alpha(E)=\sum_{\substack{I=\left(i_{1}, \ldots, i_{r}\right) \\ \lambda(I)=\lambda}} p_{I} \theta_{i_{1}} \circledast \cdots \circledast \theta_{i_{r}}
$$

Going back to the definition of the convolution product, we see that

$$
\begin{aligned}
\alpha(E) & =\mu\left(\sum_{\substack{I=\left(i_{1}, \ldots, i_{r}\right) \\
\lambda(I)=\lambda}} p_{I} \vartheta_{i_{1}} \otimes \cdots \otimes \vartheta_{i_{r}}\right) \circ c(12 \ldots n) \\
& =\mu\left(\sum_{\substack{I=\left(i_{1}, \ldots, i_{r}\right) \\
\lambda(I)=\lambda}}^{\substack{\sum_{I} \\
\sum_{I=\left(i_{1}, \ldots, i_{r}\right), \lambda(I)=\lambda} \vartheta_{i_{1}} \otimes \cdots \otimes \vartheta_{i_{r}} \\
12 \ldots n \in u_{1} \amalg \ldots . . u_{r},\left|u_{k}\right|=i_{k}}} p_{I}\left(\vartheta_{i_{1}} \cdot u_{1}\right) \ldots\left(\vartheta_{i_{r}} \cdot u_{r}\right)\right. \\
& \left.=\sum_{12 \ldots n \in u_{1} \amalg \ldots u_{r}} u_{1} \otimes \otimes u_{r}\right)
\end{aligned}
$$

where $\vartheta_{i}=\alpha\left(\Psi_{i}\right)$ is Dynkin's element and where $c$ is for the usual coproduct on $K\langle A\rangle$. Observe that if $u_{1} \otimes \cdots \otimes u_{r}$ is in the support of $c(12 \ldots n)$, then $u_{\sigma(1)} \otimes \cdots \otimes u_{\sigma(r)}$ also belongs to the support of this coproduct. Taking now a set $S$ of representatives of the orbits of $r$-uples $\left(u_{1}, \ldots, u_{r}\right)$ of words under the action of $\mathfrak{S}_{r}$, we obtain

$$
\alpha(E)=\sum_{\substack{\sigma \in \mathfrak{S}_{r} \\\left(u_{1}, \ldots, u_{r}\right) \in S}} p_{\lambda \cdot \sigma}\left(\vartheta_{l_{\sigma(1)}} \cdot u_{\sigma(1)}\right) \ldots\left(\vartheta_{l_{\sigma(r)}} \cdot u_{\sigma(r)}\right)
$$


This shows that $\alpha(E) \in M$ from which it follows that $\operatorname{Im} E \subset M$, as desired.

On the other hand, a simple manipulation of the definition of the convolution product shows that

$$
\alpha(E) \cdot\left(\vartheta_{l_{1}} \ldots \vartheta_{l_{r}}\right)=\frac{1}{m_{1} ! \ldots m_{n} !}\left(\sum_{\sigma \in \mathfrak{S}_{r}} p_{\lambda \cdot \sigma} \vartheta_{l_{\sigma(1)}} \ldots \vartheta_{l_{\sigma(r)}}\right)
$$

if $\lambda=\left(1^{m_{1}}, \ldots, n^{m_{n}}\right)$, which implies that $M \subset \operatorname{Im} E$.

The following corollary is essentially a reformulation of Proposition 7.4.

Corollary 7.6 Let, for all $n \geq 1, \pi_{n}$ be a Lie idempotent of degree $n$ and set $\pi=\left(\pi_{n}\right)_{n \geq 1}$. For every partition $\lambda=\left(\lambda_{1}, \ldots, \lambda_{r}\right)$, there exists then a probability distribution $p_{\lambda}$ on $\mathfrak{S}_{r}$ with the following properties:

- $p_{\lambda}(\sigma)=p_{\lambda}(\tau)$ whenever the compositions $\lambda \cdot \sigma$ and $\lambda \cdot \tau$ are equal. This common value will be denoted by $p_{\lambda}(\sigma \cdot \lambda)$ in the sequel.

- The image of the projector $E_{\lambda}(\pi)$ is the module generated by all $p_{\lambda}$-symmetrized products

$$
\left(P_{1}, \ldots, P_{r}\right)_{p_{\lambda}}=\sum_{\sigma \in \mathfrak{S}_{r}} p_{\lambda}(\sigma \cdot \lambda) P_{\sigma(1)} \ldots P_{\sigma(r)}
$$

where each $P_{i}$ is a homogeneous Lie polynomial of degree $\lambda_{i}$.

Example 7.7 The family $\Phi=\left(\Phi_{n}\right)_{n \geq 1}$ of Solomon idempotents is characterized by the fact that the associated probability distributions are uniform.

As another simple consequence of Proposition 7.4, we can also give the structure of the multihomogeneous modules associated with Dynkin idempotents.

Corollary 7.8 Let $\lambda=\left(\lambda_{1}, \ldots, \lambda_{r}\right)$ be a partition of $n$. The image of the projector $E_{\lambda}\left(\Psi_{n}\right)$ is the module spanned by the elements

$$
\left(P_{\lambda_{1}}, \ldots, P_{\lambda_{r}}\right)_{\Psi}=\sum_{\sigma \in \mathfrak{S}_{r}} \frac{\lambda_{1} \ldots \lambda_{r}}{\lambda_{\sigma(1)}\left(\lambda_{\sigma(1)}+\lambda_{\sigma(2)}\right) \ldots\left(\lambda_{\sigma(1)}+\cdots+\lambda_{\sigma(r)}\right)} P_{\lambda_{\sigma(1)}} \ldots P_{\lambda_{\sigma(r)}}
$$

where $P_{\lambda_{i}}$ is a homogeneous Lie polynomial of degree $\lambda_{i}$.

Another consequence of Lemma 7.5, the following result is of independent interest:

Corollary 7.9 Let $\left(\pi_{n}\right)_{n \geq 1}$ be a family of Lie idempotents and let $I=\left(i_{1}^{n_{1}}, \ldots, i_{r}^{n_{r}}\right)$ be any composition of $n$ (with $i_{k} \neq i_{k+1}$ for all $k$ ). The element $\pi^{I}$ is then, up to a constant factor, an idempotent whose image under $\alpha$ is a projector onto the module

$$
(\underbrace{L(A)_{i_{1}}, \ldots, L(A)_{i_{1}}}_{n_{1} \text { terms }})(\underbrace{L(A)_{i_{2}}, \ldots, L(A)_{i_{2}}}_{n_{2} \text { terms }}) \cdots(\underbrace{L(A)_{i_{r}}, \ldots, L(A)_{i_{r}}}_{n_{r} \text { terms }})
$$

generated by the products $\left(P_{1}^{(1)}, \ldots, P_{1}^{\left(n_{1}\right)}\right) \ldots\left(P_{r}^{(1)}, \ldots, P_{r}^{\left(n_{r}\right)}\right)$ where $P_{k}^{(l)}$ is a homogeneous Lie polynomial of degree $i_{k}$ for $k \in[1, r]$ and where $(\bullet, \ldots, \bullet)$ denote the usual symmetrized product in $K\langle A\rangle$. 
Proof The only thing to check is the fact that $\pi^{I}$ is actually quasi-idempotent. This follows from Proposition 2.1 and a simple computation.

\subsection{Structure of Modules Associated with Deformations of some Classical Lie Idempotents}

We describe here without proof the structure of the multihomogeneous modules associated with the deformations of $\Phi_{n}$ and $\Psi_{n}$ defined by the transformations of alphabets $A \rightarrow(1-q) A$ and $A \rightarrow A /(1-q)$. All these results can be proved by variants of the arguments of the preceding subsection.

\subsection{1 $\Phi_{n}((1-q) A)$}

We need first to introduce a $q$-analogue of the factorial, connected with the combinatorics of descents and compositions.

Let $r \geq 1$ be an integer and let $S$ be a subset of $[1, r]$. We associate with $S$ the vector $v(S)=$ $\left(v(S)_{1}, \ldots, v(S)_{r}\right) \in\{0,1\}^{r}$ where $v(S)_{i}=1$ iff $i \in S$. We can then consider a new vector $s(S)=$ $\left(s(S)_{1}, \ldots, s(S)_{r}\right) \in \mathbf{N}^{r}$ defined by

$$
s(S)_{i}=v(S)_{i}+\cdots+v(S)_{r}
$$

for $i \in[1, r]$.

Example 7.10 Table 2 gives the values of the above vectors and numbers for all subsets of $[1,1],[1,2]$ and $[1,3]$.

\begin{tabular}{|c||c||c|c|c|}
\hline$C(S)$ & $S$ & $v(S)$ & $s(S)$ & $d_{S}$ \\
\hline \hline 2 & $\emptyset$ & $(0)$ & $(0)$ & 1 \\
11 & $\emptyset$ & $(1)$ & $(1)$ & 1 \\
\hline \hline 3 & $\emptyset$ & $(0,0)$ & $(0,0)$ & 1 \\
21 & $\{2\}$ & $(0,1)$ & $(1,1)$ & 2 \\
12 & $\{1\}$ & $(1,0)$ & $(1,0)$ & 2 \\
111 & $\{1,2\}$ & $(1,1)$ & $(2,1)$ & 1 \\
\hline \hline 4 & $\emptyset$ & $(0,0,0)$ & $(0,0,0)$ & 1 \\
31 & $\{3\}$ & $(0,0,1)$ & $(1,0,0)$ & 3 \\
22 & $\{2\}$ & $(0,1,0)$ & $(1,1,0)$ & 5 \\
211 & $\{2,3\}$ & $(0,1,1)$ & $(2,2,1)$ & 3 \\
13 & $\{1\}$ & $(1,0,0)$ & $(1,0,0)$ & 3 \\
121 & $\{1,3\}$ & $(1,0,1)$ & $(2,1,1)$ & 5 \\
112 & $\{1,2\}$ & $(1,1,0)$ & $(2,1,0)$ & 3 \\
1111 & $\{1,2,3\}$ & $(1,1,1)$ & $(3,2,1)$ & 1 \\
\hline
\end{tabular}


Let now $I=\left(i_{1}, \ldots, i_{r}\right)$ be a composition of length $r$. We associate with $I$ the $q$-analogue of $(r+1)$ ! defined by

$$
[(r+1) !]_{I, q}=\sum_{S \subset[1, r]} d_{S} q^{s(S)_{1} i_{1}+\cdots+s(S)_{r} i_{r}}
$$

where $d_{S}=\left|\left\{\sigma \in \mathfrak{S}_{r+1}, D(\sigma)=S\right\}\right|$ denotes the number of permutations of $\mathfrak{S}_{r+1}$ whose descent set is equal to $S$.

Example 7.11 For $r=1, r=2$ and $r=3$, one can read on the table the following formulas:

$$
\begin{gathered}
{[2 !]_{\left(i_{1}\right), q}=1+q^{i_{1}}} \\
{[3 !]_{\left(i_{1}, i_{2}\right), q}=1+2 q^{i_{1}+i_{2}}+2 q^{i_{1}}+q^{2 i_{1}+i_{2}}} \\
{[4 !]_{\left(i_{1}, i_{2}, i_{3}\right), q}=1+3 q^{i_{1}+i_{2}+i_{3}}+5 q^{i_{1}+i_{2}}+3 q^{2 i_{1}+2 i_{2}+i_{3}}+3 q^{i_{1}}+5 q^{2 i_{1}+i_{2}+i_{3}}+3 q^{2 i_{1}+i_{2}}+q^{3 i_{1}+2 i_{2}+i_{3}}}
\end{gathered}
$$

For a composition $I=\left(i_{1}, \ldots, i_{r}\right)$ of length $r$, set

$$
a_{I}(q)=\frac{[r !]_{\left(i_{1}, \ldots, i_{r-1}\right)}, q}{r !} \frac{\left[i_{1}\right]_{q}\left[i_{2}\right]_{q} \ldots\left[i_{r}\right]_{q}}{\left[i_{1}\right]_{q}\left[i_{1}+i_{2}\right]_{q} \ldots\left[i_{1}+\cdots+i_{r}\right]_{q}}
$$

Let now $\lambda=\left(\lambda_{1}, \ldots, \lambda_{r}\right)$ be a partition of $n$. We can now define a $q$-deformation of the usual symmetrized product by

$$
\left(x_{\lambda_{1}}, \ldots, x_{\lambda_{r}}\right)_{\Phi((1-q) A)}=\sum_{\sigma \in \mathfrak{S}_{r}} a_{\sigma \cdot \lambda}(q) x_{\lambda_{\sigma(1)}} \ldots x_{\lambda_{\sigma(r)}}
$$

This product clearly reduces to the ordinary symmetrized product for $q=0$. Also, the family $a=$ $\left(a_{\sigma \lambda \lambda}(q)\right)_{\sigma \in \mathfrak{S}_{r}}$ is a probability distribution over $\mathfrak{S}_{r}$, as we will see in the sequel. The following result provides therefore a complete description, of the multihomogeneous modules associated with the Lie idempotents $\Phi_{n}((1-q) A) /\left(n\left(1-q^{n}\right)\right)$.

Proposition 7.12 Let $\lambda=\left(\lambda_{1}, \ldots, \lambda_{r}\right)$ be a partition of $n$. Then the image of the projector $E_{\lambda}\left(\Phi_{n}((1-\right.$ q) $\left.A) /\left(1-q^{n}\right)\right)$ is the module generated by all products of the form

$$
\left(P_{\lambda_{1}}, \ldots, P_{\lambda_{r}}\right)_{\Phi((1-q) A)}
$$

where $P_{\lambda_{i}}$ is an arbitrary homogeneous Lie polynomial of order $\lambda_{i}$.

Note that the case $q=1$ gives again Corollary 7.8. 


\subsection{2 $\Phi_{n}(A /(1-q))$}

For a composition $I=\left(i_{1}, \ldots, i_{r}\right)$, define now

$$
B_{I}(q)=\sum_{k=1}^{r}\left(\begin{array}{l}
r-1 \\
k-1
\end{array}\right)(-1)^{k-1} q^{i_{1}+\cdots+i_{k-1}}\left[i_{k}\right]_{q},
$$

and set

$$
b_{I}(q)=\frac{B_{I}(q)}{(1-q)^{r-1} r !\left[i_{1}\right]_{q} \ldots\left[i_{r}\right]_{q}}
$$

The relevant $q$-deformation of the symmetrized product is defined by

$$
\left(x_{\lambda_{1}}, \ldots, x_{\lambda_{r}}\right)_{\Phi(A /(1-q))}=\sum_{\sigma \in \mathfrak{S}_{r}} b_{\sigma \lambda \lambda} x_{\lambda_{\sigma(1)}} \ldots x_{\lambda_{\sigma(r)}}
$$

Indeed, we have:

Proposition 7.13 Let $\lambda=\left(\lambda_{1}, \ldots, \lambda_{r}\right)$ be a partition of $n$. Then the image of the projector $E_{\lambda}\left(\Phi_{n}(A /(1-\right.$ $\left.q)) /\left(1-q^{n}\right)\right)$ is the module generated by the products

$$
\left(P_{\lambda_{1}}, \ldots, P_{\lambda_{r}}\right)_{\Phi(A /(1-q))}
$$

where $P_{\lambda_{i}}$ is an arbitrary homogeneous Lie polynomial of degree $\lambda_{i}$.

7.3.3 $\Psi_{n}((1-q) A)$

Let $I=\left(i_{1}, \ldots, i_{r}, i_{r+1}\right)$ be a composition of length $r+1$. We associate with it the family $\left(\gamma_{S, I}\right)_{S \subset[1, r]}$ of $\mathbf{N}^{2^{r}}$ defined by $\gamma_{\emptyset,\left(i_{1}\right)}=1$ for every $i_{1} \geq 1$ (when $r=0$ ) and by the inductive rules

$$
\forall S \subset[1, r-1],\left\{\begin{array}{c}
\gamma_{S \cup\{r\}, I}=\left(\begin{array}{c}
i_{r-1}+i_{r}-1 \\
i_{r}
\end{array}\right) \gamma_{S,\left(i_{1}, \ldots, i_{r-2}, i_{r-1}+i_{r}\right)}, \\
\gamma_{S, I}=\left(\begin{array}{c}
i_{1}+\cdots+i_{r} \\
i_{r}
\end{array}\right) \gamma_{S,\left(i_{1}, \ldots, i_{r-1}\right)}-\left(\begin{array}{c}
i_{r-1}+i_{r}-1 \\
i_{r}
\end{array}\right) \gamma_{S,\left(i_{1}, \ldots, i_{r-2}, i_{r-1}+i_{r}\right)}
\end{array}\right.
$$

(when $r \geq 1$ ). Next, we introduce the polynomial $C_{I}(q) \in \mathbf{N}[q]$ defined by

$$
C_{I}(q)=\sum_{S \subset[1, r]} \gamma_{S, I} q^{s(S)_{1} i_{1}+\cdots+s(S)_{r} i_{r}}
$$

Example 7.14 Let us show how to compute $C_{(2,3,1)}(q)$. We need first to compute

$$
\begin{gathered}
\gamma_{\{1\},(2,3)}=\left(\begin{array}{l}
4 \\
3
\end{array}\right)=4, \gamma_{\emptyset,(2,3)}=\left(\begin{array}{l}
5 \\
3
\end{array}\right)-\left(\begin{array}{l}
4 \\
3
\end{array}\right)=10-4=6, \\
\gamma_{\{1\},(2,4)}=\left(\begin{array}{l}
5 \\
4
\end{array}\right)=5, \gamma_{\emptyset,(2,4)}=\left(\begin{array}{l}
6 \\
4
\end{array}\right)-\left(\begin{array}{l}
5 \\
4
\end{array}\right)=15-5=10 .
\end{gathered}
$$


It follows that

$$
\begin{gathered}
\gamma_{\{1,2\},(2,3,1)}=\left(\begin{array}{l}
3 \\
1
\end{array}\right) \gamma_{\{1\},(2,4)}=3 \times 5=15, \gamma_{\{2\},(2,3,1)}=\left(\begin{array}{l}
3 \\
1
\end{array}\right) \gamma_{\emptyset,(2,4)}=3 \times 10=30, \\
\gamma_{\{1\},(2,3,1)}=\left(\begin{array}{l}
6 \\
1
\end{array}\right) \gamma_{\{1\},(2,3)}-\gamma_{\{1,2\},(2,3,1)}=6 \times 4-15=9, \\
\gamma_{\emptyset,(2,3,1)}=\left(\begin{array}{l}
6 \\
1
\end{array}\right) \gamma_{\emptyset,(2,3)}-\gamma_{\{2\},(2,3,1)}=6 \times 6-30=6 .
\end{gathered}
$$

Hence we finally get

$$
\begin{aligned}
C_{(2,3,1)}(q) & =15 q^{2 \times 2+1 \times 3}+30 q^{1 \times 2+1 \times 3}+9 q^{1 \times 2+0 \times 3}+6 q^{0 \times 2+0 \times 3} \\
& =15 q^{7}+30 q^{5}+9 q^{2}+6
\end{aligned}
$$

For a composition $I=\left(i_{1}, \ldots, i_{r}\right)$ of length $r$, let us set

$$
c_{I}(q)=\frac{\left[i_{1}\right]_{q} \ldots\left[i_{r}\right]_{q}}{\left[i_{1}\right]_{q}\left[i_{1}+i_{2}\right]_{q} \ldots\left[i_{1}+\cdots+i_{r}\right]_{q}} \frac{C_{I}(q)}{\left(\begin{array}{c}
i_{1}+\cdots+i_{r} \\
i_{1}, \ldots, i_{r}
\end{array}\right)}
$$

Let $\lambda=\left(\lambda_{1}, \ldots, \lambda_{r}\right)$ be a partition of $n$. The $q$ - symmetrized product will be in this case

$$
\left(x_{1}, \ldots, x_{n}\right)_{\Psi((1-q) A)}=\sum_{\sigma \in \mathfrak{S}_{r}} c_{\sigma \cdot \lambda}(q) x_{\lambda_{\sigma(1)}} \ldots x_{\lambda_{\sigma(r)}}
$$

and we have:

Proposition 7.15 Let $\lambda=\left(\lambda_{1}, \ldots, \lambda_{r}\right)$ be a partition of $n$. Then the image of the projector $E_{\lambda}\left(\Psi_{n}(A /(1-\right.$ $\left.q)) /\left(1-q^{n}\right)\right)$ is the module generated by all products of the form

$$
\left(P_{\lambda_{1}}, \ldots, P_{\lambda_{r}}\right) \Psi((1-q) A)
$$

where $P_{\lambda_{i}}$ is an arbitrary homogeneous Lie polynomial of degree $\lambda_{i}$.

7.3.4 $\Psi_{n}(A /(1-q))$

For $I=\left(i_{1}, \ldots, i_{r}\right)$, define the polynomial $D_{I}(q)$ by the recurrence relations

$$
\left\{\begin{aligned}
D_{i_{1}} & =\left[i_{1}\right]_{q} \\
D_{I}(q) & =\sum_{k=1}^{r} D_{i_{1}, \ldots, i_{k-1}, i_{k}-1, i_{k+1}, \ldots, i_{r}}(q)+\left(\begin{array}{c}
|I|-1 \\
i_{1}-1
\end{array}\right) q^{i_{1}-1} D_{i_{2}, \ldots, i_{r}}(q) \quad \text { for } l(I) \geq 2
\end{aligned}\right.
$$

with the convention that $D \ldots, 0, \ldots(q)=0$. We set

$$
d_{I}(q)=\frac{D_{I}(q)}{\left(\begin{array}{c}
|I| \\
i_{1}, \ldots, i_{r}
\end{array}\right)\left[i_{1}\right]_{q} \ldots\left[i_{r}\right]_{q}}
$$


The $q$-analogue of the symmetrized product will be defined by

$$
\left(x_{\lambda_{1}}, \ldots, x_{\lambda_{r}}\right)_{\Psi(A /(1-q))}=\sum_{\sigma \in \mathcal{G}_{r}} d_{\sigma \cdot \lambda} x_{\lambda_{\sigma(1)}} \ldots x_{\lambda_{\sigma(r)}}
$$

Proposition 7.16 Let $\lambda=\left(\lambda_{1}, \ldots, \lambda_{r}\right)$ be a partition of $n$. Then the image of the projector $E_{\lambda}\left(\Psi_{n}(A /(1-\right.$ $\left.q)) /\left(1-q^{n}\right)\right)$ is the module generated by all products of the form

$$
\left(P_{\lambda_{1}}, \ldots, P_{\lambda_{r}}\right) \Psi(A /(1-q))
$$

where $P_{\lambda_{i}}$ is an arbitrary homogeneous Lie polynomial of degree $\lambda_{i}$.

\section{Aknowledgements}

The authors are grateful to R. Stanley for communicating Varchenko's reference [13], to D. Zagier for explaining his work [25], to R. Speicher for providing references [12] and [17], and to D. Svrtan for his remarks.

\section{References}

[1] Garsia, A. and Reutenauer, C. (1989). A decomposition of Solomon's descent algebra. Adv. in Math. 77, 189-262.

[2] Reutenauer, C. (1994). Free Lie Algebras. Oxford University Press.

[3] Mielnik , B. and Plebański, J. (1970). Combinatorial approach to Baker-Campbell-Hausdorff exponents. Ann. Inst. Henri Poincaré, Section A XII, 215-254.

[4] Gerstenhaber, M. and Schack, D. (1987). A Hodge-type decomposition for commutative algebra cohomology. J. Pure Appl. Alg. 48, 229-247.

[5] Loday, J. L. (1989). Opérations sur l'homologie cyclique des algèbres commutatives. Invent. Math. 96, 205-230.

[6] Solomon, L. (1976). A Mackey formula in the group ring of a Coxeter group. J. Algebra 41, 255-268.

[7] Krob, D., Leclerc, B. and Thibon, J.-Y. (1997). Noncommutative symmetric functions II: Transformations of alphabets, Int. J. Alg. Comp. 7, 181-264.

[8] Duchamp, G., Krob, D., Leclerc, B. and Thibon, J.-Y. (1994). Déformations de projecteurs de Lie. C.R. Acad. Sci. Paris, 319, 909-914.

[9] Duchamp, G. (1991). Orthogonal projection onto the free Lie algebra. Theor. Comput. Sci. 79, 227-239.

[10] Ree, R. (1960). Generalized Lie elements. Canad. J. Math. 12, 493-502. 
[11] Greenberg, O. W. (1991). Particles with small violations of Fermi or Bose statistics. Physical Rev. D 43, 4111-4120.

[12] Bożejko, M. and Speicher, R. (1991). An example of a generalized brownian motion. Commun. Math. Phys. 137, 519-531.

[13] Varchenko, A. (1993). Bilinear form of real configuration of hyperplanes. Adv. in Maths. 97, 110144.

[14] Norton, P. N. (1979). 0-Hecke algebras. J. Austral. Math. Soc. (Series A) 27, 337-357.

[15] Duchamp, G., Krob, D., Leclerc, B. and Thibon, J.-Y. (1996). Fonctions quasi-symétriques, fonctions symétriques non-commutatives, et algèbres de Hecke à $q=0$. C.R. Acad. Sci. Paris 322, $107-112$.

[16] Kashiwara, M. and Miwa, T. and Stern, E. (1996). Decomposition of $q$-deformed Fock spaces. Selecta Math. 1, 787-801.

[17] Meljanac, S. and Svrtan, D. (1996). Study of Gram matrices in Fock representation of multiparametric canonical commutation relations, extended Zagier's conjecture, hyperplane arrangements and quantum groups. Math. Comm. 1, 1-24.

[18] Gelfand, I. M., Krob, D., Lascoux, A., Leclerc, B., Retakh, V. S. and Thibon, J.-Y. (1995). Noncommutative symmetric functions. Adv. in Math., 112, 218-348.

[19] Gelfand, I. M. and Retakh, V. S. (1991). Determinants of matrices over noncommutative rings. Funct. Anal. Appl. 25, 91-102.

[20] Gelfand, I. M. and Retakh, V. S. (1992). A theory of noncommutative determinants and characteristic functions of graphs. Funct. Anal. Appl. 26, 1-20.

[21] Macdonald, I. G. (1979). Symmetric Functions and Hall Polynomials. Oxford University Press (2nd ed. 1995).

[22] Malvenuto, C. and Reutenauer, C. (1993). Duality between quasi-symmetric functions and the Solomon descent algebra. Preprint UQAM, Montréal.

[23] Gessel, I. (1984). Multipartite P-partitions and inner product of skew Schur functions. Contemp. Math. 34, 289-301.

[24] Rosso, M. (1995). Groupes quantiques et algèbres de battage quantiques. C.R. Acad. Sci. Paris Ser. I 320, 145-148.

[25] Zagier, D. (1992). Realizability of a model in infinite statistics. Commun. Math. Phys. 147, 199210.

[26] Fivel, D. I. (1990). Interpolation between Bose and Fermi statistics using generalized commutators. Phys. Rev. Lett. 65, 3361-3364.

[27] Fivel, D. I. (1992). Errata. Phys. Rev. Lett. 65, 2020. 
[28] Woronowicz, S. L. (1989). Differential calculus on compact matrix pseudogroups (Quantum groups). Commun. Math. Phys. 122, 125-170.

[29] Nichols, W. (1991). Bialgebras of type I. Commun. Alg. 19, 3061-3073.

[30] Rosso, M. (1996). Personal communication.

[31] Duchamp, G., Krob, D., Lascoux, A., Leclerc, B., Scharf, T. and Thibon, J.-Y. (1995). EulerPoincaré characteristic and polynomial representations of Iwahori-Hecke algebras. Publications of the R.I.M.S., Kyoto University, 31, 179-201.

[32] Stern, E. (1995). Semi-infinite wedges and vertex operators. Int. Math. Res. Notices 4, 210-220.

[33] Berstel, J. and Reutenauer, C. (1988). Rational series and their languages. EATCS Monographs on Theoretical Computer Science, Springer-Verlag.

[34] Schützenberger, M. P. (1958). Sur une propriété combinatoire des algèbres de Lie libres pouvant être utilisée dans un problème de mathématiques appliquées, Sém. P. Dubreil, Faculté des Sciences, Paris.

[35] Kawski, M. (1994). Chronological algebras and non-linear control. Asian Control Conference, Tokyo.

[36] Schechtman, V. and Varchenko, A. (1991). Quantum groups and homology of local systems. 'Algebraic geometry and anal. geometry', ICM-90 Satellite Conference, pp. 182-191. SpringerVerlag.

[37] Leclerc, B., Scharf, T. and Thibon, J.-Y. (1996). Noncommutative cyclic characters of symmetric groups. J. Comb. Theory A 75, 55-69. 